\title{
On the regularity of the interface of a thermodynamically consistent two-phase Stefan problem with surface tension
}

\author{
JAN PRÜSS \\ Institut für Mathematik, Martin-Luther-Universität Halle-Wittenberg, 60120 Halle, Germany \\ E-mail: jan.pruess@mathematik.uni-halle.de \\ YUANZHEN SHAO \\ Department of Mathematics, Vanderbilt University, Nashville, TN 37240, USA \\ E-mail: yuanzhen.shao@vanderbilt.edu \\ GIERI SIMONETT \\ Department of Mathematics, Vanderbilt University, Nashville, TN 37240, USA \\ E-mail: gieri.simonett@vanderbilt.edu
}

[Received 16 December 2014 and in revised form 28 September 2015]

\begin{abstract}
We study the regularity of the free boundary arising in a thermodynamically consistent two-phase Stefan problem with surface tension by means of a family of parameter-dependent diffeomorphisms, $L_{p}$-maximal regularity theory, and the implicit function theorem.
\end{abstract}

2010 Mathematics Subject Classification: Primary 35R35, 82C26, 35K55, 35B65

Keywords: Free boundary problems; phase transitions; the Stefan problem; regularity of moving interfaces; real analytic solutions; maximal regularity; the implicit function theorem.

\section{Introduction}

The main objective of this article is to develop a technique relying on a family of parameterdependent diffeomorphisms, maximal regularity theory, and the implicit function theorem to prove regularity of moving interfaces occurring in free boundary problems. As an application, we prove that the moving interface in a thermodynamically consistent two-phase Stefan problem is jointly $C^{k}$-smooth in time and space, for $k \in \mathbb{N} \cup\{\infty, \omega\}$ with $\omega$ being the symbol of real analyticity, as long as several physical quantities, that is, the coefficients of the heat conductivity, kinetic undercooling, and the free energy, enjoy appropriate regularity assumptions.

The idea of establishing regularity of solutions to differential equations by means of the implicit function theorem in conjunction with a translation argument was first introduced by S.B. Angenent in [8] to prove analyticity of the free boundary in one dimensional porous medium equations, and has proved itself a useful tool later in many publications. See for example [9, 27, 28, 50, 58]. More precisely, to study the regularity of the solution to a differential equation, one introduces parameters representing translation in space and time into the solution to the given differential equation. Then one studies the parameter-dependent equation satisfied by this transformed solution. The implicit function theorem yields the smooth dependence upon the parameters of the solution to the parameter-dependent problem. This regularity property is then inherited by the original solution. An advantage of this technique is reflected by its power to prove analyticity of solutions to differential equations, which cannot be attained through the classical method of bootstrapping. 
A well-known approach to free boundary problems is to transform the original problem with a moving boundary, or separating interface, which we denote by $\Gamma(t)$, into one with a fixed reference manifold $\Sigma$ by means of the Hanzawa transformation, see [39]. Then the problem of establishing the regularity of the free boundary $\Gamma(t)$ is transferred into establishing the regularity of the height function parameterizing $\Gamma(t)$ over $\Sigma$. However, applying the aforementioned translation technique to the height function on the surface $\Sigma$ causes an essential challenge, considering for instance the usual translation $(t, x) \mapsto(t+\lambda, x+\mu)$, because of the global nature of these translations. Hence we desire an alternative that only shifts the variables "locally". The idea of localizing translations was first introduced by J. Escher, J. Prüss and G. Simonett in [26] to study regularity of solutions to elliptic and parabolic equations in Euclidean space. The basic building block of [26] consists of rescaling translations by some cutoff function. This technique was later generalized in Y. Shao [73] to introduce a family of parameter-dependent diffeomorphisms acting on functions or tensor fields on Riemannian manifolds by means of a smooth atlas in order to study the regularity of solutions to geometric evolution equations. But in view of the physical quantities in the bulk phases adjacent to $\Gamma(t)$, e.g., the temperature function in the case of phase transitions, or the velocity and the pressure field in the case of two-phase fluids, we need introduce a localized translation not only on the fixed reference surface $\Sigma$, but also in a neighborhood of $\Sigma$. This adds one more degree of complexity to the aforementioned technique for geometric flows. In Section 3, we will build up a complete theory of parameter-dependent diffeomorphisms for free boundary problems.

Free boundary problems form an important field of applied analysis. They deal with solving partial differential equations in a given domain, a part of whose boundary is a priori unknown. That portion of the boundary is called the free boundary or the moving boundary. In addition to the standard boundary conditions that are needed in order to solve the prescribed partial differential equations, an additional condition must be imposed at the free boundary. One then seeks to determine both the free boundary and the solution of the differential equation. This field has drawn great attention over decades due to its applications to physics, chemistry, medicine, material science, and so forth.

Great progress has been seen in the studies of regularity of free boundaries during the past half century. Many mathematicians have made contributions to the theory of free boundary problems, among them H.W. Alt, H. Berestycki, L.A. Caffarelli, A. Friedman, D. Kinderlehrer, L. Nirenberg, J. Spruck, G. Stampacchia [2, 3, 12, 14, 16, 43-46], E. DiBenedetto [24]. We also refer the reader to [33, 46, 67-69] for a historical account of the field.

The Stefan problem, arguably, is the most studied free boundary problem, with over 1200 mathematical publications devoted to the topic. It was first introduced in 1889 by J. Stefan. We refer the reader to the books [53, 71, 77] for further information. The Stefan problem describes phase transitions in liquid-solid systems and accounts for heat diffusion and exchange of latent heat in a homogeneous medium, wherein the liquid and solid phases are separated by a closed moving interface $\Gamma(t)$. The basic physical law governing this process is conservation of energy. To be more precise, let $\Omega \subset \mathbb{R}^{m+1}$ be a bounded domain of class $C^{2}, m \geqslant 1$. $\Omega$ is occupied by a material that can undergo phase changes: at time $t$, phase $i$ occupies the subdomain $\Omega_{i}(t)$ of $\Omega$, respectively, with $i=1,2$. We assume that $\partial \Omega_{1}(t) \cap \partial \Omega=\emptyset$; this means that no boundary contact can occur. The closed compact hypersurface $\Gamma(t):=\partial \Omega_{1}(t) \subset \Omega$ forms the interface between the phases. Then the Stefan problem with surface tension, or the Stefan problem with Gibbs-Thomson correction, can 
be formulated as follows:

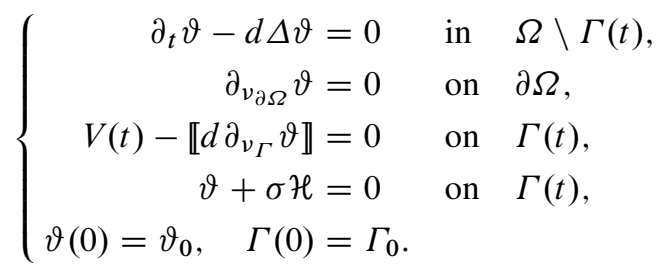

Here $\vartheta(t)=\vartheta_{1}(t) \chi_{\Omega_{1}(t)}+\vartheta_{2}(t) \chi \Omega_{2}(t)$, where $\vartheta_{i}$ denotes the relative temperature distribution in phase $i, v_{\Gamma}(t)$ the outer normal field of $\partial \Omega_{1}(t), V(t)$ the normal velocity of $\Gamma(t), \mathcal{H}(t)=$ $\mathcal{H}(\Gamma(t))=-\operatorname{div}_{\Gamma(t)} v_{\Gamma}(t) / m$ the mean curvature of $\Gamma(t)$, and $\llbracket v \rrbracket=\left.v_{2}\right|_{\Gamma(t)}-\left.v_{1}\right|_{\Gamma(t)}$ the jump of a quantity $v$ across $\Gamma(t)$. The sign of the mean curvature $\mathcal{H}$ is chosen to be negative at a point $x \in \Gamma$ if $\Omega_{1} \cap \mathbb{B}(x, r)$ is convex for some sufficiently small $r>0$. Thus if $\Omega_{1}$ is a ball of radius $R$ then $\mathcal{H}=-1 / R$ for its boundary $\Gamma$. The function $d$ agrees with a positive constant $d_{i}$ in $\Omega_{i}(t)$, which stand for the heat conductivities in different phases. The condition that $V(t)=\llbracket d \partial_{\nu} \vartheta \rrbracket$ is caused by the law of conservation of energy and is usually called the Stefan condition.

If a pair $(\vartheta, \Gamma)$ solves the system of equations (1.1), $\Gamma$ is called the free boundary to the Stefan problem. In the case that the condition $\vartheta+\sigma \mathcal{H}=0$ is replaced by

$$
\vartheta=0 \quad \text { on } \quad \Gamma(t)
$$

i.e., if $\sigma=0$, the resulting problem is usually referred to as the classical Stefan problem. Here we mention the monographs by B. Chalmers [20, Chapter 1], P. Hartman [40], A. Visintin [77], and the research papers by G. Caginalp [19], M. E. Gurtin [34-36], J. S. Langer [48], W. W. Mullins and R. F. Sekerka [56, 57], A. Visintin [76], where this law has been motivated and derived based on various mathematical and physical principles. As has been explained in [65, Section 1], see also [64], condition (1.2) can be understood as a first order approximation of the relation

$$
\llbracket \psi(\theta) \rrbracket+\sigma \mathcal{H}=0 \quad \text { on } \quad \Gamma(t)
$$

around the melting temperature, where $\theta$ is the absolute temperature and $\psi$ is the free energy of the system.

In the absence of one of $\vartheta_{i}$, that is, one of $\vartheta_{i}$ is identically equal to some constant, the problem is called the one-phase Stefan problem, otherwise it is alluded to as the two-phase Stefan problem.

In the one-dimensional case, different aspects of the classical Stefan problem were extensively studied before the 1980s. In higher dimensions, global existence and uniqueness of weak solutions was first established by S.L. Kamenomostskaja in [42], see also A. Friedman [30]. Existence of local classical solutions was obtained by E.I. Hanzawa [39] and A.M. Měrmanov [52], provided that the initial data are smooth enough and satisfy some higher order compatibility conditions.

In the case of the one-phase classical Stefan problem, regularity of the free boundary for weak solutions is studied in $[14,15,32,43,44]$. The formulation of the problem as a parabolic variational inequality was initiated by G. Duvaut in [25]. After that, it has been applied in [32] to get the Lipschitz continuity of the free boundary under some conditions on the initial geometry and the given data. Later in a paper of L.A. Caffarelli [14], the author proved that the free boundary is $C^{1}$ in space and time and the temperature is $C^{2}$ up to the free boundary near a density point for the states set, namely the solid phase. In particular, if the free boundary is Lipschitz continuous 
then it is actually $C^{1}$. In [15], the author identified conditions to guarantee a locally Lipschitz free boundary. Almost at the same time, D. Kinderlehrer and L. Nirenberg [43] established via the partial hodograph and Legendre transformations in conjunction with a variational inequality argument smoothness of the free boundary as well as the solution. The conditions in [43] can be verified by the results in [14]. In a subsequent paper [44], the same authors showed under some integral conditions by the same technique that the free boundary and the solution are analytic in the space variables and in the second Gevrey class for the time variable. Regularization of the free boundary for large time was obtained by H. Matano in [51], i.e., any weak solution eventually becomes smooth. Continuity of the temperature distribution for weak solutions is proved in [18].

Regularity of the free boundary for weak solutions or viscosity solutions has been explored in $[10,11,47]$ for the multi-dimensional two-phase Stefan problem based on a non-degeneracy condition. The non-degeneracy condition states, roughly speaking, that the heat fluxes are not vanishing simultaneously on the free boundary, which is automatically satisfied when the free boundary is regular enough, say belongs to the Hölder class of order more than 1. Assuming that the free boundary is locally Lipschitz continuous, it was proven in [10] that the solution is classical, i.e., the free boundary and the viscosity solution are $C^{1}$. Later the authors showed that the non-degeneracy condition can be replaced by some flatness condition. In the presence of the nondegeneracy condition, $\mathrm{H}$. Koch verified that the $C^{1}$ free boundary and the temperature are in fact smooth. Through a different approach by imposing a mild regularity condition on the initial data, it was shown by J. Prüss, J. Saal and G. Simonett in [58] that the free boundary and the temperature are analytic under the assumption that the free boundary can be expressed as the graph of some function. Continuity of the temperature distribution for weak solutions was obtained in [17, 23, 24, 78].

Although the Stefan problem with the Gibbs-Thomson correction (1.2) has been around for many decades, only few analytical results concerning existence of solutions can be found in the literature. A. Friedman and F. Reitich [31] considered the case with small surface tension $0<\sigma \ll 1$ and linearized the problem about $\sigma=0$. Assuming the existence of a smooth solution for the case $\sigma=0$, that is, for the classical Stefan problem, the authors proved existence and uniqueness of a weak solution for the linearized problem and then investigated the effect of small surface tension on the shape of $\Gamma(t)$. Existence of long time weak solutions was first established by Luckhaus [49], using a discretized problem and a capacity-type estimate for approximating solutions. The weak solutions obtained have a sharp interface, but are highly non-unique. See also Röger [70], and Almgren and Wang [1]. Existence of classical solutions, but without uniqueness, was proved by E.V. Radkevich in [66]. In A.M. Meirmanov [54], the way in which a spherical ball of ice in a supercooled fluid melts down was investigated. It was presented by J. Escher, J. Prüss and G. Simonett in [27] that there exists a unique local strong solution and the free boundary immediately regularizes to be analytic in space and time provided that the initial data satisfy some mild regularity assumptions. This result is based on the assumption that the free boundary is given by the graph of some function. In J. Prüss and G. Simonett [59], linearized stability and instability of equilibria was investigated. Nonlinear stability results were obtained by M. Hadžić, Y. Guo [37, 38] and J. Prüss, G. Simonett and R. Zacher [65], see also the monograph [64].

In this paper we consider a general model for phase transitions, formulated in [65], that is thermodynamically consistent, see [7] and [41] for related work. It involves the thermodynamic quantities of absolute temperature, free energy, internal energy, and entropy, and is complemented by constitutive equations for the free energies and the heat fluxes in the bulk regions. An important 
assumption is that there be no entropy production on the interface. In particular, the interface is assumed to carry no mass and no energy except for surface tension.

To be more precise, we choose $\Omega, \Omega_{i}(t)$ and $\Gamma(t)$ as in (1.1). By the thermodynamically consistent two-phase Stefan problem with surface tension, possibly with kinetic undercooling, we mean the following problem: find a family of closed compact hypersurfaces $\{\Gamma(t)\}_{t \geqslant 0}$ contained in $\Omega$ and an appropriately smooth function $\theta: \mathbb{R}_{+} \times \bar{\Omega} \rightarrow \mathbb{R}$ such that

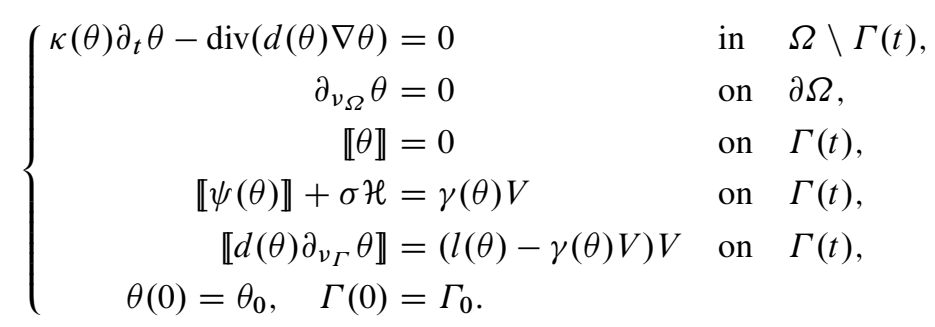

Here $\theta$ denotes the (absolute) temperature. Several quantities are derived from the free energies $\psi_{i}(\theta)$ as follows:

- $\epsilon_{i}(\theta)=\psi_{i}(\theta)+\theta \eta_{i}(\theta)$, the internal energy in phase $i$,

- $\eta_{i}(\theta)=-\psi_{i}^{\prime}(\theta)$, the entropy,

- $\kappa_{i}(\theta)=\epsilon_{i}^{\prime}(\theta)=-\theta \psi_{i}^{\prime \prime}(\theta)>0$, the heat capacity,

- $l(\theta)=\theta \llbracket \psi^{\prime}(\theta) \rrbracket=-\theta \llbracket \eta(\theta) \rrbracket$, the latent heat.

Furthermore, $d_{i}(\theta)>0$ denotes the coefficient of heat conduction in Fourier's law, $\gamma(\theta) \geqslant 0$ the coefficient of kinetic undercooling. As is commonly done, we assume that there exists a unique (constant) melting temperature $\theta_{m}$, characterized by the equation $\llbracket \psi\left(\theta_{m}\right) \rrbracket=0$. Finally, system (1.4) is to be completed by constitutive equations for the free energies $\psi_{i}$ in the bulk phases $\Omega_{i}(t)$.

In the sequel we drop the index $i$, as there is no danger of confusion; we just keep in mind that the coefficients depend on the phases. The temperature is assumed to be continuous across the interface, as indicated by the condition $\llbracket \theta \rrbracket=0$ in (1.4). However, the free energy and the heat conductivities depend on the respective phases, and hence the jumps $\psi(\theta) \rrbracket, \llbracket \kappa(\theta) \rrbracket, \llbracket \eta(\theta) \rrbracket, \llbracket d(\theta) \rrbracket$ are in general non-zero at the interface. We assume that the coefficient of surface tension is constant.

In this paper, we will prove the following regularity result for $k \in \mathbb{N} \cup\{\infty, \omega\}$.

Theorem $1.1(\gamma \equiv 0)$. Let $p>m+3, \gamma=0, \sigma>0$. Suppose that $d_{i} \in C^{k+2}(0, \infty)$, $\psi_{i} \in C^{k+3}(0, \infty)$ for $i=1,2$ such that

$$
\kappa_{i}(u)=-u \psi_{i}^{\prime \prime}(u)>0, \quad d_{i}(u)>0, \quad u \in(0, \infty) .
$$

Assume the regularity conditions

$$
\theta_{0} \in W_{p}^{2-2 / p}\left(\Omega \backslash \Gamma_{0}\right) \cap C(\bar{\Omega}), \quad \theta_{0}>0, \quad \Gamma_{0} \in W_{p}^{4-3 / p},
$$

the compatibility conditions

$$
\partial_{\nu_{\Omega}} \theta_{0}=0, \quad \llbracket \psi\left(\theta_{0}\right) \rrbracket+\sigma \mathcal{H}\left(\Gamma_{0}\right)=0, \quad \llbracket d\left(\theta_{0}\right) \partial_{\nu_{\Gamma_{0}}} \theta_{0} \rrbracket \in W_{p}^{2-6 / p}\left(\Gamma_{0}\right),
$$

and the well-posedness condition

$$
l\left(\theta_{0}\right) \neq 0 \quad \text { on } \Gamma_{0} .
$$


Then there exists a unique $L_{p}$-solution $(u, \Gamma)$ for the Stefan problem with surface tension (1.4) on some possibly small but nontrivial time interval $J=[0, T]$, and

$$
m:=\bigcup_{t \in(0, T)}\{\{t\} \times \Gamma(t)\}
$$

is a $C^{k}$-manifold in $\mathbb{R}^{m+2}$. In particular, each manifold $\Gamma(t)$ is $C^{k}$ for $t \in(0, T)$.

The result in the presence of kinetic undercooling reads as

TheOREM $1.2(\gamma>0)$. Let $p>m+3, \sigma>0$. Suppose that $d_{i}, \gamma \in C^{k+2}(0, \infty), \psi_{i} \in$ $C^{k+3}(0, \infty)$ for $i=1,2$ such that

$$
\kappa_{i}(u)=-u \psi_{i}^{\prime \prime}(u)>0, \quad d_{i}(u)>0, \quad \gamma(u)>0, \quad u \in(0, \infty) .
$$

Assume the regularity conditions

$$
\theta_{0} \in W_{p}^{2-2 / p}\left(\Omega \backslash \Gamma_{0}\right) \cap C(\bar{\Omega}), \quad \theta_{0}>0, \quad \Gamma_{0} \in W_{p}^{4-3 / p},
$$

and the compatibility conditions

$$
\partial_{\nu_{\Omega}} \theta_{0}=0, \quad\left(\llbracket \psi\left(\theta_{0}\right) \rrbracket+\sigma \mathcal{H}\left(\Gamma_{0}\right)\right)\left(l\left(\theta_{0}\right)-\llbracket \psi\left(\theta_{0}\right) \rrbracket-\sigma \mathcal{H}\left(\Gamma_{0}\right)\right)=\gamma\left(\theta_{0}\right) \llbracket d\left(\theta_{0}\right)_{\Gamma_{0}} \theta_{0} \rrbracket .
$$

Then there exists a unique $L_{p}$-solution $(\theta, \Gamma)$ for the Stefan problem with surface tension (1.4) on some possibly small but nontrivial time interval $J=[0, T]$, and

$$
m:=\bigcup_{t \in(0, T)}\{\{t\} \times \Gamma(t)\}
$$

is a $C^{k}$-manifold in $\mathbb{R}^{m+2}$. In particular, each manifold $\Gamma(t)$ is $C^{k}$ for $t \in(0, T)$.

Remark 1.3. (a) For $k \in \mathbb{N} \cup\{\infty\}$, under the conditions in Theorems 1.1 and 1.2, we can show that the temperature satisfies

$$
\theta \in C^{k}(((0, T) \times \Omega) \backslash m)
$$

See Remark 4.5 for a justification. However, in the case $k=\omega$, additional work is needed to establish the interior analyticity of $\theta$ in the bulk phases. In order to keep this already long paper at a reasonable length, we will refrain from establishing this here.

(b) According to [65, Theorem 3.9] we obtain a solution of (1.4) in the state manifold $\delta m$ on a maximal interval of existence $\left[0, t_{*}\right)$. The regularity assertions of Theorem 1.1 and Theorem 1.2, respectively, then hold true on $\left[0, t_{*}\right)$.

Notation. Throughout this paper, we always assume that

- $E$ denotes a finite dimensional Banach space.

- $m+3<p<\infty$, and $s \geqslant 0$, unless stated otherwise.

Given two Banach spaces $X, Y$, the notation $\mathcal{L}(X, Y)$ stands for the set of all bounded linear operators from $X$ to $Y$, and $\mathcal{L}$ is $(X, Y)$ denotes the set of all bounded linear isomorphisms from $X$ to $Y$.

For any topological sets $U$ and $V, U \subset \subset V$ means that $\bar{U} \subset \stackrel{\circ}{V}$ with $\bar{U}$ compact. 


\section{Parameterization over a fixed interface}

Let $\Omega \subset \mathbb{R}^{m+1}$ be a bounded domain with boundary $\partial \Omega$ of class $C^{2}$, and suppose that $\Gamma_{0} \subset \Omega$ is a closed embedded hypersurface of class $C^{2}$, that is, a $C^{2}$-manifold which is the boundary of a bounded domain $\Omega_{1}(0) \subset \Omega$. Set $\Omega_{2}(0):=\Omega \backslash \bar{\Omega}_{1}(0)$. Following $[63,64]$, we may approximate $\Gamma_{0}$ by an $m$-dimensional real analytic compact closed oriented reference hypersurface $(\Sigma, g)$, with $g$ the tangential metric induced by the Euclidean metric $g_{m+1}$, in the sense that there exists a function $h_{0} \in C^{2}(\Sigma,(-a, a))$ such that the map

$$
\Lambda_{h_{0}}: \Sigma \rightarrow \mathbb{R}^{m+1}: \quad\left[\mathrm{p} \mapsto \mathrm{p}+h_{0}(\mathrm{p}) \nu_{\Sigma}(\mathrm{p})\right]
$$

is a diffeomorphism from $\Sigma$ onto $\Gamma_{0}$, where $v_{\Sigma}$ is the outer normal of $\Sigma$. The positive constant $a$ depends on the inner and outer ball conditions of $\Sigma$. It is well-known that $\Sigma$ admits a $a$-tubular neighborhood $\mathrm{T}_{a}$, which means that the map

$$
\Lambda: \Sigma \times(-a, a) \rightarrow \mathbb{R}^{m+1}:(\mathrm{p}, r) \mapsto \mathrm{p}+r v_{\Sigma}(\mathrm{p})
$$

is a diffeomorphism from the fiber bundle $\Sigma \times(-a, a)$ onto $\operatorname{im}(\Lambda):=\mathrm{T}_{a}$. For sufficiently small $a$, $\overline{\mathrm{T}}_{a} \subset \Omega$. $\Sigma$ bounds a domain $\Omega_{1}$, and we set $\Omega_{2}=\Omega \backslash \bar{\Omega}_{1}$. The inverse $\Lambda^{-1}$ can be decomposed as

$$
\Lambda^{-1}: \mathrm{T}_{a} \rightarrow \Sigma \times(-a, a): \quad z \mapsto\left(\Pi(z), d_{\Sigma}(z)\right)
$$

where $\Pi(z)$ is the metric projection of $z$ onto $\Sigma$ and $d_{\Sigma}(z)$ denotes the signed distance from $z$ to $\Sigma$ such that $\left|d_{\Sigma}(z)\right|=\operatorname{dist}(z, \Sigma)$ and $d_{\Sigma}(z)<0$ iff $z \in \Omega_{1}$. It follows from the inverse function theorem that the maps $\Pi$ and $d_{\Sigma}$ are both real analytic.

We may use the map $\Lambda$ to parameterize the unknown free boundary $\Gamma(t)$ over $\Sigma$ by a height function $h(t): \Sigma \rightarrow \mathbb{R}$ via

$$
\Gamma(t):=\left\{\mathrm{p}+h(t, \mathrm{p}) v_{\Sigma}(\mathrm{p}), \quad \mathrm{p} \in \Sigma\right\}, \quad t \geqslant 0,
$$

for small $t \geqslant 0$, at least. $\Gamma(t)$ bounds a bounded region $\Omega_{1}(t)$. Put $\Omega_{2}(t)=\Omega \backslash \bar{\Omega}_{1}(t)$. Choosing an auxiliary function $\zeta \in \Phi((-2 a / 3,2 a / 3),[0,1])$ such that $\left.\zeta\right|_{(-a / 3, a / 3)} \equiv 1$, we may extend the above diffeomorphism onto $\bar{\Omega}$ via

$$
\Xi_{h}(t, z)=z+\zeta\left(d_{\Sigma}(z)\right) h(t, \Pi(z)) v_{\Sigma}(\Pi(z))=: z+\Upsilon(h)(t, z) .
$$

We have transformed the time varying regions $\Omega_{i}(t)$ to the fixed domains $\Omega_{i}$. This is the direct mapping method, also known as Hanazawa transformation. By means of this transformation, we obtain the following transformed problem for $\vartheta(t, z):=\theta\left(t, \Xi_{h}(t, z)\right)$ :

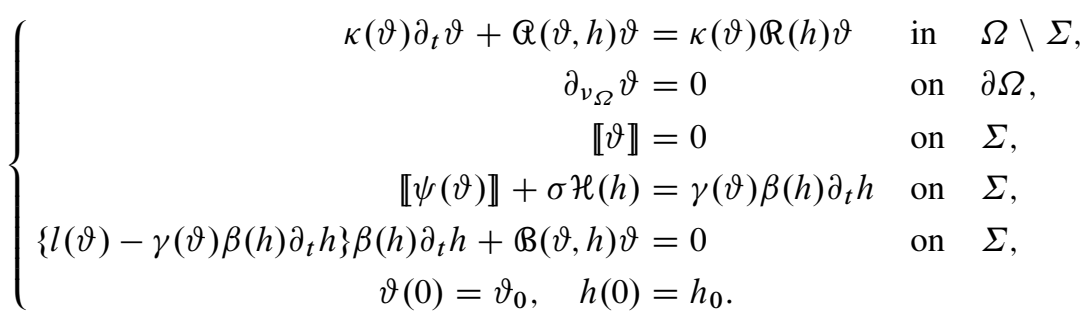


Here $Q(\vartheta, h)$ and $B(\vartheta, h)$ denote the transformations of $-\operatorname{div}(d \nabla)$ and $-\llbracket d \partial_{v_{\Gamma}} \rrbracket$, respectively. Moreover, $\mathcal{H}(h)$ stands for the mean curvature of the hypersurface

$$
\Gamma_{h}:=\Lambda_{h}(\Sigma)
$$

The term $\beta(h) \partial_{t} h$ represents the normal velocity $V$ with $\beta(h):=\left(v_{\Sigma} \mid v_{\Gamma}(h)\right)$, where $v_{\Gamma}(h)$ is the outer normal field of $\Gamma_{h}$, and

$$
R(h) \vartheta:=\partial_{t} \vartheta-\partial_{t} \theta \circ \Xi_{h} .
$$

It is shown in [65] that $v_{\Gamma}(h)=\beta(h)\left(v_{\Sigma}-\alpha(h)\right)$, and

$$
\beta(h)=\left(1+|\alpha(h)|^{2}\right)^{-1 / 2}, \quad \mathbb{R}(h) \vartheta=\left(\nabla \vartheta \mid\left[I+\nabla \Upsilon(h)^{\top}\right]^{-1} \partial_{t} \Upsilon(h)\right)
$$

Here with the Weingarten map $L_{\Sigma}=-\nabla_{\Sigma} v_{\Sigma}$, we have

$$
\alpha(h):=M_{0}(h) \nabla_{\Sigma} h, \quad \text { where } \quad M_{0}(h):=\left(I-h L_{\Sigma}\right)^{-1} .
$$

With $\partial_{v}:=\partial_{\nu_{\Sigma}}$, the operator $\mathbb{B}(\vartheta, h)$ becomes

$$
\begin{aligned}
\mathbb{B}(\vartheta, h) \vartheta & =-\llbracket d(\theta) \partial_{\nu_{\Gamma}} \theta \rrbracket \circ \Xi_{h}=-\left(\llbracket d(\vartheta)\left(I-M_{1}(h)\right) \nabla \vartheta \rrbracket \mid v_{\Gamma}\right) \\
& =-\beta(h)\left(\llbracket d(\vartheta)\left(I-M_{1}(h)\right) \nabla \vartheta \rrbracket \mid v_{\Sigma}-\alpha(h)\right) \\
& =-\beta(h) \llbracket d(\vartheta) \partial_{\nu} \vartheta \rrbracket+\beta(h)\left(\llbracket d(\vartheta) \nabla \vartheta \rrbracket \mid\left(I-M_{1}(h)\right)^{\top} \alpha(h)\right),
\end{aligned}
$$

where $M_{1}(h):=\left[\left(I+\nabla \Upsilon(h)^{\top}\right)^{-1} \nabla \Upsilon(h)^{\top}\right]^{\top}$, and finally

$$
\begin{aligned}
Q(\vartheta, h) \vartheta= & -\operatorname{div}(d(\theta) \nabla \theta) \circ \Xi_{h}=-\left(\left(I-M_{1}(h)\right) \nabla \mid d(\vartheta)\left(I-M_{1}(h)\right) \nabla \vartheta\right) \\
= & -d(\vartheta) \Delta \vartheta+d(\vartheta)\left[M_{1}(h)+M_{1}^{\top}(h)-M_{1}(h) M_{1}^{\top}(h)\right]: \nabla^{2} \vartheta \\
& -d^{\prime}(\vartheta)\left|\left(I-M_{1}(h)\right) \nabla \vartheta\right|^{2}+d(\vartheta)\left(\left(I-M_{1}(h)\right): \nabla M_{1}(h) \mid \nabla \vartheta\right) .
\end{aligned}
$$

We recall that, for matrices $A, B \in \mathbb{R}^{n \times n}, A: B=\sum_{i, j=1}^{n} a_{i j} b_{i j}=\operatorname{tr}\left(A B^{\top}\right)$ denotes the inner product.

We set

$$
\begin{aligned}
\mathbb{E}_{1}(J) & :=\left\{\vartheta \in H_{p}^{1}\left(J ; L_{p}(\Omega)\right) \cap L_{p}\left(J ; H_{p}^{2}(\Omega \backslash \Sigma)\right): \llbracket \vartheta \rrbracket=0, \partial_{\nu_{\Omega}} \vartheta=0\right\}, \\
\mathbb{E}_{2}(J) & := \begin{cases}W_{p}^{3 / 2-1 / 2 p}\left(J ; L_{p}(\Sigma)\right) \cap W_{p}^{1-1 / 2 p}\left(J ; H_{p}^{2}(\Sigma)\right) \cap L_{p}\left(J ; W_{p}^{4-1 / p}(\Sigma)\right), \quad \gamma \equiv 0, \\
W_{p}^{2-1 / 2 p}\left(J ; L_{p}(\Sigma)\right) \cap L_{p}\left(J ; W_{p}^{4-1 / p}(\Sigma)\right), \quad \gamma>0,\end{cases} \\
\mathbb{E}(J) & :=\mathbb{E}_{1}(J) \times \mathbb{E}_{2}(J),
\end{aligned}
$$

that is, $\mathbb{E}(J)$ denotes the solution space for (2.1). Similarly, we define

$$
\left\{\begin{aligned}
\mathbb{F}_{1}(J) & :=L_{p}\left(J ; L_{p}(\Omega)\right) \\
\mathbb{F}_{2}(J) & :=W_{p}^{1-1 / 2 p}\left(J ; L_{p}(\Sigma)\right) \cap L_{p}\left(J ; W_{p}^{2-1 / p}(\Sigma)\right), \\
\mathbb{F}_{3}(J) & :=W_{p}^{1 / 2-1 / 2 p}\left(J ; L_{p}(\Sigma)\right) \cap L_{p}\left(J ; W_{p}^{1-1 / p}(\Sigma)\right), \\
\mathbb{F}_{4} & :=\left[W_{p}^{2-2 / p}(\Omega \backslash \Sigma) \cap C(\bar{\Omega})\right] \times W_{p}^{4-3 / p}(\Sigma) .
\end{aligned}\right.
$$


A left subscript zero means vanishing time trace at $t=0$, whenever it exists. So for example ${ }_{0} \mathbb{E}_{2}(J)=\left\{h \in \mathbb{E}_{2}(J): h(0)=\partial_{t} h(0)=0\right\}$ for $p>3$.

Whenever $(J)$ is replaced by $(J, U)$, or $(J, U ; E)$ for any set $U$, e.g., in $\mathbb{E}(J)$, it always means that in the corresponding spaces the original spatial domain is replaced by $U$ and the functions become $E$-valued in the latter case. For example,

$$
\mathbb{F}_{2}\left(J, \mathrm{~T}_{a} ; E\right):=W_{p}^{1-1 / 2 p}\left(J ; L_{p}\left(\mathrm{~T}_{a}, E\right)\right) \cap L_{p}\left(J ; W_{p}^{2-1 / p}\left(\mathrm{~T}_{a}, E\right)\right) .
$$

We equip $\Sigma$ with a normalized real analytic atlas $\mathfrak{A}=\left(\mathrm{O}_{\kappa}, \varphi_{\kappa}\right)_{\kappa \in \mathfrak{K}}$, where $\mathfrak{K}$ is a finite index set, in the sense that $\varphi_{\kappa}\left(\mathrm{O}_{\kappa}\right)=\mathrm{Q}^{m}:=(-1,1)^{m}$, for every $\kappa$. Let $\psi_{\kappa}:=\left[\varphi_{\kappa}\right]^{-1}$. Then we may endow $\Omega$ with a real analytic atlas $\mathfrak{A}_{\mathfrak{e}}=\left(\mathrm{O}_{\mathfrak{e}, \kappa}, \varphi_{\mathfrak{e}, \kappa}\right)_{\kappa \in \mathfrak{K}_{\mathfrak{e}}}$ with $\mathfrak{K}_{\mathfrak{e}}:=\mathfrak{K} \cup\left\{\kappa_{1}, \kappa_{2}\right\}$ by the following construction. When $\kappa \in \mathfrak{K}$

$$
\mathrm{O}_{\mathfrak{e}, \kappa}:=\mathrm{T}_{a, \kappa}:=\Lambda\left(\mathrm{O}_{\kappa} \times(-a, a)\right), \quad \varphi_{\mathfrak{e}, \kappa}(z):=\left(\varphi_{\kappa} \circ \Pi(z), d_{\Sigma}(z)\right), \quad z \in \mathrm{O}_{\mathfrak{e}, \kappa} .
$$

Let $\mathrm{Q}_{a}:=\mathrm{Q}^{m} \times(-a, a)$ and

$$
\psi_{\mathfrak{e}, \kappa}:=\left[\varphi_{\mathfrak{e}, \kappa}\right]^{-1}: \mathrm{Q}_{a} \rightarrow \mathrm{O}_{\mathfrak{e}, \kappa}: \quad\left[(x, y) \mapsto \psi_{\kappa}(x)+y v_{\Sigma}\left(\psi_{\kappa}(x)\right)\right]
$$

with $x \in \mathrm{Q}^{m}$ and $y \in(-a, a)$. For $\kappa \notin \mathfrak{K}$, we set

$$
\left(\mathrm{O}_{\mathfrak{e}, \kappa_{i}}, \varphi_{\mathfrak{e}, \kappa_{i}}\right):=\left(\mathrm{O}_{\mathfrak{e}, \kappa_{i}}, \operatorname{id}_{\mathrm{O}_{\mathfrak{e}, \kappa_{i}}}\right), \quad i=1,2,
$$

where $\mathrm{O}_{\mathfrak{e}, \kappa_{i}}:=\Omega_{i} \backslash \overline{\mathrm{T}}_{a / 6}$ for $i=1,2$. It is immediate from our construction that the atlas $\mathfrak{A}_{\mathfrak{e}}$ is real analytically compatible with the Euclidean structure of $\Omega$. Moreover, all the transition maps are $C^{\infty}$-continuous and have bounded derivatives, which we refer to as $B C^{\infty}$-continuous.

A family $\left(\pi_{\kappa}\right)_{\kappa \in \mathfrak{K}}$ is called a localization system subordinate to $\mathfrak{A}$ if

$$
\pi_{\kappa} \in \Phi\left(\mathrm{O}_{\kappa},[0,1]\right) \text { and }\left(\pi_{\kappa}^{2}\right)_{\kappa \in \mathfrak{K}} \text { is a partition of unity subordinate to }\left(\mathrm{O}_{\kappa}\right)_{\kappa \in \mathfrak{K}} \text {. }
$$

Based on [5, Lemma 3.2], $\Sigma$ admits a localization system $\left(\pi_{\kappa}\right)_{\kappa \in \mathfrak{K}}$ subordinate to $\mathfrak{A}$. Using the cutoff function $\zeta$ introduced above, we may construct a new localization system $\left(\pi_{\mathfrak{e}, \kappa}\right)_{\kappa \in \mathfrak{K}_{\mathfrak{e}}}$ subordinate to $\mathfrak{A}_{\mathfrak{e}}$ as follows:

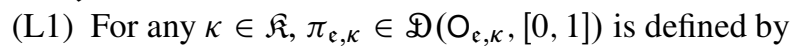

$$
\pi_{\mathfrak{e}, \kappa}(z):= \begin{cases}\zeta\left(d_{\Sigma}(z)\right) \pi_{\kappa}(\Pi(z)), & z \in \mathrm{O}_{\mathfrak{e}, \kappa}, \\ 0, & z \notin \mathrm{O}_{\mathfrak{e}, \kappa} .\end{cases}
$$

(L2) For $i=1,2, \pi_{\mathfrak{e}, \kappa_{i}} \in C^{\infty}\left(\overline{\mathrm{O}}_{\mathfrak{c}_{i}, \kappa_{i}},[0,1]\right)$ is defined by

$$
\pi_{\mathfrak{e}, \kappa_{i}}(z)= \begin{cases}\frac{1-\zeta\left(d_{\Sigma}(z)\right)}{\sqrt{\left(1-\zeta\left(d_{\Sigma}(z)\right)\right)^{2}+\zeta^{2}\left(d_{\Sigma}(z)\right)},} & z \in \Omega_{i} \cap \mathrm{T}_{a}, \\ 1, & z \in \bar{\Omega}_{i} \backslash \mathrm{T}_{a} .\end{cases}
$$

$\left(\pi_{\mathfrak{e}, \kappa}^{2}\right)_{\kappa \in \mathfrak{K}_{\mathfrak{e}}}$ forms a localization system subordinate to $\left(\mathrm{O}_{\mathfrak{e}, \kappa}\right)_{\kappa \in \mathfrak{K}_{\mathfrak{e}}}$. We put

$$
\mathbb{X}_{\mathfrak{e}, \kappa}:= \begin{cases}\mathbb{R}^{m+1}, & \kappa \in \mathfrak{K}, \\ \mathrm{O}_{\mathfrak{e}, \kappa_{i}}, & i=1,2 .\end{cases}
$$


For any finite dimensional Banach space $E$, the maps $\mathbb{R}_{\mathfrak{e}, \kappa}^{c}$ and $\mathbb{R}_{\mathfrak{e}, \kappa}$ are defined by

$$
\mathbb{R}_{\mathfrak{e}, \kappa}^{c}: L_{1, l o c}(\Omega, E) \rightarrow L_{1, l o c}\left(\mathbb{X}_{\mathfrak{e}, \kappa}, E\right): u \mapsto \psi_{\mathfrak{e}, \kappa}^{*} \pi_{\mathfrak{e}, \kappa} u, \quad \kappa \in \mathfrak{K}_{\mathfrak{e}},
$$

and

$$
\mathbb{R}_{\mathfrak{e}, \kappa}: L_{1, l o c}\left(\mathbb{X}_{\mathfrak{e}, \kappa}, E\right) \rightarrow L_{1, l o c}(\Omega, E): u \mapsto \pi_{\mathfrak{e}, \kappa} \varphi_{\mathfrak{e}, \kappa}^{*} u, \quad \kappa \in \mathfrak{K}_{\mathfrak{e}}
$$

Here and in the following it is understood that a partially defined and compactly supported map is automatically extended over the whole base manifold by identifying it to be zero outside its original domain. Moreover, let

$$
\mathbb{R}_{\mathfrak{e}}^{c}: L_{1, l o c}(\Omega, E) \rightarrow \prod_{\kappa \in \mathfrak{K}_{\mathfrak{e}}} L_{1, l o c}\left(\mathbb{X}_{\mathfrak{e}, \kappa}, E\right): u \mapsto\left(\mathbb{R}_{\mathfrak{e}, \kappa}^{c} u\right)_{\kappa \in \mathfrak{K}_{\mathfrak{e}}}
$$

and

$$
\mathbb{R}_{\mathfrak{e}}: \prod_{\kappa \in \mathfrak{K}_{\mathfrak{e}}} L_{1, l o c}\left(\mathbb{X}_{\mathfrak{e}, \kappa}, E\right) \rightarrow L_{1, l o c}(\Omega, E):\left(u_{\kappa}\right)_{\kappa} \mapsto \sum_{\kappa \in \mathfrak{K}_{\mathfrak{e}}} \pi_{\mathfrak{e}, \kappa} \varphi_{\mathfrak{e}, \kappa}^{*} u
$$

On the manifold $\Sigma$, similar maps $\mathbb{R}_{\kappa}^{c}, \mathbb{R}^{c}, \mathbb{R}_{\kappa}$, and $\mathbb{R}$ are defined in terms of $\pi_{\kappa}, \varphi_{\kappa}$, and $\psi_{\kappa}$. See [73].

\section{$2.1 \quad$ Function spaces}

For any open subset $U \subset \mathbb{R}^{n}$, the Banach space $B C^{k}(U, E)$ is defined by

$$
B C^{k}(U, E):=\left(\left\{u \in C^{k}(U, E):\|u\|_{k, \infty}<\infty\right\},\|\cdot\|_{k, \infty}\right) .
$$

The closed linear subspace $B U C^{k}(U, E)$ of $B C^{k}(U, E)$ consists of all functions $u \in B C^{k}(U, E)$ such that $\partial^{\alpha} u$ is uniformly continuous for all $|\alpha| \leqslant k$. Moreover,

$$
B C^{\infty}(U, E):=\bigcap_{k} B C^{k}(U, E)=\bigcap_{k} B U C^{k}(U, E) .
$$

It is a Fréchet space equipped with the natural projective topology.

For $0<s<1,0<\delta \leqslant \infty$ and $u \in E^{U}$, the seminorm $[\cdot]_{s, \infty}^{\delta}$ is defined by

$$
[u]_{s, \infty}^{\delta}:=\sup _{h \in(0, \delta)^{n}} \frac{\|u(\cdot+h)-u(\cdot)\|_{\infty}}{|h|^{s}}, \quad[\cdot]_{s, \infty}:=[\cdot]_{s, \infty}^{\infty} .
$$

Let $k<s<k+1$. The Hölder space $B C^{s}(U, E)$ is defined as

$$
B C^{s}(U, E):=\left(\left\{u \in B C^{k}(U, E):\|u\|_{s, \infty}<\infty\right\},\|\cdot\|_{s, \infty}\right),
$$

where $\|u\|_{s, \infty}:=\|u\|_{k, \infty}+\max _{|\alpha|=k}\left[\partial^{\alpha} u\right]_{s-k, \infty}$.

In order to have a general theory that is applicable to situations other than the Stefan problem, we also introduce the little Hölder space of order $s \geqslant 0$, which is defined by

$$
b c^{s}(U, E):=\text { the closure of } B C^{\infty}(U, E) \text { in } B C^{s}(U, E) .
$$


By [6, formula (11.13), Corollary 11.2, Theorem 11.3], we have

$$
b c^{k}(U, E)=B U C^{k}(U, E),
$$

and for $k<s<k+1$

$$
u \in B C^{s}(U, E) \text { belongs to } b c^{s}(U, E) \text { iff } \lim _{\delta \rightarrow 0}\left[\partial^{\alpha} u\right]_{s-[s], \infty}^{\delta}=0, \quad|\alpha|=[s] .
$$

The spaces $\mathfrak{F}^{s}(\Sigma, E)$ with $\mathfrak{F} \in\left\{b c, B C, W_{p}, H_{p}\right\}$ are defined in terms of the smooth atlas $\mathfrak{A}$, that is, $u \in \mathfrak{F}^{s}(\Sigma, E)$ iff $\psi_{\kappa}^{*} u:=u \circ \psi_{\kappa} \in \mathfrak{F}^{s}\left(\mathrm{Q}^{m}, E\right)$ for all $\kappa \in \mathfrak{K}$. See [5] and [73].

Throughout, for any finite index set $\mathbb{A}$ and Banach spaces $E_{\alpha}, \alpha \in \mathbb{A}$, it is understood that the space $\mathbb{E}:=\prod_{\alpha \in \mathbb{A}} E_{\alpha}$ is equipped with the maximum norm. For $\mathfrak{F} \in\left\{b c, B C, B U C, W_{p}, H_{p}\right\}$, we put

$$
\mathfrak{F}_{\mathfrak{e}}^{s}:=\prod_{\kappa \in \mathfrak{K}_{\mathfrak{e}}} \mathfrak{F}^{s}\left(\mathbb{X}_{\mathfrak{e}, \kappa}, E\right), \quad \mathfrak{F}^{s}:=\prod_{\kappa \in \mathfrak{K}} \mathfrak{F}_{\kappa}^{s},
$$

where $\mathfrak{F}_{\kappa}^{s}=\mathfrak{F}^{s}\left(\mathbb{R}^{m}, E\right)$.

Proposition 2.1 Suppose that $\mathfrak{B}=\mathfrak{F}$ when $\mathfrak{F} \in\left\{b c, W_{p}, H_{p}\right\}$, or $\mathfrak{B} \in\{B C, B U C\}$ when $\mathfrak{F}=B C$. Then

(a) $\mathbb{R}$ is a retraction from $\mathfrak{B}^{s}$ onto $\mathfrak{F}^{s}(\Sigma, E)$ with $\mathbb{R}^{c}$ as a coretraction. Moreover, the case $s<0$ and $\mathfrak{F} \in\left\{W_{p}, H_{p}\right\}$ is also admissible.

(b) $\mathbb{R}_{\mathfrak{e}}$ is a retraction from $\mathfrak{B}_{\mathfrak{e}}^{s}$ onto $\mathfrak{F}^{s}(\Omega, E)$ with $\mathbb{R}_{\mathfrak{e}}^{c}$ as a coretraction.

Proof. (a) The assertions are special cases of [6, Theorem 9.3] and [73, Propositions 2.1, 2.2].

(b) For $\kappa \in \mathfrak{K}$, the boundedness of the maps $\mathbb{R}_{\mathfrak{e}, \kappa}$ and $\mathbb{R}_{\mathfrak{e}, \kappa}^{c}$ follows from the proofs in [5, Section 6 , 7] and [6, Section 12]. The continuity of the maps $\mathbb{R}_{\mathfrak{e}, \kappa_{i}}^{c}$ and $\mathbb{R}_{\mathfrak{e}, \kappa_{i}}$ is straightforward.

Let $J:=[0, T]$ for some $T>0$. Due to the temporal independence of the above retractioncoretraction systems, we readily infer that

Proposition 2.2 Let $r \geqslant 0$. Suppose that $\mathfrak{C} \in\left\{C, W_{p}\right\}$, and $\mathfrak{B}=\mathfrak{F}$ when $\mathfrak{F} \in\left\{b c, W_{p}, H_{p}\right\}$, or $\mathfrak{B} \in\{B C, B U C\}$ when $\mathfrak{F}=B C$. Then

(a) $\mathbb{R}$ is a retraction from $\mathfrak{C}^{r}\left(J ; \mathfrak{B}^{s}\right)$ onto $\mathfrak{C}^{r}\left(J ; \mathfrak{F}^{s}(\Sigma, E)\right)$ with $\mathbb{R}^{c}$ as a coretraction. Moreover, the case $s<0$ and $\mathfrak{F} \in\left\{W_{p}, H_{p}\right\}$ is also admissible.

(b) $\mathbb{R}_{\mathfrak{e}}$ is a retraction from $\mathfrak{C}^{r}\left(J ; \mathfrak{B}_{\mathfrak{e}}^{s}\right)$ onto $\mathfrak{C}^{r}\left(J ; \mathfrak{F}^{s}(\Omega, E)\right)$ with $\mathbb{R}_{\mathfrak{e}}^{c}$ as a coretraction.

\section{Parameter-dependent diffeomorphisms}

3.1 Model diffeomorphisms in Euclidean space

Given $z_{c} \in \mathrm{T}_{a / 3, \kappa_{c}} \backslash \Sigma \subset \Omega$ for some $\kappa_{c} \in \mathfrak{K}$, let

$$
\left(x_{c}, y_{c}\right)=\left(\varphi_{\kappa_{c}} \circ \Pi\left(z_{c}\right), d_{\Sigma}\left(z_{c}\right)\right) .
$$

We may choose a sufficiently small positive constant $\varepsilon_{0}$ such that

$$
\mathbb{B}^{m}\left(x_{c}, 5 \varepsilon_{0}\right) \subset \mathrm{Q}^{m}, \quad \mathbb{B}\left(y_{c}, 5 \varepsilon_{0}\right) \subset(-1)^{i}(0, a / 3) .
$$

Let $\mathbb{B}_{\varepsilon, \alpha}:=\mathbb{B}^{m}\left(x_{c}, \varepsilon\right) \times(-\alpha, \alpha)$. We pick several auxiliary functions in the following manner: 
- $\varpi \in \Phi(-13 a / 18,13 a / 18),[0,1])$, and $\left.\varpi\right|_{(-2 a / 3,2 a / 3)} \equiv 1$.

- $\chi_{m} \in \Phi\left(\mathbb{B}^{m}\left(x_{c}, 2 \varepsilon_{0}\right),[0,1]\right)$, and $\left.\chi_{m}\right|_{\mathbb{B}^{m}\left(x_{c}, \varepsilon_{0}\right)} \equiv 1$.

- $\chi \in \Phi\left(\mathbb{B}\left(y_{c}, 2 \varepsilon_{0}\right),[0,1]\right)$, and $\left.\chi\right|_{\mathbb{B}\left(y_{c}, \varepsilon_{0}\right)} \equiv 1$.

- $\zeta_{\mathfrak{e}} \in \boxplus\left(\psi_{\kappa_{c}}\left(\mathbb{B}_{5 \varepsilon_{0}, 17 a / 18}\right),[0,1]\right)$, and $\left.\zeta_{\mathfrak{e}}\right|_{\psi_{\kappa_{c}}\left(\mathbb{B}_{4 \varepsilon_{0}, 8 a / 9}\right)} \equiv 1$. We set $\zeta:=\left.\zeta_{\mathfrak{e}}\right|_{\Sigma}$

Then we can introduce a localized translation on $Q_{a}$ as follows

$$
\theta_{\mu, \eta}:(x, y) \mapsto\left(x+\chi_{m}(x) \varpi(y) \mu, y+\chi_{m}(x) \chi(y) \eta\right), \quad(\mu, \eta) \in \mathbb{B}\left(0, r_{0}\right),
$$

with $\mu \in \mathbb{R}^{m}, \eta \in \mathbb{R}$ and sufficiently small $r_{0}>0$. Henceforth, $\mathbb{B}\left(x_{0}, r_{0}\right)$ always denotes the ball with radius $r_{0}$ centered at $x_{0}$ in $\mathbb{R}^{n}$. The dimension $n$ of the ball is not distinguished as long as it is clear from the context.

The related partial translations in horizontal/vertical directions can be defined separately as

$$
\left\{\begin{array}{l}
\theta_{\mu}:(x, y) \mapsto\left(x+\chi_{m}(x) \varpi(y) \mu, y\right), \\
\bar{\theta}_{\eta}:(x, y) \mapsto\left(x, y+\chi_{m}(x) \chi(y) \eta\right) .
\end{array}\right.
$$

For $r_{0}$ small and every $(\mu, \eta) \in \mathbb{B}\left(0, r_{0}\right)$

$$
\theta_{\mu, \eta}=\theta_{\mu} \circ \bar{\theta}_{\eta}
$$

It is not hard to show that for sufficiently small $r_{0}$, we have $\theta_{\mu, \eta}\left(\mathbb{B}_{3 \varepsilon_{0}, 7 a / 9}\right) \subset \mathbb{B}_{3 \varepsilon_{0}, 7 a / 9}$ for any $(\mu, \eta) \in \mathbb{B}\left(0, r_{0}\right)$. (3.1) implies that

$$
\theta_{\mu, \eta}^{*}=\bar{\theta}_{\eta}^{*} \circ \theta_{\mu}^{*}, \quad \text { and } \quad \theta_{*}^{\mu, \eta}:=\left[\theta_{\mu, \eta}\right]_{*}=\theta_{*}^{\mu} \circ \bar{\theta}_{*}^{\eta} .
$$

Since $\theta_{\mu}$ and $\bar{\theta}_{\eta}$ are the restrictions of truncated translations in the sense of those defined in [26] in horizontal and vertical direction, respectively, the readers should have no difficulty convincing themselves that all the results in [26] are at our disposal for functions defined on an open subset $U \subset \mathbb{R}^{m+1}$ and the transformations $\theta_{\mu}^{*}$ and $\bar{\theta}_{\eta}^{*}$. Taking advantage of these considerations, we will state some properties of $\theta_{\mu, \eta}^{*}$ for later use in the following with brief proofs.

Hereafter, we assume $O \subset U \subset \mathbb{R}^{m+1}$ are open, and $O$ contains $\overline{\mathbb{B}}_{3 \varepsilon_{0}, 7 a / 9}$. We conclude from $\theta_{\mu, \eta}(U) \subset U$ that $\theta_{\mu, \eta}^{*}: \mathfrak{F}^{S}(U, E) \rightarrow E^{U}$. In the rest of this section, we always assume the following, unless mentioned otherwise,

$$
1<p<\infty, \quad n, l \in \mathbb{N}_{0}, \quad k \in \mathbb{N}_{0} \cup\{\infty, \omega\} .
$$

Proposition 3.1 Suppose that $\mathfrak{F} \in\left\{b c, B U C, W_{p}, H_{p}\right\}$ and $u \in C^{n+k}(O, E) \cap \mathfrak{F}^{s}(U, E)$ with $s \in[0, n]$ when $\mathfrak{F} \in\left\{b c, W_{p}, H_{p}\right\}$, or with $s=n$ when $\mathfrak{F}=B U C$. Then for sufficiently small $r_{0}$, (a) $\theta_{\mu, \eta} \in \operatorname{Diff}^{\infty}(U),(\mu, \eta) \in \mathbb{B}\left(0, r_{0}\right)$.

(b) $\theta_{\mu, \eta}^{*} \in \operatorname{Lis}\left(\mathfrak{F}^{s}(U, E)\right),\left[\theta_{\mu, \eta}^{*}\right]^{-1}=\theta_{*}^{\mu, \eta},(\mu, \eta) \in \mathbb{B}\left(0, r_{0}\right)$. Moreover, there exists a constant $M>0$ such that

$$
\left\|\theta_{\mu, \eta}^{*}\right\|_{\mathcal{L}\left(\mathfrak{F}^{s}(U, E)\right)} \leqslant M, \quad(\mu, \eta) \in \mathbb{B}\left(0, r_{0}\right) .
$$

(c) $\left[(\mu, \eta) \mapsto \theta_{\mu, \eta}^{*} u\right] \in C^{k}\left(\mathbb{B}\left(0, r_{0}\right), \mathfrak{F}^{s}(U, E)\right)$.

Proof. (a) The statement is immediate from (3.3) and [26, Proposition 2.2]. 
(b) We infer from (a) that

$$
\theta_{\mu, \eta}^{*} \circ \theta_{*}^{\mu, \eta}=\theta_{*}^{\mu, \eta} \circ \theta_{\mu, \eta}^{*}=\operatorname{id}_{\mathfrak{F}^{s}(U, E)} .
$$

[26, Proposition 2.4] implies that for $r$ small enough

$$
\theta_{\mu}^{*}, \bar{\theta}_{\eta}^{*} \in \mathcal{L} \operatorname{is}\left(\mathfrak{F}^{s}(U, E)\right), \quad(\mu, \eta) \in \mathbb{B}\left(0, r_{0}\right) .
$$

Moreover, there exists a constant $M>0$ such that

$$
\left\|\theta_{\mu}^{*}\right\|_{\mathcal{L}\left(\mathfrak{F}^{s}(U, E)\right)}+\left\|\bar{\theta}_{\eta}^{*}\right\|_{\mathcal{L}\left(\mathfrak{F}^{s}(U, E)\right)} \leqslant M, \quad(\mu, \eta) \in \mathbb{B}\left(0, r_{0}\right) .
$$

The assertion now follows from (3.3).

(c) It is a simple matter to see that the assertion holds for the transformation $\theta_{\mu}^{*}$ and $\bar{\theta}_{\eta}^{*}$, respectively, namely that we have

$$
\left[\mu \mapsto \theta_{\mu}^{*} u\right] \in C^{k}\left(\mathbb{B}\left(0, r_{0}\right), \mathfrak{F}^{s}(U, E)\right), \quad\left[\eta \mapsto \bar{\theta}_{\eta}^{*} u\right] \in C^{k}\left(\mathbb{B}\left(0, r_{0}\right), \mathfrak{F}^{s}(U, E)\right)
$$

with $\partial_{\mu}^{\alpha}\left[\theta_{\mu}^{*} u\right]=\chi_{m}^{|\alpha|} \varpi^{|\alpha|}\left[\theta_{\mu}^{*} \partial_{x}^{\alpha} u\right]$, and $\partial_{\eta}^{\beta}\left[\bar{\theta}_{\eta}^{*} u\right]=\chi_{m}^{\beta} \chi^{\beta}\left[\bar{\theta}_{\eta}^{*} \partial_{y}^{\beta} u\right]$. Then we can obtain from (3.3), (3.4), the point-wise multiplier theorem in [75, Section 3.3.2], and induction that the map

$$
(\mu, \eta) \mapsto \partial_{(\mu, \eta)}^{(\alpha, \beta)}\left[\theta_{\mu, \eta}^{*} u\right]=\chi_{m}^{|\alpha|+\beta} \chi^{\beta} \varpi^{|\alpha|}\left[\theta_{\mu, \eta}^{*} \partial_{(x, y)}^{(\alpha, \beta)} u\right], \quad|\alpha|+\beta \leqslant k,
$$

is separately $C^{k-|\alpha|-\beta}$-continuous into the space $\mathfrak{F}^{s}(U, E)$. So the case $k \in \mathbb{N}_{0} \cup\{\infty\}$ follows immediately from a well-known fact in multi-variable calculus. When $k=\omega$, the proof is basically the same as that of [26, Proposition 3.2].

Let

$$
Q:=\sum_{|\alpha| \leqslant l} a_{\alpha} \partial^{\alpha},
$$

be an $l$-th order linear differential operator on $U$ with coefficients $a_{\alpha}: U \rightarrow \mathcal{L}(E)$, where $\alpha \in$ $\mathbb{N}_{0}^{m+1}$. We set $\tilde{Q}_{\mu, \eta}:=\theta_{\mu, \eta}^{*} \propto \theta_{*}^{\mu, \eta}$.

THEOREM 3.2 Suppose that $a_{\alpha} \in C^{n+k}(O, \mathcal{L}(E)) \cap B U C^{n}(U, \mathcal{L}(E))$. Then

$$
\left[(\mu, \eta) \mapsto \tilde{\mathbb{Q}}_{\mu, \eta}\right] \in C^{k}\left(\mathbb{B}\left(0, r_{0}\right), \mathcal{L}\left(\mathfrak{F}^{s+l}(U, E), \mathfrak{F}^{s}(U, E)\right)\right),
$$

where $\mathfrak{F} \in\left\{b c, B U C, W_{p}, H_{p}\right\}$, and $s \in[0, n]$.

Proof. By modifying the proof of [26, Proposition 4.1] in an obvious way, one may verify the case of constant coefficients. Now the assertion is a direct consequence of point-wise multiplication results, and Proposition 3.1(c).

\subsection{Parameter-dependent diffeomorphisms on $\Omega$ and $\Omega_{i}$}

By means of $\theta_{\mu, \eta}$, we are now in a position to introduce a family of localized translations on $\Omega$ and $\Omega_{i}$, respectively, by

$$
\Theta_{\mu, \eta}(z)= \begin{cases}\psi_{\mathfrak{e}, \kappa_{c}} \circ \theta_{\mu, \eta} \circ \varphi_{\mathfrak{e}, \kappa_{c}}(z), & z \in \mathrm{O}_{\mathfrak{e}, \kappa_{c}}, \\ z, & z \in \Omega \backslash \mathrm{O}_{\mathfrak{e}, \kappa_{c}} .\end{cases}
$$


Thanks to Proposition 3.1(a), $\Theta_{\mu, \eta} \in \operatorname{Diff}^{\infty}(\Omega) \cap \operatorname{Diff}^{\infty}\left(\Omega_{i}\right)$ is evident.

There is a universal extension operator $\mathscr{E}_{i} \in \mathcal{L}\left(\mathfrak{F}^{s}\left(\Omega_{i}, E\right), \mathfrak{F}^{s}(\Omega, E)\right)$. The restriction operator from $\mathfrak{F}^{s}(\Omega, E)$ to $\mathfrak{F}^{s}\left(\Omega_{i}, E\right)$ is denoted by $\mathscr{R}_{i}$. Then $\mathscr{R}_{i}$ is a retraction from $\mathfrak{F}^{s}(\Omega, E)$ onto $\mathfrak{F}^{s}\left(\Omega_{i}, E\right)$ with $\mathscr{E}_{i}$ as a coretraction. When $u \in \mathfrak{F}^{s}(\Omega \backslash \Sigma, E)$, it is understood that $\mathscr{E}_{i} u:=\mathscr{E}_{i}\left(\left.u\right|_{\Omega_{i}}\right)$.

For $u \in E^{\Omega}$, its pull-back and push-forward by $\Theta_{\mu, \eta}$ can be explicitly expressed as

$$
\Theta_{\mu, \eta}^{*} u=\varphi_{\mathfrak{e}, \kappa_{c}}^{*} \theta_{\mu, \eta}^{*} \psi_{\mathfrak{e}, \kappa_{c}}^{*}\left(\zeta_{\mathfrak{e}} u\right)+\left(1_{\Omega}-\zeta_{\mathfrak{e}}\right) u, \quad \Theta_{*}^{\mu, \eta} u=\varphi_{\mathfrak{e}, \kappa_{c}}^{*} \theta_{*}^{\mu, \eta} \psi_{\mathfrak{e}, \kappa_{c}}^{*}\left(\zeta_{\mathfrak{e}} u\right)+\left(1_{\Omega}-\varsigma_{\mathfrak{e}}\right) u .
$$

Analogously, given $u \in E^{\Omega_{i}}$ of $u$, we have

$$
\Theta_{\mu, \eta}^{*} u=\mathscr{R}_{i} \circ \Theta_{\mu, \eta}^{*} \circ \mathscr{E}_{i} u, \quad \Theta_{*}^{\mu, \eta} u=\mathscr{R}_{i} \circ \Theta_{*}^{\mu, \eta} \circ \mathscr{E}_{i} u
$$

The construction of $\Theta_{\mu, \eta}$ implies that $\Theta_{\mu, \eta}^{*}\left(E^{\Omega_{i}}\right) \subset E^{\Omega_{i}}$, and likewise for $\Theta_{*}^{\mu, \eta}$. Suppose that $\mathfrak{F} \in\left\{b c, B C, W_{p}, H_{p}\right\}$. We define

$$
\mathfrak{F}_{\mathrm{cp}}^{s, \Omega}:=\left\{u \in \mathfrak{F}^{s}(\Omega, E): \operatorname{supp}(u) \subset \psi_{\mathfrak{e}, \kappa_{c}}\left(\overline{\mathbb{B}}_{5 \varepsilon_{0}, 17 a / 18}\right)\right\},
$$

and

$$
\mathfrak{F}_{\mathrm{cp}}^{s}:=\left\{u \in \mathfrak{F}^{s}\left(\mathbb{R}^{m+1}, E\right): \operatorname{supp}(u) \subset \overline{\mathbb{B}}_{5 \varepsilon_{0}, 17 a / 18}\right\} .
$$

The analogue of [73, Lemma 3.1] is at our disposal, i.e., it holds that

LEMMA $3.3 \varphi_{\mathfrak{e}, \kappa_{c}}^{*} \in \mathcal{L} \operatorname{is}\left(\mathfrak{F}_{\mathrm{cp}}^{s}, \mathfrak{F}_{\mathrm{cp}}^{s, \Omega}\right)$ with $\left[\varphi_{\mathfrak{e}, \kappa_{c}}^{*}\right]^{-1}=\psi_{\mathfrak{e}, \kappa_{c}}^{*}$.

Proof. Since [73, (3.2)] still holds with $\nabla$ and $g$ denoting the usual gradient and Euclidean metric in $\mathbb{R}^{m+1}$, respectively, the proof is essentially the same as that of [73, Lemma 3.1].

By means of Lemma 3.3, with only minor changes to the proofs in [73, Section 3], one can show that $\Theta_{\mu, \eta}$ inherits all the properties of its counterpart therein.

Proposition 3.4 Suppose that $\mathfrak{F} \in\left\{b c, B C, W_{p}, H_{p}\right\}$. Then

$$
\Theta_{\mu, \eta}^{*} \in \operatorname{Lis}\left(\mathfrak{F}^{s}(\Omega, E)\right) \cap \operatorname{Lis}\left(\mathfrak{F}^{s}\left(\Omega_{i}, E\right)\right), \quad\left[\Theta_{\mu, \eta}^{*}\right]^{-1}=\Theta_{*}^{\mu, \eta}, \quad(\mu, \eta) \in \mathbb{B}\left(0, r_{0}\right) .
$$

Moreover, there exists a constant $M>0$ such that

$$
\left\|\Theta_{\mu, \eta}^{*}\right\|_{\mathcal{L}\left(\mathfrak{F}^{s}(\Omega, E)\right)}+\left\|\Theta_{\mu, \eta}^{*}\right\|_{\mathcal{L}\left(\mathfrak{F}^{s}\left(\Omega_{i}, E\right)\right)} \leqslant M, \quad(\mu, \eta) \in \mathbb{B}\left(0, r_{0}\right) .
$$

Proof. The proof is almost the same as that of [73, Proposition 3.3]. For the reader's convenience, we will present a proof based on Lemma 3.3 for the case of $\Omega_{i}$, and the other case follows in a similar manner. The identity

$$
\Theta_{\mu, \eta}^{*} \circ \Theta_{*}^{\mu, \eta}=\Theta_{*}^{\mu, \eta} \circ \Theta_{\mu, \eta}^{*}=\operatorname{id}_{\mathfrak{F}^{s}(\Omega, E)} \cap \operatorname{id}_{\mathfrak{F}^{s}\left(\Omega_{i}, E\right)}
$$

is obvious from the definitions of $\Theta_{\mu, \eta}^{*}$ and $\Theta_{*}^{\mu, \eta}$ and $\theta_{\mu, \eta}$. In the formula

$$
\Theta_{\mu, \eta}^{*} u=\varphi_{\mathfrak{e}, \kappa_{c}}^{*} \theta_{\mu, \eta}^{*} \psi_{\mathfrak{e}, \kappa_{c}}^{*}\left(\varsigma_{\mathfrak{e}} u\right)+\left(1_{\Omega}-\varsigma_{\mathfrak{e}}\right) u
$$

it can easily verified that the multiplication operators $\zeta_{\mathfrak{e}}$ and $\left(1_{\Omega}-\zeta_{\mathfrak{e}}\right)$ are uniformly bounded, i.e.,

$$
\left\|\zeta_{\mathfrak{e}}\right\|_{\mathcal{L}\left(\mathfrak{F}^{s}(\Omega, E)\right)}+\left\|\left(1_{\Omega}-\zeta_{\mathfrak{e}}\right)\right\|_{\mathcal{L}\left(\mathfrak{F}^{s}(\Omega, E)\right)} \leqslant C_{1}
$$


for some constant $C_{1}$. Lemma 3.3 implies that for some constant $C_{2}$

$$
\left\|\varphi_{\mathfrak{e}, \kappa_{c}}^{*}\right\|_{\mathcal{L}\left(\mathfrak{F}_{\mathrm{cp}}^{s}, \mathfrak{\mho}_{\mathrm{cp}}^{s, \Omega}\right)}+\left\|\psi_{\mathfrak{e}, \kappa_{c}}^{*}\right\|_{\mathcal{L}\left(\mathfrak{F}_{\mathrm{cp}}^{s, \Omega}, \mathfrak{F}_{\mathrm{cp}}^{s}\right)} \leqslant C_{2}
$$

It follows from Proposition 3.1(b) that there is a uniform bound $C_{3}$ such that

$$
\left\|\theta_{\mu, \eta}^{*}\right\|_{\mathcal{L}\left(\mathfrak{F}_{\mathrm{cp}}^{s}\right)} \leqslant C_{3} .
$$

The statement then is a direct consequence of the open mapping theorem and (3.5).

\subsection{Time-dependence}

Let $J=[0, T], T>0$. Assume that $I \subset \stackrel{\circ}{J}$ is an open interval and $t_{c} \in I$ is a fixed point. Choose $\varepsilon_{0}$ so small that $\mathbb{B}\left(t_{c}, 3 \varepsilon_{0}\right) \subset \subset I$. Pick an auxiliary function

$$
\xi \in \Phi\left(\mathbb{B}\left(t_{c}, 2 \varepsilon_{0}\right),[0,1]\right) \quad \text { with }\left.\quad \xi\right|_{\mathbb{B}\left(t_{c}, \varepsilon_{0}\right)} \equiv 1 .
$$

The localized temporal translation is defined by

$$
\varrho_{\lambda}(t):=t+\xi(t) \lambda, \quad t \in J \text { and } \lambda \in \mathbb{R} .
$$

For $v: J \times \mathrm{Q}_{a} \rightarrow E$, the parameter-dependent diffeomorphism can be expressed as

$$
\tilde{v}_{\lambda, \mu, \eta}(t, \cdot):=\theta_{\lambda, \mu, \eta}^{*} v(t, \cdot)=\tilde{T}_{\mu, \eta}(t) \varrho_{\lambda}^{*} v(t, \cdot), \quad(\lambda, \mu, \eta) \in \mathbb{B}\left(0, r_{0}\right),
$$

where $\tilde{T}_{\mu, \eta}(t):=\theta_{\xi(t) \mu, \xi(t) \eta}^{*}$ for $t \in J$.

Given $v: J \times Q^{m} \rightarrow E$, analogously, setting $\tilde{T}_{\mu}(t):=\theta_{\xi(t) \mu}^{*}$, we can define

$$
\tilde{v}_{\lambda, \mu}(t, \cdot):=\theta_{\lambda, \mu}^{*} v(t, \cdot)=\tilde{T}_{\mu}(t) \varrho_{\lambda}^{*} v(t, \cdot), \quad(\lambda, \mu) \in \mathbb{B}\left(0, r_{0}\right) .
$$

It is understood that $\theta_{\mu}$ in (3.7) is restricted on the hyperplane $y=0$, appearing as a special case of (3.1).

For any $u \in J \times \Omega \rightarrow E$, or $u \in J \times \Omega_{i} \rightarrow E$, we define

$$
u_{\lambda, \mu, \eta}(t, \cdot):=\Theta_{\lambda, \mu, \eta}^{*} u(t, \cdot):=T_{\mu, \eta}(t) \varrho_{\lambda}^{*} u(t, \cdot), \quad(\lambda, \mu, \eta) \in \mathbb{B}\left(0, r_{0}\right) .
$$

Here $T_{\mu, \eta}(t):=\Theta_{\xi(t) \mu, \xi(t) \eta}^{*}$ for $t \in J$. By Proposition 3.4, for every $t, T_{\mu, \eta}(t)$ is invertible.

Let $\Theta_{*}^{\lambda, \mu, \eta}:=\left[\Theta_{\lambda, \mu, \eta}^{*}\right]^{-1}$. Note that $u_{\lambda, \mu, \eta}(0, \cdot)=u(0, \cdot)$.

The following lemma strengthens [26, Lemma 5.1].

LEMma 3.5 Suppose that $[(\mu, \eta) \mapsto f(\mu, \eta)] \in C^{k+j}\left(\mathbb{B}\left(0, r_{0}\right), X\right)$ for $j=0,1$ and some Banach space $X$. Let $F(\mu, \eta)(t):=f(\xi(t)(\mu, \eta))$ with $t \in J$. Then

$$
[(\mu, \eta) \mapsto F(\mu, \eta)] \in C^{k}\left(\mathbb{B}\left(0, r_{0}\right), C^{j}(J, X)\right) .
$$

Proof. To economize notation, we let $v:=(\mu, \eta)$. The case $j=0$ is proved in [26, Lemma 5.1]. We will only treat the case $k=\omega$ and $j=1$. The remaining cases follow in a similar way. 
Given $v_{0} \in \mathbb{B}\left(0, r_{0}\right)$, for every $v \in \mathbb{B}\left(v_{0}, r\right)$ with $r$ small enough

$$
f(v)=\sum_{\beta} \frac{1}{\beta !} \partial^{\beta} f\left(v_{0}\right)\left(v-v_{0}\right)^{\beta}, \quad \beta \in \mathbb{N}_{0}^{m+1},
$$

and there exist constants $M, R$ such that

$$
\left\|\partial^{\beta} f(v)\right\| \leqslant M \frac{\beta !}{R^{|\beta|}}, \quad v \in \mathbb{B}\left(v_{0}, r\right), \quad \beta \in \mathbb{N}_{0}^{m+1} .
$$

The constants $r, M, R$ depend continuously on $v_{0}$. Thus we can find uniform constants $r, M, R$ for $\overline{\mathbb{B}}^{m+1}\left(0,\left|v_{0}\right|\right)$. For each $t \in J$ and $v \in \mathbb{B}\left(v_{0}, r\right)$, the following series

$$
\sum_{\beta} \frac{\xi^{|\beta|}(t)}{\beta !} \partial^{\beta} f\left(\xi(t) v_{0}\right)\left(v-v_{0}\right)^{\beta}
$$

converges in $X$ and represents $F(v)(t)$. The temporal derivatives can be computed as follows. For $\beta \neq 0$,

$$
\begin{aligned}
& \left\|\frac{d}{d t}\left[\xi^{|\beta|}(t) \partial^{\beta} f\left(\xi(t) v_{0}\right)\right]\right\|_{C(J, X)} \\
& \quad \leqslant\left\|\xi^{\prime}(t)|\beta|\left\{\xi^{|\beta|-1}(t) \partial^{\beta} f\left(\xi(t) v_{0}\right)+\xi^{|\beta|}(t) \sum_{j=1}^{m+1} \partial^{\beta+e_{j}} f\left(\xi(t) v_{0}\right)\left(v_{0}\right)_{j}\right\}\right\|_{C(J, X)} \\
& \quad \leqslant M\|\xi\|_{1, \infty}\left(|\beta| \frac{\beta !}{R^{|\beta|}}+r_{0} \sum_{j=1}^{m+1} \frac{\left(\beta+e_{j}\right) !}{R^{|\beta|+1}}\right) \leqslant M^{\prime} \frac{\beta !}{(R / C)^{|\beta|}}
\end{aligned}
$$

for some sufficiently large $C$. Now the assertion follows right away.

Suppose that $\mathrm{O}_{\mathfrak{e}} \subset \mathbb{R}^{m+1}$ is an open subset containing $\psi_{\mathfrak{e}, \kappa_{c}}\left(\overline{\mathbb{B}}_{3 \varepsilon_{0}, 7 a / 9}\right)$. Put $\mathrm{O}_{\mathfrak{e}_{i}}:=\mathrm{O}_{\mathfrak{e}} \cap \Omega_{i}$.

When $k \in \mathbb{N}_{0} \cup\{\infty\}$, we say $u \in C^{k}\left(\mathrm{O}_{\mathfrak{e}_{i}}, E\right) \cap \mathfrak{F}^{s}\left(\Omega_{i}, E\right)$ if $\mathscr{E}_{i} u \in C^{k}\left(\mathrm{O}_{\mathfrak{e}}, E\right) \cap \mathfrak{F}^{s}(\Omega, E)$. By convention, $u \in C^{\omega}\left(\mathrm{O}_{\mathfrak{e}_{i}}, E\right) \cap \mathfrak{F}^{s}\left(\Omega_{i}, E\right)$ means that $u$ has an analytic extension $u^{e} \in C^{\omega}\left(\mathrm{O}_{\mathfrak{e}}, E\right) \cap$ $\mathfrak{F}^{s}(\Omega, E)$.

Proposition 3.6 Suppose that $(S, V) \in\left\{\left(\mathrm{O}_{\mathfrak{e}}, \Omega\right),\left(\mathrm{O}_{\mathfrak{e}_{i}}, \Omega_{i}\right)\right\}$.

(a) Suppose that $u \in C^{n+k+j}(S, E) \cap \mathfrak{F}^{s}(V, E)$ for $j=0,1$, where either $s \in[0, n]$ if $\mathfrak{F} \in$ $\left\{b c, W_{p}, H_{p}\right\}$, or $s=n$ if $\mathfrak{F}=B C$. Here $k \in \mathbb{N}_{0} \cup\{\infty\}$ if $(S, V)=\left(\mathrm{O}_{\mathfrak{e}_{i}}, \Omega_{i}\right)$. Then we have

$$
\left[(\mu, \eta) \mapsto T_{\mu, \eta} u\right] \in C^{k}\left(\mathbb{B}\left(0, r_{0}\right), C^{j}\left(J ; \mathfrak{F}^{s}(V, E)\right)\right)
$$

(b) Suppose that $Q=\sum_{|\alpha| \leqslant l} a_{\alpha} \partial^{\alpha}$ with $a_{\alpha} \in C^{n+k+j}(S, \mathcal{L}(E)) \cap B C^{n}(V, \mathcal{L}(E))$ for $j=0,1$.

Then

$$
\left[(\mu, \eta) \mapsto T_{\mu, \eta} Q T_{\mu, \eta}^{-1}\right] \in C^{k}\left(\mathbb{B}\left(0, r_{0}\right), C^{j}\left(J ; \mathcal{L}\left(\mathfrak{F}^{s+l}(V, E), \mathfrak{F}^{s}(V, E)\right)\right)\right),
$$

for $s \in[0, n]$ if $\mathfrak{F} \in\left\{B C, W_{p}, H_{p}\right\}$, or $s \in[0, n)$ if $\mathfrak{F}=b c$. 
Proof. (a) Modifying the proof of [73, Theorem 3.4] as in that of Proposition 3.4, one can show by means of Proposition 3.1(c) that

$$
\left[(\mu, \eta) \mapsto \Theta_{\mu, \eta}^{*} u\right] \in C^{k}\left(\mathbb{B}\left(0, r_{0}\right), \mathfrak{F}^{s}(V, E)\right) .
$$

Set $X=\mathfrak{F}^{s}(V, E), f(\mu, \eta)=\Theta_{\mu, \eta}^{*} u$. Now (a) is a direct consequence of Lemma 3.5.

(b) When $(S, V)=\left(\mathrm{O}_{\mathfrak{c}_{i}}, \Omega_{i}\right)$, let $Q_{i}:=\sum_{|\alpha| \leqslant l}\left(a_{\alpha}^{e}\right) \partial^{\alpha}$ on $\Omega$. Then

$$
Q_{\mu, \eta}=\mathscr{R}_{i} \circ \Theta_{\mu, \eta}^{*} Q_{i} \Theta_{*}^{\mu, \eta} \circ \mathscr{E}_{i} .
$$

The atlas $\mathfrak{A}_{\mathfrak{e}}$ is real analytically compatible with the Euclidean structure. Similar to the proof of [73, Proposition 3.6], by Theorem 3.2, we can show that

$$
\left[(\mu, \eta) \mapsto Q_{\mu, \eta}:=\Theta_{\mu, \eta}^{*} Q \Theta_{*}^{\mu, \eta}\right] \in C^{k}\left(\mathbb{B}\left(0, r_{0}\right), \mathcal{L}\left(\mathfrak{F}^{s+l}(V, E), \mathfrak{F}^{s}(V, E)\right)\right) .
$$

Set $X=\mathcal{L}\left(\mathfrak{F}^{s+l}(V, E), \mathfrak{F}^{s}(V, E)\right)$, and $f(\mu, \eta)=Q_{\mu, \eta}$. The assertion follows by Lemma 3.5.

Remark 3.7. Given any Banach space $X$, suppose that $u \in C^{n+k}(I, X) \cap \mathfrak{F}^{s}(J, X)$, where either $s \in[0, n]$ if $\mathfrak{F} \in\left\{b c, W_{p}, H_{p}\right\}$, or $s=n$ if $\mathfrak{F}=B C$. Following the proofs in [26, Section 3], we can verify that

$$
\left[\lambda \mapsto \varrho_{\lambda}^{*} u\right] \in C^{k}\left(\mathbb{B}\left(0, r_{0}\right), \mathfrak{F}^{s}(J, X)\right) .
$$

The localized translation $\Theta_{\mu, \eta}$, being restricted to $\Sigma$, induces a family of diffeomorphism $\left\{\Theta_{\mu}^{*}: \mu \in \mathbb{B}\left(0, r_{0}\right)\right\}$ on function spaces over $\Sigma$, which was introduced in [73]. For the reader's convenience, we will briefly state some of its properties herein.

$$
\Theta_{\mu}(q)= \begin{cases}\psi_{\kappa_{c}}\left(\theta_{\mu}\left(\varphi_{\kappa_{c}}(q)\right)\right), & q \in \mathrm{O}_{\kappa_{c}}, \\ q, & q \in \Sigma \backslash \mathrm{O}_{\kappa_{c}} .\end{cases}
$$

It is evident that $\Theta_{\mu} \in \operatorname{Diff}^{\infty}(\Sigma)$ for $\mu \in \mathbb{B}\left(0, r_{0}\right)$ with sufficiently small $r_{0}$. Given $u \in E^{\Sigma}, \Theta_{\mu}^{*}$ and $\Theta_{*}^{\mu}$ can be explicitly expressed as

$$
\Theta_{\mu}^{*} u=\varphi_{\kappa_{c}}^{*} \theta_{\mu}^{*} \psi_{\kappa_{c}}^{*}(\varsigma u)+\left(1_{\Sigma}-\varsigma\right) u, \quad \Theta_{*}^{\mu} u=\varphi_{\kappa_{c}}^{*} \theta_{*}^{\mu} \psi_{\kappa_{c}}^{*}(\varsigma u)+\left(1_{\Sigma}-\varsigma\right) u,
$$

respectively. Given $u \in E^{J \times \Sigma}$, setting $T_{\mu}(t):=\Theta_{\xi(t) \mu}^{*}$, we define

$$
u_{\lambda, \mu}(t, \cdot):=\Theta_{\lambda, \mu}^{*} u(t, \cdot)=T_{\mu}(t) \varrho_{\lambda}^{*} u(t, \cdot), \quad(\lambda, \mu) \in \mathbb{B}\left(0, r_{0}\right) .
$$

Let $\Theta_{*}^{\lambda, \mu}:=\left[\Theta_{\lambda, \mu}^{*}\right]^{-1}$.

A linear operator $Q: C^{\infty}(\Sigma, E) \rightarrow E^{\Sigma}$ is called a linear differential operator of order $l$ on $\Sigma$ if in every local chart $\left(\mathrm{O}_{\kappa}, \varphi_{\kappa}\right)$ with $\kappa \in \mathfrak{K}$, there exists some linear differential operator defined on $\mathrm{Q}^{m}$

$$
\mathbb{Q}_{\kappa}(x, \partial):=\sum_{|\alpha| \leqslant l} a_{\alpha}^{\kappa}(x) \partial^{\alpha} \text {, with } a_{\alpha}^{\kappa} \in \mathcal{L}(E)^{\mathrm{Q}^{m}},
$$

called the local representation of $Q$ in $\left(\mathrm{O}_{\kappa}, \varphi_{\kappa}\right)$, such that for any $u \in C^{\infty}(\Sigma, E)$

$$
\psi_{\kappa}^{*}(Q u)=Q_{\kappa}\left(\psi_{\kappa}^{*} u\right), \quad \kappa \in \mathfrak{K} .
$$


Proposition 3.8 Let $\mathrm{O}:=\mathrm{O}_{\mathfrak{e}} \cap \Sigma$.

(a) Suppose that $u \in C^{n+k+j}(\Sigma, E) \cap \mathfrak{F}^{s}(\Sigma, E)$ for $j=0,1$, where either $s \in[0, n]$ if $\mathfrak{F} \in$ $\left\{b c, W_{p}, H_{p}\right\}$, or $s=n$ if $\mathfrak{F}=B C$. Then we have

$$
\left[\mu \mapsto T_{\mu} u\right] \in C^{k}\left(\mathbb{B}\left(0, r_{0}\right), C^{j}\left(J ; \mathfrak{F}^{s}(\Sigma, E)\right)\right) .
$$

(b) Suppose that $Q$ is a linear differential operator on $\Sigma$ of order $l$ satisfying that for all $|\alpha| \leqslant l$ and $\kappa \in \mathfrak{K}, a_{\alpha}^{\kappa} \in B C^{n}\left(\mathrm{Q}^{m}, \mathcal{L}(E)\right)$ and $a_{\alpha}^{\kappa_{c}} \in C^{n+k+j}\left(\varphi_{\kappa_{c}}(\mathrm{O}), \mathcal{L}(E)\right)$ for $j=0,1$. Then for $s \in[0, n]$ if $\mathfrak{F}=B C$, or $s \in[0, n)$ if $\mathfrak{F}=b c$, or $s \in(-\infty, n]$ if $\mathfrak{F} \in\left\{W_{p}, H_{p}\right\}$

$$
\left[\mu \mapsto T_{\mu} Q T_{\mu}^{-1}\right] \in C^{k}\left(\mathbb{B}\left(0, r_{0}\right), C^{j}\left(J ; \mathcal{L}\left(\mathfrak{F}^{s+l}(\Sigma, E), \mathfrak{F}^{s}(\Sigma, E)\right)\right)\right) .
$$

Proof. The proofs for (a) and the case $s \geqslant 0$ in (b) are given in [73, Section 3]. We will only treat the case $k=\omega$ when $s<0$, the other cases follow similarly. Firstly, we show that for any $s \in(-\infty, 0)$ and $\mathfrak{F}_{p} \in\left\{W_{p}, H_{p}\right\}$

$$
\left[\mu \mapsto \theta_{\mu}^{*} \partial_{j} \theta_{*}^{\mu}\right] \in C^{\omega}\left(\mathbb{B}\left(0, r_{0}\right), \mathcal{L}\left(\mathfrak{F}_{p}^{s+1}\left(\mathbb{R}^{m}, E\right), \mathfrak{F}_{p}^{s}\left(\mathbb{R}^{m}, E\right)\right)\right) .
$$

(i) $s \leqslant-1$. On account that with $p^{\prime}$ denoting the Hölder duality of $p$,

$$
\partial_{j} \in \mathcal{L}\left(\mathfrak{F}_{p}^{s+1}\left(\mathbb{R}^{m}, E\right), \mathfrak{F}_{p}^{s}\left(\mathbb{R}^{m}, E\right)\right), \quad \text { and } \mathfrak{F}_{p}^{s}\left(\mathbb{R}^{m}, E\right)=\left(\mathfrak{F}_{p^{\prime}}^{-s}\left(\mathbb{R}^{m}, E\right)\right)^{\prime},
$$

for every $u \in \mathfrak{F}_{p}^{s+1}\left(\mathbb{R}^{m}, E\right)$ and $v \in \mathfrak{F}_{p^{\prime}}^{-s}\left(\mathbb{R}^{m}, E\right)$, we have

$$
\begin{aligned}
& \left\langle\theta_{\mu}^{*} \partial_{j} \theta_{*}^{\mu} u, v\right\rangle:=-\left\langle u, \theta_{\mu}^{*} \partial_{j}\left[\theta_{*}^{\mu} v\left|\operatorname{det}\left(D\left(\theta_{\mu}\right)^{-1}\right)\right|\right]\left|\operatorname{det}\left(D \theta_{\mu}\right)\right|\right\rangle \\
& \quad=-\left\langle u, \theta_{\mu}^{*} \partial_{j} \theta_{*}^{\mu} v \theta_{\mu}^{*}\left(\operatorname{det}\left(D\left(\theta_{\mu}\right)^{-1}\right)\right) \operatorname{det}\left(D \theta_{\mu}\right)\right\rangle-\left\langle u, v \theta_{\mu}^{*} \partial_{j}\left[\operatorname{det}\left(D\left(\theta_{\mu}\right)^{-1}\right)\right] \operatorname{det}\left(D \theta_{\mu}\right)\right\rangle .
\end{aligned}
$$

Here $\langle\cdot, \cdot\rangle$ denotes the duality pairing from $\mathfrak{F}_{p}^{s}\left(\mathbb{R}^{m}, E\right) \times \mathfrak{F}_{p^{\prime}}^{-s}\left(\mathbb{R}^{m}, E\right)$ to $\mathbb{R}$. By [26, Proposition 4.1], we have

$$
\left[\mu \mapsto \theta_{\mu}^{*} \partial_{j} \theta_{*}^{\mu}\right] \in C^{\omega}\left(\mathbb{B}\left(0, r_{0}\right), \mathcal{L}\left(\mathfrak{F}_{p^{\prime}}^{-s}\left(\mathbb{R}^{m}, E\right), \mathfrak{F}_{p^{\prime}}^{-s-1}\left(\mathbb{R}^{m}, E\right)\right)\right) .
$$

It is a simple matter to check that $\theta_{\mu}^{*}\left(\operatorname{det}\left(D\left(\theta_{\mu}\right)^{-1}\right)\right) \operatorname{det}\left(D \theta_{\mu}\right)=1$. One may compute

$$
\begin{aligned}
-\theta_{\mu}^{*} \partial_{j}[\operatorname{det} & \left.\left(D\left(\theta_{\mu}\right)^{-1}\right)\right] \operatorname{det}\left(D \theta_{\mu}\right) \\
= & -\theta_{\mu}^{*}\left[\partial_{j}\left[\operatorname{det}\left(D\left(\theta_{\mu}\right)^{-1}\right)\right] \theta_{*}^{\mu} \operatorname{det}\left(D \theta_{\mu}\right)\right]=\theta_{\mu}^{*} \partial_{j} \theta_{*}^{\mu} \operatorname{det}\left(D \theta_{\mu}\right) \theta_{\mu}^{*} \operatorname{det}\left(D\left(\theta_{\mu}\right)^{-1}\right) .
\end{aligned}
$$

We immediately have for all $k \in \mathbb{N}_{0}$ that

$$
\left[\mu \mapsto \operatorname{det}\left(D \theta_{\mu}\right)\right] \in C^{\omega}\left(\mathbb{B}\left(0, r_{0}\right), B C^{k+1}\left(\mathbb{R}^{m}\right)\right) .
$$

It follows again from [26, Proposition 4.1] that

$$
\left[\mu \mapsto \theta_{\mu}^{*} \partial_{j} \theta_{*}^{\mu} \operatorname{det}\left(D \theta_{\mu}\right)\right] \in C^{\omega}\left(\mathbb{B}\left(0, r_{0}\right), B C^{k}\left(\mathbb{R}^{m}\right)\right) .
$$

On the other hand, we obtain

$$
\left[\mu \mapsto \theta_{\mu}^{*} \operatorname{det}\left(D\left(\theta_{\mu}\right)^{-1}\right)\right]=1 / \operatorname{det}\left(D \theta_{\mu}\right) \in C^{\omega}\left(\mathbb{B}\left(0, r_{0}\right), B C^{k}\left(\mathbb{R}^{m}\right)\right) .
$$


Therefore, for every $u \in \mathfrak{F}_{p}^{s+1}\left(\mathbb{R}^{m}, E\right)$ and $v \in \mathfrak{F}_{p^{\prime}}^{-s}\left(\mathbb{R}^{m}, E\right)$,

$$
\left[\mu \mapsto\left\langle\theta_{\mu}^{*} \partial_{j} \theta_{*}^{\mu} u, v\right\rangle\right] \in C^{\omega}\left(\mathbb{B}\left(0, r_{0}\right)\right) .
$$

Now [13, Proposition 1] implies that

$$
\left[\mu \mapsto \theta_{\mu}^{*} \partial_{j} \theta_{*}^{\mu}\right] \in C^{\omega}\left(\mathbb{B}\left(0, r_{0}\right), \mathcal{L}\left(\mathfrak{F}_{p}^{s+1}\left(\mathbb{R}^{m}, E\right), \mathfrak{F}_{p}^{s}\left(\mathbb{R}^{m}, E\right)\right)\right) .
$$

(ii) $s \in(-1,0)$. [26, Proposition 4.1] and (i) show that

$$
\begin{aligned}
{\left[\mu \mapsto \theta_{\mu}^{*} \partial_{j} \theta_{*}^{\mu}\right] \in C^{\omega}\left(\mathbb{B}\left(0, r_{0}\right), \mathcal{L}\left(\mathfrak{F}_{p}^{s+2}\left(\mathbb{R}^{m}, E\right), \mathfrak{F}_{p}^{s+1}\left(\mathbb{R}^{m}, E\right)\right)\right) } \\
\cap C^{\omega}\left(\mathbb{B}\left(0, r_{0}\right), \mathcal{L}\left(\mathfrak{F}_{p}^{s}\left(\mathbb{R}^{m}, E\right), \mathfrak{F}_{p}^{s-1}\left(\mathbb{R}^{m}, E\right)\right)\right) .
\end{aligned}
$$

Thus for any $\mu_{0} \in \mathbb{B}\left(0, r_{0}\right)$, there exists some constants $r_{i}, M_{i}$ and $R_{i}$ with $i=1,2$ such that for all $\mu \in \mathbb{B}^{m}\left(\mu_{0}, r_{i}\right)$

$$
\left\|\frac{\partial^{\alpha}}{\partial \mu^{\alpha}}\left[\theta_{\mu}^{*} \partial_{j} \theta_{*}^{\mu}\right]\right\|_{X_{i}} \leqslant M_{i} \frac{\alpha !}{R_{i}^{|\alpha|}}, \quad \alpha \in \mathbb{N}_{0}^{m}
$$

Here $X_{1}:=\mathcal{L}\left(\mathfrak{F}_{p}^{s+2}, \mathfrak{F}_{p}^{s+1}\right)$ and $X_{2}:=\mathcal{L}\left(\mathfrak{F}_{p}^{s}, \mathfrak{F}_{p}^{s-1}\right)$. It follows from interpolation theory that $[\mu \mapsto$ $\left.\theta_{\mu}^{*} \partial_{j} \theta_{*}^{\mu}\right] \in C^{\infty}\left(\mathbb{B}\left(0, r_{0}\right), \mathcal{L}\left(\mathfrak{F}_{p}^{s+1}\left(\mathbb{R}^{m}, E\right), \mathfrak{F}_{p}^{s}\left(\mathbb{R}^{m}, E\right)\right)\right)$. Indeed, we have for $h=1,2, \cdots, m$ and any $\mu \in \mathbb{B}\left(0, r_{0}\right)$

$$
\left\|\frac{\theta_{\mu+t e_{h}}^{*} \partial_{j} \theta_{*}^{\mu+t e_{h}}-\theta_{\mu}^{*} \partial_{j} \theta_{*}^{\mu}}{t}-\frac{\partial}{\partial \mu_{h}} \theta_{\mu}^{*} \partial_{j} \theta_{*}^{\mu}\right\|_{X_{i}} \rightarrow 0, \quad t \rightarrow 0, \quad i=1,2 .
$$

By interpolation theory, the above limits converge when $X_{i}$ is replaced by $X:=$ $\mathcal{L}\left(\mathfrak{F}_{p}^{s+1}\left(\mathbb{R}^{m}, E\right), \mathfrak{F}_{p}^{s}\left(\mathbb{R}^{m}, E\right)\right)$. Continuity, or even $C^{\infty}$-smoothness, of $\theta_{\mu}^{*} \partial_{j} \theta_{*}^{\mu}$ in $X$ can be verified analogously.

A similar Cauchy estimate to (3.9) holds for $X$ by interpolation theory, i.e., for any $\mu_{0} \in$ $\mathbb{B}\left(0, r_{0}\right)$, there exists some constants $r=\min r_{i}, M=\max M_{i}$ and $R=\min R_{i}$ such that for all $\mu \in \mathbb{B}^{m}\left(\mu_{0}, r\right)$

$$
\left\|\frac{\partial^{\alpha}}{\partial \mu^{\alpha}}\left[\theta_{\mu}^{*} \partial_{j} \theta_{*}^{\mu}\right]\right\|_{\mathcal{L}\left(\mathfrak{F}_{p}^{s+1}, \mathfrak{F}_{p}^{s}\right)} \leqslant M \frac{\alpha !}{R^{|\alpha|}}, \quad \alpha \in \mathbb{N}_{0}^{m} .
$$

It is well-known that this estimate implies that

$$
\left[\mu \mapsto \theta_{\mu}^{*} \partial_{j} \theta_{*}^{\mu}\right] \in C^{\omega}\left(\mathbb{B}\left(0, r_{0}\right), \mathcal{L}\left(\mathfrak{F}_{p}^{s+1}\left(\mathbb{R}^{m}, E\right), \mathfrak{F}_{p}^{s}\left(\mathbb{R}^{m}, E\right)\right)\right) .
$$

(iii) For $s \in(-\infty, n]$, in view of the proofs for [26, Proposition 4.1, Theorem 4.2], we thus infer that for any linear differential operator $\tilde{\mathscr{Q}}=\sum_{|\alpha| \leqslant l} a_{\alpha} \partial^{\alpha}$, if $a_{\alpha} \in B C^{n}\left(\mathbb{R}^{m}, \mathcal{L}(E)\right) \cap$ $C^{n+k+j}(O, \mathcal{L}(E))$ for $O:=\varphi_{\kappa_{c}}(\mathrm{O})$, then

$$
\left[\mu \mapsto \theta_{\mu}^{*} \tilde{\mathbb{a}} \theta_{*}^{\mu}\right] \in C^{\omega}\left(\mathbb{B}\left(0, r_{0}\right), \mathcal{L}\left(\mathfrak{F}_{p}^{s+l}\left(\mathbb{R}^{m}, E\right), \mathfrak{F}_{p}^{s}\left(\mathbb{R}^{m}, E\right)\right)\right) .
$$


A similar proof to (i) shows that [73, Lemma 3.1] still holds for Sobolev-Slobodeckii and Bessel potential spaces of negative order. Indeed, let

$$
\mathfrak{F}_{\mathrm{cp}}^{s, \Sigma}:=\left\{u \in \mathfrak{F}^{s}(\Sigma, E): \operatorname{supp}(u) \subset \psi_{\kappa_{c}}\left(\overline{\mathbb{B}}\left(x_{c}, 5 \varepsilon_{0}\right)\right)\right\}
$$

and

$$
\mathfrak{F}_{\mathrm{cp}}^{s, \mathbb{R}^{m}}:=\left\{u \in \mathfrak{F}^{s}\left(\mathbb{R}^{m}, E\right): \operatorname{supp}(u) \subset \overline{\mathbb{B}}\left(x_{c}, 5 \varepsilon_{0}\right)\right\} .
$$

Pick $\overline{\mathbb{B}}\left(x_{c}, 5 \varepsilon_{0}\right) \subset \stackrel{\circ}{U} \subset \subset \mathrm{Q}^{m}$ with $U$ closed, and $\tau \in \Phi(\stackrel{\circ}{U},[0,1])$ with $\left.\tau\right|_{\overline{\mathbb{B}}\left(x_{c}, 5 \varepsilon_{0}\right)} \equiv 1$. For $r>0$, given any $u \in W_{p \text {, cp }}^{r}$ and $v \in W_{p^{\prime}}^{-r}\left(\mathbb{R}^{m}, E\right)$, we have

$$
\begin{aligned}
\left|\left\langle u, \varphi_{\kappa_{c}}^{*} v\right\rangle_{\Sigma}\right| & =\left|\left\langle\psi_{\kappa_{c}}^{*} u, \tau v \sqrt{|\operatorname{det} G|}\right\rangle\right| \\
& \leqslant M\left\|\psi_{\kappa_{c}}^{*} u\right\|_{W_{p}^{r}\left(\mathbb{R}^{m}\right)}\|v\|_{W_{p^{\prime}}^{-r}\left(\mathbb{R}^{m}\right)} \leqslant M\|u\|_{W_{p}^{r}(\Sigma)}\|v\|_{W_{p^{\prime}}^{-r}\left(\mathbb{R}^{m}\right)} .
\end{aligned}
$$

Here $G$ is the local matrix expression of the metric $g$, and $\langle\cdot, \cdot\rangle_{\Sigma}$ denotes the duality pairing from $\mathfrak{F}_{p}^{r}(\Sigma, E) \times \mathfrak{F}_{p^{\prime}}^{-r}(\Sigma, E)$ to $\mathbb{R}$. The ultimate line follows from the point-wise multiplier theorem, see [5, Section 9], and a similar assertion to [73, Lemma 3.1]. Thus the constant $M$ is independent of $u$ and $v$. It implies that for $r \in \mathbb{R}$

$$
\varphi_{\kappa_{c}}^{*} \in \operatorname{Lis}\left(\mathfrak{F}_{\mathrm{cp}}^{r, \mathbb{R}^{m}}, \mathfrak{F}_{\mathrm{cp}}^{r, \Sigma}\right), \quad \text { with } \quad\left[\varphi_{\kappa_{c}}^{*}\right]^{-1}=\psi_{\kappa_{c}}^{*} .
$$

Modifying the proof [73, Proposition 3.6] in an obvious way and applying Lemma 3.5, we have proved the statement of (b).

Recall that $\partial_{v}=\partial_{v_{\Sigma}}$.

PRoposition $3.9 T_{\mu} \partial_{\nu} T_{\mu, \eta}^{-1}=\partial_{\nu}$ on $H_{p}^{1}\left(\Omega_{i}\right)$.

Proof. It suffices to show that $\Theta_{\mu}^{*} \partial_{\nu} \Theta_{*}^{\mu, \eta}=\partial_{\nu}$. On account of

$$
\psi_{\kappa}^{*}\left(\partial_{\nu} u\right)=\psi_{\kappa}^{*}\left(v_{\Sigma} \cdot \nabla u\right)=\partial_{m+1} \psi_{\kappa}^{*} \mathscr{E}_{i} u
$$

for any $u \in H_{p}^{1}\left(\Omega_{i}\right)$, one readily computes

$$
\begin{aligned}
& \Theta_{\mu}^{*} \partial_{\nu} \Theta_{*}^{\mu, \eta} u=\Theta_{\mu}^{*} \partial_{\nu} \varphi_{\mathfrak{e}, \kappa_{c}}^{*} \theta_{*}^{\mu} \psi_{\mathfrak{e}, \kappa_{c}}^{*} \zeta_{\mathfrak{e}} \mathscr{E}_{i} u+\Theta_{\mu}^{*} \partial_{\nu}\left(1-\varsigma_{\mathfrak{e}}\right) u \\
& =\varphi_{\kappa_{c}}^{*} \theta_{\mu}^{*} \psi_{\kappa_{c}}^{*} \varsigma \partial_{\nu} \varphi_{\mathfrak{e}, \kappa_{c}}^{*} \theta_{*}^{\mu} \psi_{\mathfrak{e}, \kappa_{c}}^{*} \varsigma_{\mathfrak{e}} \mathscr{E}_{i} u+(1-\varsigma) \partial_{\nu} \varphi_{\mathfrak{e}, \kappa_{c}}^{*} \theta_{*}^{\mu} \psi_{\mathfrak{e}, \kappa_{c}}^{*} \varsigma_{\mathfrak{e}} \mathscr{E}_{i} u+\partial_{\nu}\left(1-\varsigma_{\mathfrak{e}}\right) u \\
& =\varphi_{\kappa_{c}}^{*} \theta_{\mu}^{*} \psi_{\kappa_{c}}^{*} \varsigma \varphi_{\kappa_{c}}^{*} \partial_{m+1} \theta_{*}^{\mu} \psi_{\mathfrak{e}, \kappa_{c}}^{*} \varsigma_{\mathfrak{e}} \mathscr{E}_{i} u \\
& +(1-\varsigma) \varphi_{\kappa_{c}}^{*} \partial_{m+1} \theta_{*}^{\mu} \psi_{\mathfrak{e}, \kappa_{c}}^{*} \varsigma_{\mathfrak{e}} \mathscr{E}_{i} u+\partial_{v}\left(1-\varsigma_{\mathfrak{e}}\right) u \\
& =\varphi_{\kappa_{c}}^{*} \theta_{\mu}^{*} \psi_{\kappa_{c}}^{*} \varsigma \varphi_{\kappa_{c}}^{*} \theta_{*}^{\mu} \partial_{m+1} \psi_{\mathfrak{e}, \kappa_{c}}^{*} \varsigma_{\mathfrak{e}} \mathscr{E}_{i} u+\varphi_{\kappa_{c}}^{*} \theta_{\mu}^{*} \psi_{\kappa_{c}}^{*}(1-\varsigma) \varphi_{\kappa_{c}}^{*} \theta_{*}^{\mu} \partial_{m+1} \psi_{\mathfrak{e}, \kappa_{c}}^{*} \varsigma_{\mathfrak{e}} \mathscr{E}_{i} u \\
& +\partial_{v}\left(1-\varsigma_{\mathfrak{e}}\right) u \\
& =\varphi_{\kappa_{c}}^{*} \partial_{m+1} \psi_{\mathfrak{e}, \kappa_{c}}^{*} \zeta_{\mathfrak{e}} \mathscr{E}_{i} u+\partial_{\nu}\left(1-\varsigma_{\mathfrak{e}}\right) u=\partial_{\nu} u \text {. }
\end{aligned}
$$

In the above, we have used the fact that $\Theta_{\mu}^{*} \partial_{\nu}\left(1-\varsigma_{\mathfrak{e}}\right) u=\left(1-\varsigma_{\mathfrak{e}}\right) u$.

Recall definitions (3.6) and (3.7). The following proposition shows that the space $\mathbb{E}(J)$ is invariant under the transformation $\Theta_{\lambda, \mu, \eta}^{*}$ and $\Theta_{\lambda, \mu}^{*}$, respectively. 
Proposition $3.10\left[(\vartheta, h) \mapsto\left(\Theta_{\lambda, \mu, \eta}^{*} \vartheta, \Theta_{\lambda, \mu}^{*} h\right)\right] \in \operatorname{Sis}(\mathbb{E}(J)) \cap \operatorname{Lis}\left({ }_{0} \mathbb{E}(J)\right)$ with $(\lambda, \mu, \eta) \in$ $\mathbb{B}\left(0, r_{0}\right)$. Moreover, there exist some $B_{\lambda, \mu, \eta}, B_{\lambda, \mu}$ satisfying

$$
\left\{\begin{array}{l}
{\left[(\lambda, \mu, \eta) \mapsto B_{\lambda, \mu, \eta}\right] \in C^{\omega}\left(\mathbb{B}\left(0, r_{0}\right), \mathcal{L}\left(\mathbb{E}_{1}(J), \mathbb{F}_{1}(J)\right)\right),} \\
{\left[(\lambda, \mu) \mapsto B_{\lambda, \mu}\right] \in C^{\omega}\left(\mathbb{B}\left(0, r_{0}\right), \mathcal{L}\left(\mathbb{E}_{2}(J), \mathbb{F}_{2}(J)\right)\right),}
\end{array}\right.
$$

such that

$$
\left\{\begin{array}{l}
\partial_{t}\left[\vartheta_{\lambda, \mu, \eta}\right]=\left(1+\xi^{\prime} \lambda\right) \Theta_{\lambda, \mu, \eta}^{*} \partial_{t} \vartheta+B_{\lambda, \mu, \eta}\left(\vartheta_{\lambda, \mu, \eta}\right) \\
\partial_{t}\left[h_{\lambda, \mu}\right]=\left(1+\xi^{\prime} \lambda\right) \Theta_{\lambda, \mu}^{*} \partial_{t} h+B_{\lambda, \mu}\left(h_{\lambda, \mu}\right) .
\end{array}\right.
$$

In particular, $B_{\lambda, 0,0}=0$ and $B_{\lambda, 0}=0$.

Proof. (i) Following the proof of [26, Proposition 2.4] and interpolation theory, we can show that for any Banach space $X, \varrho_{\lambda}^{*} \in \mathcal{L i s}(\mathfrak{F}(I, X))$ and $\left[\varrho_{\lambda}^{*}\right]^{-1}=\varrho_{*}^{\lambda}$ with $\mathfrak{F} \in\left\{B C^{s}, W_{p}^{s}\right\}$. In particular, there exists $M>0$ such that $\left\|\varrho_{\lambda}^{*}\right\|_{\mathcal{L}(\mathfrak{F}(I, X))} \leqslant M$ for $\lambda \in \mathbb{B}$. A similar estimate as in [26, Lemma 8.3] by using the intrinsic norms of Besov spaces reveals that

$$
\left[(u, \rho) \mapsto\left(\tilde{u}_{\lambda, \mu, \eta}, \tilde{\rho}_{\lambda, \mu}\right)\right] \in \operatorname{Lis}\left(\mathbb{E}_{1}\left(J, \mathbb{R}^{m+1} ; E\right) \times \mathbb{E}_{2}\left(J, \mathbb{R}^{m} ; E\right)\right) .
$$

(ii) Observe that

$$
\left(\Theta_{\lambda, \mu, \eta}^{*} \vartheta, \Theta_{\lambda, \mu}^{*} h\right)(0)=(\vartheta, h)(0), \quad \partial_{t}\left(\Theta_{\lambda, \mu, \eta}^{*} \vartheta, \Theta_{\lambda, \mu}^{*} h\right)(0)=\partial_{t}(\vartheta, h)(0),
$$

whenever the corresponding derivative exists. For any $z \in \Omega \backslash O_{\mathfrak{e}, \kappa_{c}}, T_{\mu, \eta} \vartheta(z)=\vartheta(z)$, we conclude that $\partial_{\nu_{\Omega}} T_{\mu, \eta} \vartheta=0$. For every $t \in J, \vartheta(t) \in B C(\Omega)$. It follows from Proposition 3.4 that $T_{\mu, \eta}(t) \vartheta(t) \in B C(\Omega)$, which implies that $\llbracket T_{\mu, \eta}(t) \vartheta(t) \rrbracket=0$. Moreover, for $(\mu, \eta) \in \mathbb{B}\left(0, r_{0}\right)$

$$
\begin{aligned}
\left\|T_{\mu, \eta, \eta} \vartheta\right\|_{\mathbb{E}_{1}(J)} & =\sum_{i}\left\|\mathscr{R}_{i} \varphi_{\mathfrak{e}, \kappa_{c}}^{*} \tilde{T}_{\mu, \eta} \psi_{\mathfrak{e}, \kappa_{c}}^{*}\left(\zeta_{\mathfrak{e}} \mathscr{E}_{i} \vartheta\right)+\left(1-\zeta_{\mathfrak{e}}\right) \vartheta\right\|_{\mathbb{E}_{1}(J)} \\
& \leqslant M \sum_{i}\left[\left\|\tilde{T}_{\mu, \eta} \psi_{\mathfrak{e}, \kappa_{c}}^{*}\left(\zeta_{\mathfrak{e}} \mathscr{E}_{i} \vartheta\right)\right\|_{\mathbb{E}_{1}\left(J, \mathbb{R}^{m+1} ; E\right)}+\left\|\left(1-\zeta_{\mathfrak{e}}\right) \mathscr{E}_{i} \vartheta\right\|_{\mathbb{E}_{1}(J)}\right] \leqslant M\|\vartheta\|_{\mathbb{E}_{1}(J)}
\end{aligned}
$$

The last line follows from (i), Lemma 3.3 and point-wise multiplier theorem, see [75, Section 3.3.2]. In virtue of [73, Lemma 3.1], one can show similarly that

$$
\left[h \mapsto T_{\mu} h\right] \in \mathcal{L}\left(\mathbb{E}_{2}(J)\right), \quad \mu \in \mathbb{B}\left(0, r_{0}\right) .
$$

Then it is a direct consequence of the open mapping theorem that

$$
\left[(\vartheta, h) \mapsto\left(\Theta_{\lambda, \mu, \eta}^{*} \vartheta, \Theta_{\lambda, \mu}^{*} h\right)\right] \in \operatorname{Lis}(\mathbb{E}(J)) .
$$

By (3.10), the statement $\left[(\vartheta, h) \mapsto\left(T_{\mu, \eta} \vartheta, T_{\mu} h\right)\right] \in \mathcal{L} \operatorname{is}\left({ }_{0} \mathbb{E}(J)\right)$ is immediate. 
(iii) The temporal derivative of $\vartheta_{\lambda, \mu, \eta}$ can be computed as follows.

$$
\begin{aligned}
\partial_{t}\left[\vartheta_{\lambda, \mu, \eta}\right]= & \left(1-\varsigma_{\mathfrak{e}}\right) \partial_{t}\left[\varrho_{\lambda}^{*} \vartheta\right]+\sum_{i} \mathscr{R}_{i} \varphi_{\mathfrak{e}, \kappa_{c}}^{*} \partial_{t}\left[\tilde{T}_{\mu, \eta} \psi_{\mathfrak{e}, \kappa_{c}}^{*}\left(\varsigma_{\mathfrak{e}} \varrho_{\lambda}^{*} \mathscr{E}_{i} \vartheta\right)\right] \\
= & \left(1-\varsigma_{\mathfrak{e}}\right)\left(1+\xi^{\prime} \lambda\right) \varrho_{\lambda}^{*} \partial_{t} \vartheta+\sum_{i}\left\{\mathscr{R}_{i} \varphi_{\mathfrak{e}, \kappa_{c}}^{*} \tilde{T}_{\mu, \eta} \psi_{\mathfrak{e}, \kappa_{c}}^{*}\left(\varsigma_{\mathfrak{e}} \partial_{t}\left[\varrho_{\lambda}^{*} \mathscr{E}_{i} \vartheta\right]\right)\right. \\
& +\mathscr{R}_{i} \sum_{j=1}^{m} \varphi_{\mathfrak{e}, \kappa_{c}}^{*}\left(\xi^{\prime} \chi_{m} \varpi \mu_{j} \tilde{T}_{\mu, \eta} \partial_{j}\left[\psi_{\mathfrak{e}, \kappa_{c}}^{*} \varsigma_{\mathfrak{e}} \varrho_{\lambda}^{*} \mathscr{E}_{i} \vartheta\right]\right) \\
& \left.+\mathscr{R}_{i} \varphi_{\mathfrak{e}, \kappa_{c}}^{*}\left(\xi^{\prime} \chi_{m} \chi \eta \tilde{T}_{\mu, \eta} \partial_{m+1}\left[\psi_{\mathfrak{e}, \kappa_{c}}^{*} \zeta_{\mathfrak{e}} \varrho_{\lambda}^{*} \mathscr{E}_{i} \vartheta\right]\right)\right\} \\
= & \left(1+\xi^{\prime} \lambda\right) \Theta_{\lambda, \mu, \eta}^{*} \partial_{t} \vartheta+B_{\lambda, \mu, \eta}\left(\vartheta_{\lambda, \mu, \eta}\right) .
\end{aligned}
$$

The last line follows from the definition of $\Theta_{\lambda, \mu, \eta}^{*}$ and the equality

$$
\begin{aligned}
\mathscr{R}_{i} \varphi_{\mathfrak{e}, \kappa_{c}}^{*} \tilde{T}_{\mu, \eta} \psi_{\mathfrak{e}, \kappa_{c}}^{*}\left(\varsigma_{\mathfrak{e}} \partial_{t}\left[\varrho_{\lambda}^{*} \mathscr{E}_{i} \vartheta\right]\right)+\left(1-\varsigma_{\mathfrak{e}}\right)\left(1+\xi^{\prime} \lambda\right) \varrho_{\lambda}^{*} \partial_{t} \vartheta \\
=\mathscr{R}_{i} \varphi_{\mathfrak{e}, \kappa_{c}}^{*} \tilde{T}_{\mu, \eta} \psi_{\mathfrak{e}, \kappa_{c}}^{*}\left[\varsigma_{\mathfrak{e}}\left(1+\xi^{\prime} \lambda\right) \varrho_{\lambda}^{*} \partial_{t} \mathscr{E}_{i} \vartheta\right]+\left(1-\varsigma_{\mathfrak{e}}\right)\left(1+\xi^{\prime} \lambda\right) \varrho_{\lambda}^{*} \partial_{t} \vartheta \\
=\left(1+\xi^{\prime} \lambda\right) \Theta_{\lambda, \mu, \eta}^{*} \partial_{t} \vartheta .
\end{aligned}
$$

Given $u \in H_{p}^{1}(\Omega \backslash \Sigma)$, the term $B_{\lambda, \mu, \eta}$ can be explicitly written as

$$
\begin{aligned}
B_{\lambda, \mu, \eta}(u)=\sum_{i}\left\{\sum_{j=1}^{m} \mathscr{R}_{i} \varphi_{\mathfrak{e}, \kappa_{c}}^{*}\left(\xi^{\prime} \chi_{m} \varpi \mu_{j} \tilde{T}_{\mu, \eta} \partial_{j}\left[\psi_{\mathfrak{e}, \kappa_{c}}^{*} \varsigma_{\mathfrak{e}} T_{\mu, \eta}^{-1} \mathscr{E}_{i} u\right]\right)\right\} \\
\quad+\mathscr{R}_{i} \varphi_{\mathfrak{e}, \kappa_{c}}^{*}\left(\xi^{\prime} \chi_{m} \chi \eta \tilde{T}_{\mu, \eta} \partial_{m+1}\left[\psi_{\mathfrak{e}, \kappa_{c}}^{*} \varsigma_{\mathfrak{e}} T_{\mu, \eta}^{-1} \mathscr{E}_{i} u\right]\right) \\
=\sum_{i}\left\{\sum_{j=1}^{m} \mathscr{R}_{i} \xi^{\prime} \varphi_{\mathfrak{e}, \kappa_{c}}^{*} \chi_{m} \varphi_{\mathfrak{e}, \kappa_{c}}^{*} \varpi \mu_{j} T_{\mu, \eta} Q^{j} T_{\mu, \eta, \mathscr{E}_{i}}^{-1} u\right. \\
\left.+\mathscr{R}_{i} \xi^{\prime} \varphi_{\mathfrak{e}, \kappa_{c}}^{*} \chi_{m} \varphi_{\mathfrak{e}, \kappa_{c}}^{*} \chi \eta T_{\mu, \eta} Q^{m+1} T_{\mu, \eta}^{-1} \mathscr{E}_{i} u\right\} .
\end{aligned}
$$

Here $Q^{j}$ with $j=1, \cdots, m+1$ are first order linear differential operators defined on $\Omega \backslash \Sigma$ compactly supported in $\mathrm{T}_{a, \kappa_{c}}$ satisfying $\psi_{\mathfrak{e}, \kappa_{c}}^{*} Q^{j} u=\varsigma_{\mathfrak{e}} \partial_{j} \psi_{\mathfrak{e}, \kappa_{c}}^{*} u$ for all $u \in H_{p}^{1}(\Omega \backslash \Sigma)$. By means of Proposition 3.6(b) and the real analytic compatibility of the atlas $\mathfrak{A}_{\mathfrak{e}}$ with the Euclidean structure, it is not hard to check that

$$
\left[(\lambda, \mu, \eta) \mapsto B_{\lambda, \mu, \eta}\right] \in C^{\omega}\left(\mathbb{B}\left(0, r_{0}\right), C^{1}\left(J ; \mathcal{L}\left(W_{p}^{s+1}(\Omega \backslash \Sigma), W_{p}^{s}(\Omega \backslash \Sigma)\right)\right)\right) .
$$

In [73, Proposition 3.10], it is shown that

$$
\partial_{t}\left[h_{\lambda, \mu}\right]=\left(1+\xi^{\prime} \lambda\right) \Theta_{\lambda, \mu}^{*} \partial_{t} h+B_{\lambda, \mu}\left(h_{\lambda, \mu}\right)
$$

with $B_{\lambda, 0}=0$ and

$$
\left[(\lambda, \mu) \mapsto B_{\lambda, \mu}\right] \in C^{\omega}\left(\mathbb{B}\left(0, r_{0}\right), C^{1}\left(J ; \mathcal{L}\left(W_{p}^{s+1}(\Sigma), W_{p}^{s}(\Sigma)\right)\right)\right) .
$$

This completes the proof. 
With minor modification of the above proof, we immediately have the following similar result. Proposition 3.11 For $(\lambda, \mu, \eta) \in \mathbb{B}\left(0, r_{0}\right)$ with sufficiently small $r_{0}>0$,

$$
\left[(f, g, q) \mapsto\left(f_{\lambda, \mu, \eta}, g_{\lambda, \mu}, q_{\lambda, \mu}\right)\right] \in \operatorname{Lis}\left(\prod_{i=1}^{3} \mathbb{F}_{i}(J), \prod_{i=1}^{3} \mathbb{F}_{i}(J)\right),
$$

and

$$
\left[(f, g, q) \mapsto\left(f_{\lambda, \mu, \eta}, g_{\lambda, \mu}, q_{\lambda, \mu}\right)\right] \in \operatorname{Lis}\left(\prod_{i=1}^{3}{ }_{0} \mathbb{F}_{i}(J), \prod_{i=1}^{3}{ }_{0} \mathbb{F}_{i}(J)\right) .
$$

The main theorem of this section is

THEOREM 3.12 Suppose that

$$
(\vartheta, h) \in B C(J \times \Omega, E) \times B C(J \times \Sigma, E) .
$$

Then

$$
(\vartheta, h) \in C^{k}\left(\stackrel{\circ}{J} \times \mathrm{T}_{a / 3} \backslash \Sigma, E\right) \times C^{k}(\stackrel{\circ}{J} \times \Sigma, E)
$$

iff for any $\left(t_{c}, z_{c}\right) \in \stackrel{\circ}{J} \times \mathrm{T}_{a / 3} \backslash \Sigma$, there exists some $r_{0}=r_{0}\left(t_{c}, z_{c}\right)>0$ and a corresponding family of parameter-dependent diffeomorphisms $\left\{\left(\Theta_{\lambda, \mu, \eta}^{*}, \Theta_{\lambda, \mu}^{*}\right):(\lambda, \mu, \eta) \in \mathbb{B}\left(0, r_{0}\right)\right\}$ such that

$$
\left[(\lambda, \mu, \eta) \mapsto\left(\vartheta_{\lambda, \mu, \eta}, h_{\lambda, \mu}\right)\right] \in C^{k}\left(\mathbb{B}\left(0, r_{0}\right), B C(J \times \Omega, E) \times B C(J \times \Sigma, E)\right) .
$$

Proof. The proof follows in a similar manner to that of [73, Theorem 3.5].

\section{Regularity of solutions to the Stefan Problem}

Throughout the rest of this paper, we assume that $\sigma>0$ and $d_{i}, \gamma \in C^{k+2}(0, \infty), \psi_{i} \in$ $C^{k+3}(0, \infty)$ for $i=1,2$ with $k \in \mathbb{N} \cup\{\infty, \omega\}$ such that

$$
\kappa_{i}(u)=-u \psi_{i}^{\prime \prime}(u)>0, \quad d_{i}(u)>0, \quad u \in(0, \infty),
$$

and

$$
\gamma(u)>0, \quad u \in(0, \infty), \quad \text { or } \quad \gamma \equiv 0 .
$$

Let $J:=[0, T]$. We define

$$
\begin{aligned}
& \mathbb{G}_{2}(J):=H_{p}^{1}\left(J ; W_{p}^{-1 / p}(\Sigma)\right) \cap L_{p}\left(J ; W_{p}^{2-1 / p}(\Sigma)\right) \\
& \mathbb{G}_{3}(J):=H_{p}^{1}\left(J ; W_{p}^{-1-1 / p}(\Sigma)\right) \cap L_{p}\left(J ; W_{p}^{1-1 / p}(\Sigma)\right),
\end{aligned}
$$

and

$$
\begin{aligned}
& \mathbb{C}_{1}(J):=C(J \times \bar{\Omega}) \cap C\left(J ; C^{1}\left(\bar{\Omega}_{i}\right)\right) \\
& \mathbb{C}_{2}(J):=C\left(J ; C^{3}(\Sigma)\right) \cap C^{1}(J ; C(\Sigma)) .
\end{aligned}
$$

It is shown in $[65$, Section 3$]$ that

$$
\mathbb{E}_{1}(J) \hookrightarrow \mathbb{C}_{1}(J), \quad \mathbb{E}_{2}(J) \hookrightarrow \mathbb{C}_{2}(J) .
$$

Observe that the constants in these embeddings blow up as $T \rightarrow 0$, however, they are uniform in $T$ if one considers the space ${ }_{0} \mathbb{E}(J)$ ! 


\subsection{Linearization at a real analytic temperature and the initial interface}

Suppose that $\hat{z}_{0}=\left(\hat{\vartheta}_{0}, \hat{h}_{0}\right) \in \mathbb{F}_{4}$ satisfies $\hat{\vartheta}_{0}>0$. Given any $\varepsilon>0$, by the Weierstrass approximation theorem, we can find some

$$
\vartheta_{A} \in C^{\omega}(\bar{\Omega}), \quad\left\|\vartheta_{A}-\hat{\vartheta}_{0}\right\|_{B C(\Omega)} \leqslant \varepsilon
$$

For sufficiently small $\varepsilon, \vartheta_{A}>0$, and when $\gamma \equiv 0, l\left(\vartheta_{A}\right) \neq 0$. Let

$$
\begin{aligned}
& \kappa_{A}(x)=\kappa\left(\vartheta_{A}(x)\right), \quad d_{A}(x)=d\left(\vartheta_{A}(x)\right), \quad l_{A}(x)=l\left(\vartheta_{A}(x)\right), \quad \sigma_{0}=\frac{\sigma}{m}, \\
& l_{1}(t, \cdot)=\llbracket \psi^{\prime}\left(e^{\Delta_{\Sigma} t} \vartheta_{A}\right) \rrbracket, \quad \gamma_{1}(t, \cdot)=\gamma\left(e^{\Delta_{\Sigma} t} \vartheta_{A}\right) .
\end{aligned}
$$

For a function $\vartheta \in \mathbb{E}_{1}(J)$, we do not distinguish $\left.\vartheta\right|_{\Sigma}$ from $\vartheta$ if the choice is self-evident from the context. Here $\Delta_{\Sigma}$ denotes the Laplace-Beltrami operator on $\Sigma$. Similarly, we define

$$
\begin{aligned}
& \hat{\kappa}_{0}(x)=\kappa\left(\hat{\vartheta}_{0}(x)\right), \quad \hat{d}_{0}(x)=d\left(\hat{\vartheta}_{0}(x)\right), \quad \hat{l}_{0}(x)=l\left(\hat{\vartheta}_{0}(x)\right), \quad \hat{\gamma}_{0}=\gamma\left(\hat{\vartheta}_{0}\right), \\
& \hat{l}_{1}(t, \cdot)=\llbracket \psi^{\prime}\left(e^{\Delta_{\Sigma} t} \hat{\vartheta}_{0}\right) \rrbracket, \quad \hat{\gamma}_{1}(t, \cdot)=\gamma\left(e^{\Delta_{\Sigma} t} \hat{\vartheta}_{0}\right) .
\end{aligned}
$$

For $z=(\vartheta, h) \in \mathbb{E}(J)$, we define

$$
\begin{aligned}
& F(z)=\left(\kappa_{A}-\kappa(\vartheta)\right) \partial_{t} \vartheta+\left(d(\vartheta)-d_{A}\right) \Delta \vartheta-d(\vartheta) M_{2}(h): \nabla^{2} \vartheta \\
& +d^{\prime}(\vartheta)\left|\left(I-M_{1}(h)\right) \nabla \vartheta\right|^{2}-d(\vartheta)\left(M_{3}(h) \mid \nabla \vartheta\right)+\kappa(\vartheta) R(h) \vartheta, \\
& G(z)=-(\llbracket \psi(\vartheta) \rrbracket+\sigma \mathcal{H}(h))+l_{1} \vartheta+\sigma_{0} \Delta_{\Sigma} h+\left(\gamma(\vartheta) \beta(h)-\gamma_{1}\right) \partial_{t} h, \\
& Q(z)=\llbracket\left(d(\vartheta)-d_{A}\right) \partial_{\nu} \vartheta \rrbracket+\left(l_{A}-l(\vartheta)\right) \partial_{t} h-\left(\llbracket d(\vartheta) \nabla \vartheta \rrbracket \mid M_{4}(h) \nabla_{\Sigma} h\right) \\
& +\gamma(\vartheta) \beta(h)\left(\partial_{t} h\right)^{2} \text {. }
\end{aligned}
$$

Here we have

$$
\begin{aligned}
& M_{2}(h)=M_{1}(h)+M_{1}^{\top}(h)-M_{1}(h) M_{1}^{\top}(h), \\
& M_{3}(h)=\left(I-M_{1}(h)\right): \nabla M_{1}(h), \\
& M_{4}(h)=\left(I-M_{1}(h)\right)^{\top} M_{0}(h) .
\end{aligned}
$$

Employing the above notations and splitting into the principal linear part and a nonlinear part, we arrive at the following formulation of problem (2.1)

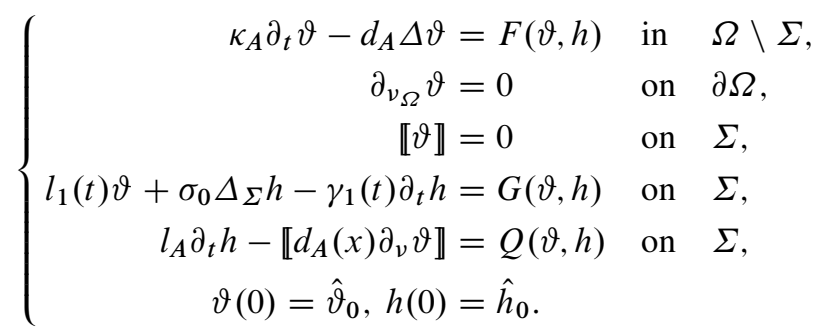

We assume that $\hat{z}_{0}$ further satisfies the compatibility conditions (ee), that is, when $\gamma \equiv 0$

$$
\partial_{\nu_{\Omega}} \hat{\vartheta}_{0}=0, \quad l_{1}(0) \hat{\vartheta}_{0}+\sigma_{0} \Delta_{\Sigma} \hat{h}_{0}=G\left(\hat{z}_{0}\right), \quad Q\left(\hat{z}_{0}\right)+\llbracket d_{A} \partial_{\nu} \hat{\vartheta}_{0} \rrbracket \in W_{p}^{2-6 / p}(\Sigma),
$$


and when $\gamma>0$

$$
\partial_{\nu_{\Omega}} \hat{\vartheta}_{0}=0, \quad l_{A} l_{1}(0) \hat{\vartheta}_{0}+l_{A} \sigma_{0} \Delta_{\Sigma} \hat{h}_{0}-\gamma_{1}(0) \llbracket d_{A} \partial_{v} \hat{\vartheta}_{0} \rrbracket=\gamma_{1}(0) Q\left(\hat{z}_{0}\right)+l_{A} G\left(\hat{z}_{0}\right) .
$$

In the definition of $\left(G\left(\hat{z}_{0}\right), Q\left(\hat{z}_{0}\right)\right)$, it is understood that $\partial_{t} h(0)$ is replaced by

$$
\partial_{t} h(0)= \begin{cases}\frac{1}{\hat{l}_{0}}\left(\llbracket \hat{d}_{0} \partial_{\nu} \hat{\vartheta}_{0} \rrbracket-\left(\llbracket \hat{d}_{0} \nabla \hat{\vartheta}_{0} \rrbracket \mid M_{4}\left(\hat{h}_{0}\right) \nabla_{\Sigma} \hat{h}_{0}\right)\right), & \gamma \equiv 0, \\ \frac{1}{\beta\left(\hat{h}_{0}\right) \hat{\gamma}_{0}}\left(\llbracket \psi\left(\hat{\vartheta}_{0}\right) \rrbracket+\sigma \mathcal{H}\left(\hat{h}_{0}\right)\right), & \gamma>0 .\end{cases}
$$

When $\gamma \equiv 0$, we also need to impose the well-posedness condition $l\left(\hat{\vartheta}_{0}\right) \neq 0$ on $\Gamma_{0}$.

\subsection{Regularity of a special solution}

In this section, we prove the analyticity of the solution to a linearized Stefan problem with initial data $\hat{z}_{0}$, that is, we consider regularity of solutions to the following equation.

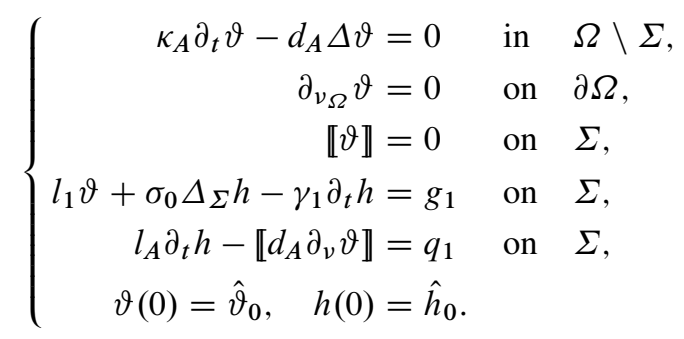

Here $g_{1}:=e^{\Delta_{\Sigma} t} G\left(\hat{z}_{0}\right)$ and $q_{1}:=e^{\Delta_{\Sigma} t} Q\left(\hat{z}_{0}\right)$. Recall definition (2.2). We set

$$
\begin{aligned}
\mathbb{F}(J)=\left\{\left(f, g, q,\left(\vartheta_{0}, h_{0}\right)\right)\right. & \in \mathbb{F}_{1}(J) \times \mathbb{F}_{2}(J) \times \mathbb{F}_{3}(J) \times \mathbb{F}_{4}: \\
& \left.\left.\left(f, g, q,\left(\vartheta_{0}, h_{0}\right)\right) \text { satisfies the linear compatibility conditions (LCe }\right)\right\},
\end{aligned}
$$

where $\left(f, g, q,\left(\vartheta_{0}, h_{0}\right)\right)$ is said to satisfy the linear compatibility conditions $(\mathcal{L C C})$ if

$$
\begin{array}{ll}
\partial_{\nu_{\Omega}} \vartheta_{0}=0, & l_{1}(0) \vartheta_{0}+\sigma_{0} \Delta_{\Sigma} h_{0}=g(0), \quad q(0)+\llbracket d_{A} \partial_{\nu} \vartheta_{0} \rrbracket \in W_{p}^{2-6 / p}(\Sigma), \quad \gamma \equiv 0, \\
\partial_{\nu_{\Omega}} \vartheta_{0}=0, & l_{A} l_{1}(0) \vartheta_{0}+l_{A} \sigma_{0} \Delta_{\Sigma} h_{0}-\gamma_{1}(0) \llbracket d_{A} \partial_{\nu} \vartheta_{0} \rrbracket=\gamma_{1}(0) q(0)+l_{A} g(0), \gamma>0 .
\end{array}
$$

We equipped $\mathbb{F}(J)$ with the following norm

$$
\begin{aligned}
& \left\|\left(f, g, q,\left(\vartheta_{0}, h_{0}\right)\right)\right\|_{\mathbb{F}(J)} \\
:= & \|f\|_{\mathbb{F}_{1}(J)}+\|g\|_{\mathbb{F}_{2}(J)}+\|q\|_{\mathbb{F}_{3}(J)}+\left\|\left(\vartheta_{0}, h_{0}\right)\right\|_{\mathbb{F}_{4}}+(1-\operatorname{sgn}(\gamma))\left\|q(0)+\llbracket d_{A} \partial_{\nu} \vartheta_{0} \rrbracket\right\|_{W_{p}^{2-6 / p}(\Sigma)} .
\end{aligned}
$$

(i) Regularity of $g_{1}$ and $q_{1}$.

Case 1: $\gamma \equiv 0$. It is not hard to check that

$$
l_{1}, \gamma_{1} \in \mathbb{F}_{2}(J), \quad \kappa_{A} \in W_{p}^{1}(\Omega \backslash \Sigma), \quad d_{A} \in W_{p}^{2-2 / p}(\Omega \backslash \Sigma), \quad l_{A} \in W_{p}^{2-3 / p}(\Sigma) .
$$


Indeed, the first term can be obtained as follows. $\left.\vartheta_{A}\right|_{\Sigma} \in W_{p}^{2-3 / p}(\Sigma)$ and the $L_{p}$-maximal regularity of $\Delta_{\Sigma}$ imply that

$$
e^{\Delta_{\Sigma} t} \vartheta_{A} \in H_{p}^{1}\left(J ; W_{p}^{-1 / p}(\Sigma)\right) \cap L_{p}\left(J ; W_{p}^{2-1 / p}(\Sigma)\right) .
$$

By [55, Proposition 3.2], we infer that $e^{\Delta_{\Sigma} t} \vartheta_{A} \in \mathbb{F}_{2}(J)$. Now the first term in (4.5) follows from the regularity of $l, \gamma$. Similar to (4.5), one checks that

$$
\hat{l}_{1}, \hat{\gamma}_{1} \in \mathbb{F}_{2}(J), \quad \hat{\kappa}_{0} \in W_{p}^{1}(\Omega \backslash \Sigma), \quad \hat{d}_{0} \in W_{p}^{2-2 / p}(\Omega \backslash \Sigma), \quad \hat{l}_{0} \in W_{p}^{2-3 / p}(\Sigma) .
$$

In [72], an analysis of the structure of the mean curvature operator $\mathcal{H}(h)$ and $\beta(h)$ is obtained, which shows that $\mathcal{H}(h)$ is a rational function in the height function $h$ and its spatial derivatives up to second order, while $\beta(h)$ is a rational function in $h$ and its first order spatial derivatives. On account of the embeddings $W_{p}^{3-3 / p}(\Sigma) \hookrightarrow C(\Sigma)$ and $W_{p}^{2-3 / p}(\Sigma) \hookrightarrow C(\Sigma)$, we conclude from [75, Theorem 2.8.3] and a similar argument to [74, Proposition 2.7] that $W_{p}^{3-3 / p}(\Sigma)$ and $W_{p}^{2-3 / p}(\Sigma)$ are multiplication algebras under point-wise multiplication. Since

$$
\left[x \mapsto x^{a}\right] \in C^{\omega}((0, \infty)), \quad a \in \mathbb{R},
$$

well-known results for substitution operators for Sobolev-Slobodeckii spaces imply that

$$
\beta\left(\hat{h}_{0}\right) \in W_{p}^{3-3 / p}(\Sigma), \quad \mathcal{H}\left(\hat{h}_{0}\right) \in W_{p}^{2-3 / p}(\Sigma) .
$$

(4.5)-(4.7) then imply

$$
G\left(\hat{z}_{0}\right) \in W_{p}^{2-3 / p}(\Sigma)
$$

and a similar argument yields

$$
Q\left(\hat{z}_{0}\right) \in W_{p}^{1-3 / p}(\Sigma) .
$$

Together with the $L_{p}$-maximal regularity of $\Delta_{\Sigma}$ and [55, Proposition 3.2], we conclude from (4.8) and (4.9) that

$$
g_{1} \in \mathbb{G}_{2}(J) \hookrightarrow \mathbb{F}_{2}(J), \quad q_{1} \in \mathbb{G}_{3}(J) \hookrightarrow \mathbb{F}_{3}(J) .
$$

Case 2: $\gamma>0$. Based on the discussion in (i), it suffices to show the regularity of $\partial_{t} h(0)$, which is defined in (4.3). As illustrated in (i), we have

$$
\beta\left(\hat{h}_{0}\right) \hat{\gamma}_{0},\left(\llbracket \psi\left(\hat{\vartheta}_{0}\right) \rrbracket+\sigma \mathcal{H}\left(\hat{h}_{0}\right)\right) \in W_{p}^{2-3 / p}(\Sigma) .
$$

It implies that $\partial_{t} h(0) \in W_{p}^{2-3 / p}(\Sigma)$. It yields the desired results, i.e.,

$$
g_{1} \in \mathbb{F}_{2}(J), \quad q_{1} \in \mathbb{F}_{3}(J) .
$$

In virtue of condition (Ce) and the definitions of $g_{1}$ and $q_{1}$, one checks that condition (LCe) is at our disposal. Therefore, all the compatibility conditions in [65, Theorems 3.3,3.5] are satisfied, and then there exists a unique solution $z^{*}=\left(\vartheta^{*}, h^{*}\right) \in \mathbb{E}(J)$ to the linear system (4.4).

Remark 4.1. The compatibility condition $\llbracket d\left(\theta_{0}\right) \partial_{\nu_{\Gamma_{0}}} \theta_{0} \rrbracket \in W_{p}^{2-6 / p}\left(\Gamma_{0}\right)$ in Theorem 1.1 implies that

$$
\llbracket \hat{d}_{0} \partial_{\nu} \hat{\vartheta}_{0} \rrbracket-\left(\llbracket \hat{d}_{0} \nabla \hat{\vartheta}_{0} \rrbracket \mid M_{4}\left(\hat{h}_{0}\right) \nabla_{\Sigma} \hat{h}_{0}\right) \in W_{p}^{2-6 / p}(\Sigma)
$$


which is equivalent to

$$
\left(\llbracket \hat{d}_{0} \nabla \hat{\vartheta}_{0} \rrbracket \mid v_{\Sigma}-M_{4}\left(\hat{h}_{0}\right) \nabla_{\Sigma} \hat{h}_{0}\right) \in W_{p}^{2-6 / p}(\Sigma) .
$$

From the above discussion, we infer that

$$
\llbracket \nabla \hat{\vartheta}_{0} \rrbracket \in W_{p}^{2-6 / p}\left(\Sigma, \mathbb{R}^{m+1}\right) .
$$

Now based on this observation and (4.6), we conclude that

$$
Q\left(\hat{z}_{0}\right)+\llbracket d_{A} \partial_{v} \hat{\vartheta}_{0} \rrbracket \in W_{p}^{2-6 / p}(\Sigma) .
$$

The other two conditions in conditions (ee), i.e., when $\gamma \equiv 0$

$$
\partial_{\nu_{\Omega}} \hat{\vartheta}_{0}=0, \quad l_{1}(0) \hat{\vartheta}_{0}+\sigma_{0} \Delta_{\Sigma} \hat{h}_{0}=G\left(\hat{z}_{0}\right)
$$

and when $\gamma>0$

$$
\partial_{\nu_{\Omega}} \hat{\vartheta}_{0}=0, \quad l_{A} l_{1}(0) \hat{\vartheta}_{0}+l_{A} \sigma_{0} \Delta_{\Sigma} \hat{h}_{0}-\gamma_{1}(0) \llbracket d_{A} \partial_{\nu} \hat{\vartheta}_{0} \rrbracket=\gamma_{1}(0) Q\left(\hat{z}_{0}\right)+l_{A} G\left(\hat{z}_{0}\right) .
$$

can be easily obtained from the remaining compatibility conditions in Theorems 1.1 and 1.2. Similarly, the compatibility conditions in Theorems 1.1 and 1.2 can also be concluded from conditions (ee). By [65, Theorems 3.1, 3.2], problem (4.2) with initial data $\hat{z}_{0}$ has a unique $L_{p}$-solution on some possibly small but nontrivial interval $J:=[0, T]$, which is denoted by $\hat{z}=(\hat{\vartheta}, \hat{h})$.

Next, we apply the parameter-dependent diffeomorphisms $\Theta_{\lambda, \mu, \eta}^{*}$ and $\Theta_{\lambda, \mu}^{*}$ to show the analyticity of the solution $z^{*}$. We will use the following useful fact that for any time-independent map $\eta$ acting on $\mathfrak{F}^{s}\left(\Omega_{i}, E\right)$

$$
\Theta_{\lambda, \mu, \eta}^{*} \eta=T_{\mu, \eta} \eta T_{\mu, \eta}^{-1} \Theta_{\lambda, \mu, \eta}^{*},
$$

and a similar result also holds for $\Theta_{\lambda, \mu}^{*}$. By Proposition 3.10, we have

$$
\begin{aligned}
\partial_{t}\left[\vartheta_{\lambda, \mu, \eta}^{*}\right] & =\left(1+\xi^{\prime} \lambda\right) \Theta_{\lambda, \mu, \eta}^{*} \partial_{t} \vartheta^{*}+B_{\lambda, \mu, \eta}\left(\vartheta_{\lambda, \mu, \eta}^{*}\right) \\
& =\left(1+\xi^{\prime} \lambda\right) \Theta_{\lambda, \mu, \eta}^{*}\left(d_{A} / \kappa_{A}\right) \Delta \vartheta^{*}+B_{\lambda, \mu, \eta}\left(\vartheta_{\lambda, \mu, \eta}^{*}\right) \\
& =\left(1+\xi^{\prime} \lambda\right)\left(d_{A, \lambda, \mu, \eta} / \kappa_{A, \lambda, \mu, \eta}\right) T_{\mu, \eta} \Delta T_{\mu, \eta}^{-1} \vartheta_{\lambda, \mu, \eta}^{*}+B_{\lambda, \mu, \eta}\left(\vartheta_{\lambda, \mu, \eta}^{*}\right),
\end{aligned}
$$

and either by Proposition 3.9

$$
\begin{aligned}
\partial_{t}\left[h_{\lambda, \mu}^{*}\right] & =\left(1+\xi^{\prime} \lambda\right) \Theta_{\lambda, \mu}^{*} \partial_{t} h^{*}+B_{\lambda, \mu}\left(h_{\lambda, \mu}^{*}\right) \\
& =\left(1+\xi^{\prime} \lambda\right) \Theta_{\lambda, \mu}^{*}\left[\left(q_{1}+\llbracket d_{A} \partial_{\nu} \vartheta^{*} \rrbracket\right) / l_{A}\right]+B_{\lambda, \mu}\left(h_{\lambda, \mu}^{*}\right) \\
& =\left(1+\xi^{\prime} \lambda\right)\left\{\left(q_{1, \lambda, \mu} / l_{A, \lambda, \mu}\right)+\llbracket\left(d_{A, \lambda, \mu} / l_{A, \lambda, \mu}\right) \partial_{\nu} \vartheta_{\lambda, \mu}^{*} \rrbracket\right\}+B_{\lambda, \mu}\left(h_{\lambda, \mu}^{*}\right),
\end{aligned}
$$

when $\gamma \equiv 0$, or when $\gamma>0$, we have

$$
\begin{aligned}
\partial_{t}\left[h_{\lambda, \mu}^{*}\right] & =\left(1+\xi^{\prime} \lambda\right) \Theta_{\lambda, \mu}^{*}\left[\left(l_{1} \vartheta^{*}+\sigma_{0} \Delta_{\Sigma} h^{*}-g_{1}\right) / \gamma_{1}\right]+B_{\lambda, \mu}\left(h_{\lambda, \mu}^{*}\right) \\
& =\left(1+\xi^{\prime} \lambda\right)\left(l_{1, \lambda, \mu} \vartheta_{\lambda, \mu}^{*}+\sigma_{0} T_{\mu} \Delta_{\Sigma} T_{\mu}^{-1} h_{\lambda, \mu}^{*}-g_{1, \lambda, \mu}\right) /\left(\gamma_{1, \lambda, \mu}\right)+B_{\lambda, \mu}\left(h_{\lambda, \mu}^{*}\right) .
\end{aligned}
$$


We define a map $\Phi: \mathbb{E}(J) \times \mathbb{B}\left(0, r_{0}\right) \rightarrow \mathbb{F}(J):((\vartheta, h),(\lambda, \mu, \eta)) \mapsto$

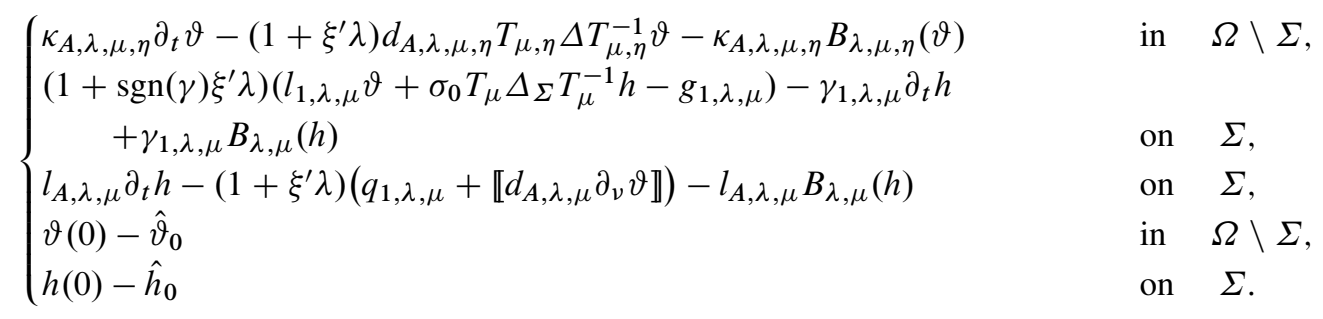

Setting $z_{\lambda, \mu, \eta}^{*}=\left(\vartheta_{\lambda, \mu, \eta}^{*}, h_{\lambda, \mu}^{*}\right)$, note that $\Phi\left(z_{\lambda, \mu, \eta}^{*},(\lambda, \mu, \eta)\right)=0$ for all $(\lambda, \mu, \eta) \in \mathbb{B}\left(0, r_{0}\right)$.

(ii) We need to show that $\Phi$ actually maps into $\mathbb{F}(J)$. For simplification, we set $\Phi(z,(\lambda, \mu, \eta))=$ $\left(f_{2}, g_{2}, q_{2},\left(\vartheta_{0}, h_{0}\right)\right)^{\top}$. It is obvious that based on trace theory of anisotropic Sobolev-Slobodeckii spaces, see [22, Section 2],

$$
\left(\vartheta_{0}, h_{0}\right) \in \mathbb{F}_{4} .
$$

By means of Propositions 3.6 and 3.10, we infer that $f_{2} \in \mathbb{F}_{1}(J)$. For the regularity of the next two terms, we split into two cases as before. When $\gamma \equiv 0$, observe that $\Delta_{\Sigma} \in \mathcal{L}\left(\mathbb{E}_{2}(J), \mathbb{F}_{2}(J)\right)$. Proposition 3.11, (4.5) and (4.10) yield

$$
\gamma_{1, \lambda, \mu}, l_{1, \lambda, \mu}, g_{1, \lambda, \mu} \in \mathbb{F}_{2}(J), \quad q_{1, \lambda, \mu} \in \mathbb{F}_{3}(J) .
$$

Taking into consideration that $\partial_{t} h \in \mathbb{F}_{3}(J)$ and the fact that $\mathbb{F}_{2}(J), \mathbb{F}_{3}(J)$ are multiplication algebras, we conclude from Propositions 3.8, 3.10 and (4.11) that

$$
g_{2} \in \mathbb{F}_{2}(J), \quad q_{2} \in \mathbb{F}_{3}(J) .
$$

When $\gamma>0$, since $\partial_{t} h \in \mathbb{F}_{2}(J)$, the desired regularity results clearly hold true. It remains to show condition (LCe).

Case 1: $\gamma \equiv 0$. It is immediate that $\partial_{\nu_{\Omega}} \vartheta_{0}=\partial_{\nu_{\Omega}}\left(\vartheta(0)-\hat{\vartheta}_{0}\right)=0$. One checks that

$$
\begin{aligned}
l_{1}(0) \vartheta_{0}+\sigma_{0} \Delta_{\Sigma} h_{0} & =l_{1}(0)\left(\vartheta(0)-\hat{\vartheta}_{0}\right)+\sigma_{0} \Delta_{\Sigma}\left(h(0)-\hat{h}_{0}\right) \\
& =l_{1, \lambda, \mu}(0) \vartheta(0)+\sigma T_{\mu}(0) \Delta_{\Sigma} T_{\mu}^{-1}(0) h(0)-g_{1, \lambda, \mu}(0)=g_{2}(0),
\end{aligned}
$$

by recalling $u_{\lambda, \mu}(0, \cdot)=u(0, \cdot)$ for any $u \in E^{\Sigma}$, and

$$
\begin{aligned}
q_{2}(0)+\llbracket d_{A, \lambda, \mu}(0) \partial_{\nu} \vartheta_{0} \rrbracket= & l_{A, \lambda, \mu}(0) \partial_{t} h(0)-q_{1, \lambda, \mu}(0)-\llbracket d_{A} \partial_{\nu} \vartheta(0) \rrbracket \\
& -l_{A, \lambda, \mu}(0) B_{\lambda, \mu}(h(0))+\llbracket d_{A} \partial_{\nu}\left(\vartheta(0)-\hat{\vartheta}_{0}\right) \rrbracket \\
= & l_{A} \partial_{t} h(0)-q_{1}(0)-l_{A} B_{\lambda, \mu}(h(0))-\llbracket d_{A} \partial_{\nu} \hat{\vartheta}_{0} \rrbracket .
\end{aligned}
$$

By the discussion in (ii), $q_{1}(0)+\llbracket d_{A} \partial_{\nu} \hat{\vartheta}_{0} \rrbracket \in W_{p}^{2-6 / p}(\Sigma)$. Since $W_{p}^{2-6 / p}(\Sigma)$ is a multiplication algebra, (3.12), (4.5) and [65, formula (3.1)] imply that

$$
q_{2}(0)+\llbracket d_{A, \lambda, \mu}(0) \partial_{\nu} \vartheta_{0} \rrbracket \in W_{p}^{2-6 / p}(\Sigma) .
$$


Case 2: $\gamma>0$.

$$
\begin{aligned}
l_{A} l_{1}(0) \vartheta_{0}+ & l_{A} \sigma_{0} \Delta_{\Sigma} h_{0}-\gamma_{1}(0) \llbracket d_{A} \partial_{\nu} \vartheta_{0} \rrbracket \\
= & l_{A, \lambda, \mu}(0) l_{1, \lambda, \mu}(0)\left(\vartheta(0)-\hat{\vartheta}_{0}\right)+l_{A, \lambda, \mu}(0) \sigma_{0} T_{\mu}(0) \Delta_{\Sigma} T_{\mu}^{-1}(0)\left(h(0)-\hat{h}_{0}\right) \\
\quad & \quad-\gamma_{1, \lambda, \mu}(0) \llbracket d_{A, \lambda, \mu} \partial_{\nu}\left(\vartheta(0)-\hat{\vartheta}_{0}\right) \rrbracket \\
= & l_{A} l_{1}(0) \vartheta(0)+l_{A} \sigma_{0} \Delta_{\Sigma} h(0)-\gamma_{1}(0) \llbracket d_{A} \partial_{\nu} \vartheta(0) \rrbracket-l_{A} g_{1}(0)-\gamma_{1}(0) q_{1}(0) \\
= & l_{A} g_{2}(0)+\gamma_{1}(0) q_{2}(0) .
\end{aligned}
$$

Therefore $\Phi(z,(\lambda, \mu, \eta)) \in \mathbb{F}(J)$.

(iii) Let $w=(u, \rho) \in \mathbb{E}(J)$. The Frèchet derivative of $\Phi$ with respect to $(\vartheta, h)$ at $\left(z^{*}, 0\right)$ is clearly given by

$$
D_{1} \Phi\left(z^{*}, 0\right) w=\left\{\begin{array}{lll}
\kappa_{A} \partial_{t} u-d_{A} \Delta u & \text { in } & \Omega \backslash \Sigma, \\
l_{1} u+\sigma_{0} \Delta_{\Sigma} \rho-\gamma_{1} \partial_{t} \rho & \text { on } & \Sigma, \\
l_{A} \partial_{t} \rho-\llbracket d_{A} \partial_{\nu} u \rrbracket & \text { on } & \Sigma, \\
u(0) & \text { in } & \Omega \backslash \Sigma, \\
\rho(0) & \text { on } & \Sigma .
\end{array}\right.
$$

It is obvious that

$$
D_{1} \Phi\left(z^{*}, 0\right) w \in \mathbb{F}_{1}(J) \times \mathbb{F}_{2}(J) \times \mathbb{F}_{3}(J) \times \mathbb{F}_{4} .
$$

Condition (LCe) can be verified as in (ii) by using [65, formula (3.1)] in the case $\gamma \equiv 0$. We can deduce from $[65$, Theorems $3.3,3.5]$ that

$$
D_{1} \Phi\left(z^{*}, 0\right) \in \operatorname{Lis}(\mathbb{E}(J), \mathbb{F}(J)) .
$$

(iv) Regularity of $\left[(\lambda, \mu) \mapsto\left(g_{1, \lambda, \mu}, q_{1, \lambda, \mu}\right)\right]$. We express $g_{1, \lambda, \mu}$ as

$$
\begin{aligned}
g_{1, \lambda, \mu} & =\Theta_{\lambda, \mu}^{*} e^{\Delta_{\Sigma} t} G\left(\hat{z}_{0}\right)=\Theta_{\lambda, \mu}^{*}\left(c+\Delta_{\Sigma}\right) e^{\Delta_{\Sigma} t}\left(c+\Delta_{\Sigma}\right)^{-1} G\left(\hat{z}_{0}\right) \\
& =T_{\mu}\left(c+\Delta_{\Sigma}\right) T_{\mu}^{-1} \Theta_{\lambda, \mu}^{*} e^{\Delta_{\Sigma} t}\left(c+\Delta_{\Sigma}\right)^{-1} G\left(\hat{z}_{0}\right) .
\end{aligned}
$$

For sufficiently large $c$, it is well-known that $\left(c+\Delta_{\Sigma}\right)$ is an isomorphism from $W_{p}^{s+2}(\Sigma)$ to $W_{p}^{s}(\Sigma)$ for any $s \in \mathbb{R}$. We consider the solution to

$$
\partial_{t} \rho+\Delta_{\Sigma} \rho=0, \quad \rho(0)=\left(c+\Delta_{\Sigma}\right)^{-1} G\left(\hat{z}_{0}\right) .
$$

$\hat{\rho}:=e^{\Delta_{\Sigma} t}\left(c+\Delta_{\Sigma}\right)^{-1} G\left(\hat{z}_{0}\right)$ is the unique solution to (4.12). Furthermore, Proposition 3.10 shows that $\hat{\rho}_{\lambda, \mu}$ satisfies

$$
\begin{aligned}
\partial_{t}\left[\hat{\rho}_{\lambda, \mu}\right] & =\left(1+\xi^{\prime} \lambda\right) \Theta_{\lambda, \mu}^{*} \partial_{t} \hat{\rho}+B_{\lambda, \mu}\left(\hat{\rho}_{\lambda, \mu}\right) \\
& =-\left(1+\xi^{\prime} \lambda\right) T_{\mu} \Delta_{\Sigma} T_{\mu}^{-1} \hat{\rho}_{\lambda, \mu}+B_{\lambda, \mu}\left(\hat{\rho}_{\lambda, \mu}\right) .
\end{aligned}
$$

We define the map $\Phi_{g}: \mathbb{X}_{2}(J) \times \mathbb{B}\left(0, r_{0}\right) \rightarrow L_{p}\left(J ; W_{p}^{2-1 / p}(\Sigma)\right) \times W_{p}^{4-3 / p}(\Sigma)$ by

$$
\Phi_{g}(\rho,(\lambda, \mu))=\left(\begin{array}{c}
\partial_{t} \rho+\left(1+\xi^{\prime} \lambda\right) T_{\mu} \Delta_{\Sigma} T_{\mu}^{-1} \rho-B_{\lambda, \mu}(\rho) \\
\rho(0)-\left(c+\Delta_{\Sigma}\right)^{-1} G\left(\hat{z}_{0}\right)
\end{array}\right) .
$$


Here $\mathbb{X}_{2}(J):=H_{p}^{1}\left(J ; W^{2-1 / p}(\Sigma)\right) \cap L_{p}\left(J ; W^{4-1 / p}(\Sigma)\right)$. Note that $\Phi_{g}\left(\hat{\rho}_{\lambda, \mu},(\lambda, \mu)\right)=(0,0)^{\top}$. By the $L_{p}$-maximal regularity of $\Delta_{\Sigma}$, we immediately have

$$
D_{1} \Phi_{g}(\hat{\rho}, 0) \in \mathcal{L} \operatorname{is}\left(\mathbb{X}_{2}(J), L_{p}\left(J ; W_{p}^{2-1 / p}(\Sigma)\right) \times W_{p}^{4-3 / p}(\Sigma)\right)
$$

We define a bilinear and continuous map

$$
T: C\left(J ; \mathcal{L}\left(W_{p}^{4-1 / p}(\Sigma), W_{p}^{3-1 / p}(\Sigma)\right)\right) \times \mathbb{X}_{2}(J) \rightarrow L_{p}\left(J ; W_{p}^{2-1 / p}(\Sigma)\right)
$$

by $(B, u) \mapsto[t \mapsto B(t) u(t)]$. Since $T$ is real analytic, by (3.12), we get

$$
\left[(u,(\lambda, \mu)) \mapsto B_{\lambda, \mu}(u)\right] \in C^{\omega}\left(\mathbb{X}_{2} \times \mathbb{B}\left(0, r_{0}\right), L_{p}\left(J ; W_{p}^{2-1 / p}(\Sigma)\right)\right) .
$$

In virtue of Proposition 3.8(b) and the above bilinear map argument, one gets

$$
\Phi_{g} \in C^{\omega}\left(\mathbb{X}_{2} \times \mathbb{B}\left(0, r_{0}\right), L_{p}\left(J ; W_{p}^{2-1 / p}(\Sigma)\right) \times W_{p}^{4-3 / p}(\Sigma)\right) .
$$

By the implicit function theorem, there exists some $\mathbb{B}\left(0, r_{1}\right) \subset \mathbb{B}\left(0, r_{0}\right)$ such that

$$
\left[(\lambda, \mu) \mapsto \Theta_{\lambda, \mu}^{*} e^{\Delta_{\Sigma} t}\left(c+\Delta_{\Sigma}\right)^{-1} G\left(\hat{z}_{0}\right)\right] \in C^{\omega}\left(\mathbb{B}\left(0, r_{1}\right), \mathbb{X}_{2}\right)
$$

Without loss of generality, we may assume that $r_{1}=r_{0}$. In view of Proposition 3.8(b), we have that for all $s \in \mathbb{R}$

$$
\left[\mu \mapsto T_{\mu}\left(c+\Delta_{\Sigma}\right) T_{\mu}^{-1}\right] \in C^{\omega}\left(\mathbb{B}\left(0, r_{0}\right), C^{1}\left(J ; \mathcal{L}\left(W_{p}^{s+2}(\Sigma), W_{p}^{s}(\Sigma)\right)\right)\right) .
$$

The above bilinear map argument and the embedding $\mathbb{G}_{2}(J) \hookrightarrow \mathbb{F}_{2}(J)$ yield

$$
\left[(\lambda, \mu) \mapsto g_{1, \lambda, \mu}\right] \in C^{\omega}\left(\mathbb{B}\left(0, r_{0}\right), \mathbb{F}_{2}(J)\right)
$$

Following a similar discussion, one obtains

$$
\left[(\lambda, \mu) \mapsto q_{1, \lambda, \mu}\right] \in C^{\omega}\left(\mathbb{B}\left(0, r_{0}\right), \mathbb{F}_{3}(J)\right)
$$

(v) Regularity of the map $\Phi$. It follows from Proposition 3.6 that

$$
\left[(\lambda, \mu, \eta) \mapsto\left(\kappa_{A, \lambda, \mu, \eta}, d_{A, \lambda, \mu, \eta}\right)\right] \in C^{k}\left(\mathbb{B}\left(0, r_{0}\right), C^{1}(J ; B C(\Omega))^{2}\right) .
$$

Proposition 3.8 implies that

$$
\left[(\lambda, \mu) \mapsto\left(l_{A, \lambda, \mu}, d_{A, \lambda, \mu}\right)\right] \in C^{k}\left(\mathbb{B}\left(0, r_{0}\right), C^{1}\left(J ; B C^{1}(\Sigma)\right)^{2}\right) .
$$

Similarly, we have

$$
\left[(\lambda, \mu) \mapsto\left(l_{1, \lambda, \mu}, \gamma_{1, \lambda, \mu}\right)\right] \in C^{k}\left(\mathbb{B}\left(0, r_{0}\right),\left(C^{1}(J ; B C(\Sigma)) \cap C\left(J ; B C^{2}(\Sigma)\right)\right)^{2}\right) .
$$


By Proposition 3.6, one concludes that

$$
\left[(\mu, \eta) \mapsto T_{\mu, \eta} \Delta T_{\mu, \eta}^{-1}\right] \in C^{\omega}\left(\mathbb{B}\left(0, r_{0}\right), C^{1}\left(J ; \mathcal{L}\left(H_{p}^{2}(\Omega \backslash \Sigma), L_{p}(\Omega)\right)\right)\right) .
$$

Combined with (4.15), this yields

$$
\left[(\lambda, \mu, \eta) \mapsto d_{A, \lambda, \mu, \eta} T_{\mu, \eta} \Delta T_{\mu, \eta}^{-1}\right] \in C^{k}\left(\mathbb{B}\left(0, r_{0}\right), \mathcal{L}\left(\mathbb{E}_{1}(J), \mathbb{F}_{1}(J)\right)\right) .
$$

It follows again from (4.15) that

$$
\left[(\vartheta,(\lambda, \mu, \eta)) \mapsto \kappa_{A, \lambda, \mu, \eta} \partial_{t} \vartheta\right] \in C^{k}\left(\mathbb{E}_{1}(J) \times \mathbb{B}\left(0, r_{0}\right), \mathbb{F}_{1}(J)\right) .
$$

Together with Proposition 3.10, the above discussion shows that

$$
\begin{aligned}
{[(\vartheta,(\lambda, \mu, \eta))} & \left.\mapsto \kappa_{A, \lambda, \mu, \eta} \partial_{t} \vartheta-\left(1+\xi^{\prime} \lambda\right) d_{A, \lambda, \mu, \eta} T_{\mu, \eta} \Delta T_{\mu, \eta}^{-1} \vartheta-B_{\lambda, \mu, \eta}(\vartheta)\right] \\
& \in C^{k}\left(\mathbb{E}_{1}(J) \times \mathbb{B}\left(0, r_{0}\right), \mathbb{F}_{1}(J)\right) .
\end{aligned}
$$

Applying (4.13)-(4.17), one can check the regularity of all the other entries of $\Phi$, and thus

$$
\Phi \in C^{k}\left(\mathbb{E}(J) \times \mathbb{B}\left(0, r_{0}\right), \mathbb{F}(J)\right) .
$$

Now the implicit function theorem yields

$$
\left[(\lambda, \mu, \eta) \mapsto z_{\lambda, \mu, \eta}^{*}\right] \in C^{k}\left(\mathbb{B}\left(0, r_{0}\right), \mathbb{E}(J)\right) .
$$

As a conclusion of Theorem 3.12, we obtain

$$
z^{*} \in C^{k}\left(\stackrel{\circ}{J} \times \mathrm{T}_{a / 3} \backslash \Sigma\right) \times C^{k}(\stackrel{\circ}{J} \times \Sigma) .
$$

To attain the regularity of $\vartheta^{*}$ in $\Omega \backslash \overline{\mathrm{T}}_{a / 6}$, we study the linear equations

$$
\left\{\begin{aligned}
\kappa_{1}\left(\vartheta_{A}\right) \partial_{t} \vartheta-d_{1}\left(\vartheta_{A}\right) \Delta \vartheta & =0 & & \text { in } & & \Omega_{1} \backslash \overline{\mathrm{T}}_{a / 6} \\
\vartheta & =\vartheta^{*} & & \text { on } & & \partial_{1} \mathrm{~T}_{a / 6} \\
\vartheta(0) & =\hat{\vartheta}_{0} & & \text { in } & & \Omega_{1} \backslash \overline{\mathrm{T}}_{a / 6},
\end{aligned}\right.
$$

and

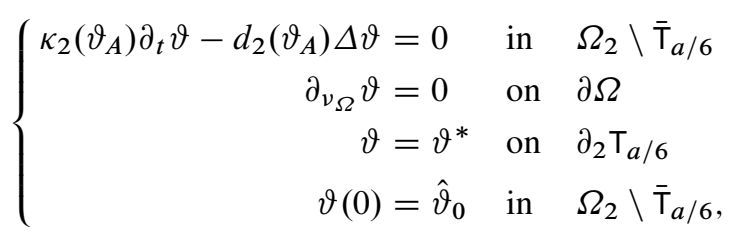

where $\partial_{i} \mathrm{~T}_{a / 6}:=\Lambda\left(\Sigma,(-1)^{i} a / 6\right)$. Since $\vartheta^{*}$ is analytic on $\partial_{i} \mathrm{~T}_{a / 6}$, we can obtain the regularity of $\vartheta^{*}$ in these two domains by means of the parameter-dependent diffeomorphism defined in [26], the results in [21, Section 8], and the implicit function theorem as above. To sum up, we have established the analyticity of the solution $z^{*}$, i.e.,

$$
z^{*} \in C^{k}(\stackrel{0}{J} \times \Omega \backslash \Sigma) \times C^{k}(\stackrel{0}{J} \times \Sigma) .
$$




\subsection{Reduction to zero initial data}

Recall that $\hat{z}=(\hat{\vartheta}, \hat{h})$ is the unique $L_{p}$ solution to problem (4.2) with initial data $\hat{z}_{0}$. We set $\bar{\vartheta}=\hat{\vartheta}-\vartheta^{*}, \bar{h}=\hat{h}-h^{*}$. Then $\bar{z}=(\bar{\vartheta}, \bar{h})$ is the unique solution to the following equation.

$$
\left\{\begin{aligned}
\kappa_{A} \partial_{t} \vartheta-d_{A} \Delta \vartheta & =F\left(\vartheta+\vartheta^{*}, h+h^{*}\right) & & \text { in } \quad \Omega \backslash \Sigma, \\
\partial_{\nu_{\Omega}} \vartheta & =0 & & \text { on } \quad \partial \Omega, \\
\llbracket \vartheta \rrbracket & =0 & & \text { on } \Sigma, \\
l_{1} \vartheta+\sigma_{0} \Delta_{\Sigma} h-\gamma_{1} \partial_{t} h & =\bar{G}\left(\vartheta, h ; \vartheta^{*}, h^{*}\right) & & \text { on } \Sigma, \\
l_{A} \partial_{t} h-\llbracket d_{A} \partial_{\nu} \vartheta \rrbracket & =\bar{Q}\left(\vartheta, h ; \vartheta^{*}, h^{*}\right) & & \text { on } \Sigma, \\
\vartheta(0) & =0 & & \text { in } \Omega \backslash \Sigma, \\
h(0) & =0 & & \text { on } \Sigma .
\end{aligned}\right.
$$

Here we have set

$$
\begin{aligned}
& \bar{G}\left(\vartheta, h ; \vartheta^{*}, h^{*}\right)=G\left(\vartheta+\vartheta^{*}, h+h^{*}\right)-e^{\Delta_{\Sigma^{t}}} G\left(\hat{z}_{0}\right), \\
& \bar{Q}\left(\vartheta, h ; \vartheta^{*}, h^{*}\right)=Q\left(\vartheta+\vartheta^{*}, h+h^{*}\right)-e^{\Delta_{\Sigma} t} Q\left(\hat{z}_{0}\right) .
\end{aligned}
$$

Note that $\bar{G}\left(0,0 ; \hat{\vartheta}_{0}, \hat{h}_{0}\right)=\bar{Q}\left(0,0 ; \hat{\vartheta}_{0}, \hat{h}_{0}\right)=0$ by construction, which ensures time trace zero at $t=0$. Let $z_{\lambda, \mu, \eta}^{*}:=\left(\vartheta_{\lambda, \mu, \eta}^{*}, h_{\lambda, \mu}^{*}\right)$. As in Section 5.1, we compute the temporal derivative of $\bar{z}_{\lambda, \mu, \eta}:=\left(\bar{\vartheta}_{\lambda, \mu, \eta}, \bar{h}_{\lambda, \mu}\right)$ as follows.

$$
\begin{array}{r}
\partial_{t}\left[\bar{\vartheta}_{\lambda, \mu, \eta}\right]=\left(1+\xi^{\prime} \lambda\right)\left(d_{A, \lambda, \mu, \eta} / \kappa_{A, \lambda, \mu, \eta}\right) T_{\mu, \eta} \Delta T_{\mu, \eta}^{-1} \bar{\vartheta}_{\lambda, \mu, \eta} \\
+B_{\lambda, \mu, \eta}\left(\bar{\vartheta}_{\lambda, \mu, \eta}\right) \\
+F_{\lambda, \mu, \eta}\left(\bar{z}_{\lambda, \mu, \eta}, z_{\lambda, \mu, \eta}^{*}\right) / \kappa_{A, \lambda, \mu, \eta},
\end{array}
$$

and either

$$
\partial_{t}\left[\bar{h}_{\lambda, \mu}\right]=\left(1+\xi^{\prime} \lambda\right) \llbracket\left(d_{A, \lambda, \mu} / l_{A, \lambda, \mu}\right) \partial_{\nu} \bar{\vartheta} \rrbracket+B_{\lambda, \mu}\left(\bar{h}_{\lambda, \mu}\right)+\bar{Q}_{\lambda, \mu}\left(\bar{z}_{\lambda, \mu, \eta}, z_{\lambda, \mu, \eta}^{*}\right) / l_{A, \lambda, \mu},
$$

when $\gamma \equiv 0$, or when $\gamma>0$ we have

$$
\begin{aligned}
\partial_{t}\left[\bar{h}_{\lambda, \mu}\right]=\left(1+\xi^{\prime} \lambda\right)\left(l_{1, \lambda, \mu} \bar{\vartheta}_{\lambda, \mu}+\sigma_{0} T_{\mu} \Delta_{\Sigma} T_{\mu}^{-1} \bar{h}_{\lambda, \mu}\right) / \gamma_{1, \lambda, \mu} & +B_{\lambda, \mu}\left(\bar{h}_{\lambda, \mu}\right) \\
& -\bar{G}_{\lambda, \mu}\left(\bar{z}_{\lambda, \mu, \eta}, z_{\lambda, \mu, \eta}^{*}\right) / \gamma_{1, \lambda, \mu}
\end{aligned}
$$

For sufficiently small $r_{0}, \Theta_{\mu, \eta}(z)=z$ for $(\mu, \eta) \in \mathbb{B}\left(0, r_{0}\right)$ and $z \in \Omega \backslash \mathrm{T}_{a / 3}$. Hence, $(\zeta \circ$ $\left.d_{\Sigma}\right)_{\lambda, \mu, \eta}=\zeta \circ d_{\Sigma}$. In virtue of $\left(\zeta \circ d_{\Sigma}\right)_{\lambda, \mu, \eta}(x)=0$ for $x \notin \Omega \backslash \mathrm{T}_{2 a / 3}$, and $(h \circ \Pi)_{\lambda, \mu, \eta}(x)=$ $\left(h_{\lambda, \mu} \circ \Pi\right)(x)$ for $x \in \mathrm{T}_{2 a / 3}$, one readily verifies that

$$
\begin{aligned}
\Theta_{\lambda, \mu, \eta}^{*} \Upsilon(h) & =\left(\zeta \circ d_{\Sigma}\right)_{\lambda, \mu, \eta}\left(h_{\lambda, \mu} \circ \Pi\right)_{\lambda, \mu, \eta}\left(v_{\Sigma} \circ \Pi\right)_{\lambda, \mu, \eta} \\
& =\left(\zeta \circ d_{\Sigma}\right)\left(h_{\lambda, \mu} \circ \Pi\right)\left(v_{\Sigma} \circ \Pi\right)_{\lambda, \mu, \eta}
\end{aligned}
$$


In the above expressions, for $z=(\vartheta, h)$,

$$
\begin{aligned}
F_{\lambda, \mu, \eta}\left(z, z_{\lambda, \mu, \eta}^{*}\right)=( & \left.+\xi^{\prime} \lambda\right) \Theta_{\lambda, \mu, \eta}^{*} F\left(\Theta_{*}^{\lambda, \mu, \eta}\left(z+z_{\lambda, \mu, \eta}^{*}\right)\right) \\
=( & \left.\kappa_{A, \lambda, \mu, \eta}-\kappa\left(\vartheta+\vartheta_{\lambda, \mu, \eta}^{*}\right)\right)\left[\partial_{t}\left(\vartheta+\vartheta_{\lambda, \mu, \eta}^{*}\right)-B_{\lambda, \mu, \eta}\left(\vartheta+\vartheta_{\lambda, \mu, \eta}^{*}\right)\right] \\
& +\left(1+\xi^{\prime} \lambda\right)\left\{\left(d\left(\vartheta+\vartheta_{\lambda, \mu, \eta}^{*}\right)-d_{A, \lambda, \mu, \eta}\right) T_{\mu, \eta} \Delta T_{\mu, \eta}^{-1}\left(\vartheta+\vartheta_{\lambda, \mu, \eta}^{*}\right)\right. \\
& -d\left(\vartheta+\vartheta_{\lambda, \mu, \eta}^{*}\right) M_{2, \lambda, \mu, \eta}\left(h+h_{\lambda, \mu}^{*}\right): T_{\mu, \eta} \nabla^{2} T_{\mu, \eta}^{-1}\left(\vartheta+\vartheta_{\lambda, \mu, \eta}^{*}\right) \\
& +d^{\prime}\left(\vartheta+\vartheta_{\lambda, \mu, \eta}^{*}\right)\left|\left(I-M_{1, \lambda, \mu, \eta}\left(h+h_{\lambda, \mu}^{*}\right)\right) T_{\mu, \eta} \nabla T_{\mu, \eta}^{-1}\left(\vartheta+\vartheta_{\lambda, \mu, \eta}^{*}\right)\right|^{2} \\
& -d\left(\vartheta+\vartheta_{\lambda, \mu, \eta}^{*}\right)\left(M_{3, \lambda, \mu, \eta}\left(h+h_{\lambda, \mu}^{*}\right) \mid T_{\mu, \eta} \nabla T_{\mu, \eta}^{-1}\left(\vartheta+\vartheta_{\lambda, \mu, \eta}^{*}\right)\right) \\
& \left.+\kappa\left(\vartheta+\vartheta_{\lambda, \mu, \eta}^{*}\right) \mathbb{R}_{\lambda, \mu, \eta}\left(h+h_{\lambda, \mu}^{*}\right)\left(\vartheta+\vartheta_{\lambda, \mu, \eta}^{*}\right)\right\},
\end{aligned}
$$

with

$$
\begin{aligned}
\Upsilon_{\lambda, \mu, \eta}(h)= & \Theta_{\lambda, \mu, \eta}^{*} \Upsilon\left(\Theta_{*}^{\lambda, \mu} h\right)=\left(\zeta \circ d_{\Sigma}\right)(h \circ \Pi)\left(v_{\Sigma} \circ \Pi\right)_{\lambda, \mu, \eta} \\
M_{1, \lambda, \mu, \eta}(h)= & \Theta_{\lambda, \mu, \eta}^{*} M_{1}\left(\Theta_{*}^{\lambda, \mu} h\right) \\
= & {\left[\left(I+\left(T_{\mu, \eta} \nabla T_{\mu, \eta}^{-1} \Upsilon_{\lambda, \mu, \eta}(h)\right)^{\top}\right)^{-1}\left(T_{\mu, \eta} \nabla T_{\mu, \eta}^{-1} \Upsilon_{\lambda, \mu, \eta}(h)\right)^{\top}\right]^{\top}, } \\
M_{2, \lambda, \mu, \eta}(h)= & \Theta_{\lambda, \mu, \eta}^{*} M_{2}\left(\Theta_{*}^{\lambda, \mu} h\right) \\
= & M_{1, \lambda, \mu, \eta}(h)+\left(M_{1, \lambda, \mu, \eta}(h)\right)^{\top}-M_{1, \lambda, \mu, \eta}(h)\left(M_{1, \lambda, \mu, \eta}(h)\right)^{\top}, \\
M_{3, \lambda, \mu, \eta}(h)= & \Theta_{\lambda, \mu, \eta}^{*} M_{3}\left(\Theta_{*}^{\lambda, \mu} h\right)=\left(I-M_{1, \lambda, \mu, \eta}(h)\right): T_{\mu, \eta} \nabla T_{\mu, \eta}^{-1} M_{1, \lambda, \mu, \eta}(h), \\
\mathbb{R}_{\lambda, \mu, \eta}(h) \vartheta= & \Theta_{\lambda, \mu, \eta}^{*} \mathbb{R}\left(\Theta_{*}^{\lambda, \mu} h\right) \Theta_{*}^{\lambda, \mu, \eta} \vartheta \\
= & \left(T_{\mu, \eta} \nabla T_{\mu, \eta}^{-1} \vartheta \mid\left(I+\left(T_{\mu, \eta} \nabla T_{\mu, \eta}^{-1} \Upsilon_{\lambda, \mu, \eta}(h)\right)^{\top}\right)^{-1}\right. \\
& {\left.\left[\partial_{t} \Upsilon_{\lambda, \mu, \eta}(h)-B_{\lambda, \mu, \eta}\left(\Upsilon_{\lambda, \mu, \eta}(h)\right)\right]\right) /\left(1+\xi^{\prime} \lambda\right), }
\end{aligned}
$$

and

$$
\begin{aligned}
\bar{G}_{\lambda, \mu}\left(z, z_{\lambda, \mu, \eta}^{*}\right)= & \left(1+\xi^{\prime} \lambda\right) \Theta_{\lambda, \mu}^{*} \bar{G}\left(\Theta_{*}^{\lambda, \mu}\left(z+z_{\lambda, \mu, \eta}^{*}\right)\right) \\
= & \left\{\gamma\left(\vartheta+\vartheta_{\lambda, \mu}^{*}\right) \beta_{\lambda, \mu}\left(h+h_{\lambda, \mu}^{*}\right)-\gamma_{1, \lambda, \mu}\right\}\left[\partial_{t}\left(h+h_{\lambda, \mu}^{*}\right)-B_{\lambda, \mu}\left(h+h_{\lambda, \mu}^{*}\right)\right] \\
& +\left(1+\xi^{\prime} \lambda\right)\left\{-\left(\llbracket \psi\left(\vartheta+\vartheta_{\lambda, \mu}^{*}\right) \rrbracket+\sigma \mathcal{H}_{\lambda, \mu}\left(h+h_{\lambda, \mu}^{*}\right)\right)+l_{1, \lambda, \mu}\left(\vartheta+\vartheta_{\lambda, \mu}^{*}\right)\right. \\
& \left.+\sigma_{0} T_{\mu} \Delta_{\Sigma} T_{\mu}^{-1}\left(h+h_{\lambda, \mu}^{*}\right)-g_{1, \lambda, \mu}\right\},
\end{aligned}
$$

with $\mathcal{H}_{\lambda, \mu}=\Theta_{\lambda, \mu}^{*} \mathcal{H} \Theta_{*}^{\lambda, \mu}$, and

$$
\begin{aligned}
M_{0, \lambda, \mu}(h) & =\Theta_{\lambda, \mu}^{*} M_{0}\left(\Theta_{*}^{\lambda, \mu} h\right)=\left(I-h L_{\Sigma, \lambda, \mu}\right)^{-1}, \\
\alpha_{\lambda, \mu}(h) & =\Theta_{\lambda, \mu}^{*} \alpha\left(\Theta_{*}^{\lambda, \mu} h\right)=M_{0, \lambda, \mu}(h) T_{\mu} \nabla T_{\mu}^{-1} h, \\
\beta_{\lambda, \mu}(h) & =\Theta_{\lambda, \mu}^{*} \beta\left(\Theta_{*}^{\lambda, \mu} h\right)=\left(1+\left|\alpha_{\lambda, \mu}(h)\right|^{2}\right)^{-1 / 2},
\end{aligned}
$$


and

$$
\begin{aligned}
\bar{Q}_{\lambda, \mu}\left(z, z_{\lambda, \mu, \eta}^{*}\right)= & \left(1+\xi^{\prime} \lambda\right) \Theta_{\lambda, \mu}^{*} \bar{Q}\left(\Theta_{*}^{\lambda, \mu}\left(z+z_{\lambda, \mu, \eta}^{*}\right)\right) \\
= & \gamma\left(\vartheta+\vartheta_{\lambda, \mu}^{*}\right) \beta_{\lambda, \mu}\left(h+h_{\lambda, \mu}^{*}\right)\left[\partial_{t}\left(h+h_{\lambda, \mu}^{*}\right)-B_{\lambda, \mu}\left(h+h_{\lambda, \mu}^{*}\right)\right]^{2} /\left(1+\xi^{\prime} \lambda\right) \\
& +\left(l_{A, \lambda, \mu}-l\left(\vartheta+\vartheta_{\lambda, \mu}^{*}\right)\right)\left[\partial_{t}\left(h+h_{\lambda, \mu}^{*}\right)-B_{\lambda, \mu}\left(h+h_{\lambda, \mu}^{*}\right)\right] \\
& +\left(1+\xi^{\prime} \lambda\right)\left\{\llbracket\left(d\left(v+\bar{\vartheta}_{\lambda, \mu}\right)-d_{A, \lambda, \mu}\right) \partial_{\nu}\left(\vartheta+\vartheta_{\lambda, \mu}^{*}\right) \rrbracket-q_{1, \lambda, \mu}\right. \\
& \left.\quad-\left(\llbracket d\left(\vartheta+\vartheta_{\lambda, \mu}^{*}\right) T_{\mu} \nabla T_{\mu}^{-1}\left(\vartheta+\vartheta_{\lambda, \mu}^{*}\right) \rrbracket \mid M_{4, \lambda, \mu}\left(h+h_{\lambda, \mu}^{*}\right) T_{\mu} \nabla_{\Sigma} T_{\mu}^{-1}\left(h+h_{\lambda, \mu}^{*}\right)\right)\right\}
\end{aligned}
$$

with $M_{4, \lambda, \mu}(h)=\Theta_{\lambda, \mu}^{*} M_{4}\left(\Theta_{*}^{\lambda, \mu} h\right)=\left(I-M_{1, \lambda, \mu}(h)\right)^{\top} M_{0, \lambda, \mu}(h)$.

Consider the map $\Phi:{ }_{0} \mathbb{E}(J) \rightarrow{ }_{0} \mathbb{F}(J):((\vartheta, h),(\lambda, \mu, \eta)) \mapsto$

$$
\begin{cases}\kappa_{A, \lambda, \mu, \eta} \partial_{t} \vartheta-\left(1+\xi^{\prime} \lambda\right) d_{A, \lambda, \mu, \eta} T_{\mu, \eta} \Delta T_{\mu, \eta}^{-1} \vartheta-\kappa_{A, \lambda, \mu, \eta} B_{\lambda, \mu, \eta}(\vartheta) & \\ \quad-F_{\lambda, \mu, \eta}\left(z, z_{\lambda, \mu, \eta}^{*}\right) & \text { in } \Omega \backslash \Sigma, \\ \left(1+\operatorname{sgn}(\gamma) \xi^{\prime} \lambda\right)\left(l_{1, \lambda, \mu} \vartheta+\sigma_{0} T_{\mu} \Delta_{\Sigma} T_{\mu}^{-1} h\right)-\gamma_{1, \lambda, \mu} \partial_{t} h & \\ \quad+\gamma_{1, \lambda, \mu} B_{\lambda, \mu}(h)-\bar{G}_{\lambda, \mu}\left(z, z_{\lambda, \mu, \eta}^{*}\right) & \text { on } \Sigma, \\ l_{A, \lambda, \mu} \partial_{t} h-\left(1+\xi^{\prime} \lambda\right)\left(\llbracket d_{A, \lambda, \mu} \partial_{\nu} \vartheta \rrbracket\right)-l_{A, \lambda, \mu} B_{\lambda, \mu}(h)-\bar{Q}_{\lambda, \mu}\left(z, z_{\lambda, \mu, \eta}^{*}\right) & \text { on } \Sigma,\end{cases}
$$

where $z=(\vartheta, h)$. Note that $\Phi\left(\bar{z}_{\lambda, \mu},(\lambda, \mu)\right)=0$ for all $(\lambda, \mu) \in \mathbb{B}\left(0, r_{0}\right)$. It is understood that ${ }_{0} \mathbb{F}(J):=\mathbb{F}_{1}(J) \times{ }_{0} \mathbb{F}_{2}(J) \times{ }_{0} \mathbb{F}_{3}(J)$.

(i) First, we shall check that $\Phi$ actually maps into ${ }_{0} \mathbb{F}(J)$. Functions in ${ }_{0} \mathbb{F}(J)$ automatically satisfy (LCe). One can check the temporal traces without difficulty. Indeed, recalling that $z_{\lambda, \mu, \eta}^{*}(0, \cdot)=$ $z^{*}(0, \cdot)$, we have

$$
\begin{aligned}
& \bar{G}_{\lambda, \mu}\left(z, z_{\lambda, \mu, \eta}^{*}\right)(0)=\Theta_{\lambda, \mu}^{*} G\left(z^{*}\right)(0)-g_{1}(0)=0, \\
& \bar{Q}_{\lambda, \mu}\left(z, z_{\lambda, \mu, \eta}^{*}\right)(0)=\Theta_{\lambda, \mu}^{*} Q\left(z^{*}\right)(0)-q_{1}(0)=0 .
\end{aligned}
$$

It suffices to check regularity of $F_{\lambda, \mu, \eta}\left(z, z_{\lambda, \mu, \eta}^{*}\right), \bar{G}_{\lambda, \mu}\left(z, z_{\lambda, \mu, \eta}^{*}\right)$ and $\bar{Q}_{\lambda, \mu}\left(z, z_{\lambda, \mu, \eta}^{*}\right)$, which will become clear in our argument for the regularity of $\Phi$.

It follows from well-known results for substitution operators for Sobolev spaces and (4.18) that

$$
\left[(\vartheta,(\lambda, \mu, \eta)) \mapsto \kappa\left(\vartheta+\vartheta_{\lambda, \mu, \eta}^{*}\right)\right] \in C^{k}\left({ }_{0} \mathbb{E}_{1}(J) \times \mathbb{B}\left(0, r_{0}\right), C(J, B C(\Omega))\right) .
$$

Analogous statements hold for $d\left(\vartheta+\vartheta_{\lambda, \mu, \eta}^{*}\right), d^{\prime}\left(\vartheta+\vartheta_{\lambda, \mu, \eta}^{*}\right), l\left(\vartheta+\vartheta_{\lambda, \mu, \eta}^{*}\right), \gamma\left(\vartheta+\vartheta_{\lambda, \mu, \eta}^{*}\right)$ and $\psi\left(\vartheta+\vartheta_{\lambda, \mu, \eta}^{*}\right)$ as well.

Adopting the notation in Section 2, we introduce an extension operator

$$
e_{\Pi}: \mathbb{E}_{2}(J) \rightarrow \mathbb{E}_{2}\left(J, \mathrm{~T}_{a}\right): \quad h \mapsto h \circ \Pi .
$$

$\left(\left(\pi_{\kappa} \circ \Pi\right)^{2}\right)_{\kappa \in \mathfrak{K}}$ forms a partition of unity for $\mathrm{T}_{a}$. For $\mathfrak{F} \in\left\{W_{p}, H_{p}\right\}$, we define

$$
\begin{aligned}
& \mathbb{R}_{\Pi}^{c}: \mathfrak{F}^{s}\left(\mathrm{~T}_{a}, E\right) \rightarrow \prod_{\kappa \in \mathfrak{K}} \mathfrak{F}^{s}\left(\mathrm{~T}_{a, \kappa}, E\right): u \mapsto\left(u \pi_{\kappa} \circ \Pi\right)_{\kappa}, \\
& \mathbb{R}_{\Pi}: \prod_{\kappa \in \mathfrak{K}} \mathfrak{F}^{s}\left(\mathrm{~T}_{a, \kappa}, E\right) \rightarrow \mathfrak{F}^{s}\left(\mathrm{~T}_{a}, E\right):\left(u_{\kappa}\right)_{\kappa} \mapsto \sum_{\kappa \in \mathfrak{K}} u_{\kappa} \pi_{\kappa} \circ \Pi .
\end{aligned}
$$


Then $\mathbb{R}_{\Pi}$ is a retraction with $\mathbb{R}_{\Pi}^{c}$ as a coretraction. By using this retraction-coretraction system, it is a simple matter to check that

$$
e_{\Pi} \in \mathcal{L}\left(\mathbb{E}_{2}(J), \mathbb{E}_{2}\left(J, \mathrm{~T}_{a}\right)\right)
$$

Extending $v:=\left(\zeta \circ d_{\Sigma}\right)\left(v_{\Sigma} \circ \Pi\right)$ to be identically zero outside $\mathrm{T}_{a}$, it belongs to $B C^{\infty}\left(\Omega, \mathbb{R}^{m+1}\right) \cap$ $C^{\omega}\left(\mathrm{T}_{a / 3}, \mathbb{R}^{m+1}\right)$. Proposition 3.6(a) implies

$$
\left[(\lambda, \mu, \eta) \mapsto v_{\lambda, \mu, \eta}\right] \in C^{\omega}\left(\mathbb{B}\left(0, r_{0}\right), C^{1}\left(J, B C^{k}\left(\Omega, \mathbb{R}^{m+1}\right)\right)\right)
$$

for all $k \in \mathbb{N}_{0}$. Proposition 3.8 and (4.18) then yield

$$
\left[(h,(\lambda, \mu)) \mapsto \Upsilon_{\lambda, \mu, \eta}\left(h+h_{\lambda, \mu}^{*}\right)\right] \in C^{\omega}\left({ }_{0} \mathbb{E}_{2}(J) \times \mathbb{B}\left(0, r_{0}\right), \mathbb{E}_{2}\left(J, \Omega ; \mathbb{R}^{m+1}\right)\right)
$$

Because $\mathbb{F}_{j}(J, \Omega)$ with $j=2,3$ and $W_{p}^{1-1 / 2 p}\left(J ; H_{p}^{1}(\Omega)\right) \cap L_{p}\left(J ; W_{p}^{3-1 / p}(\Omega)\right)$ are Banach algebras, we verify via Proposition 3.6(b) that

$$
\left[(h,(\lambda, \mu, \eta)) \mapsto M_{j, \lambda, \mu, \eta}\left(h+h_{\lambda, \mu}^{*}\right)\right] \in C^{\omega}\left({ }_{0} \mathbb{E}_{2}(J) \times \mathbb{B}\left(0, r_{0}\right), \mathbb{F}_{2}\left(J, \Omega ; \mathbb{R}^{(m+1)^{2}}\right)\right)
$$

for $j=1,2,3$. Proposition 3.6(b) and (4.18) lead to

$$
\left[(\vartheta,(\lambda, \mu, \eta)) \mapsto T_{\mu, \eta} \nabla T_{\mu, \eta}^{-1}\left(\vartheta+\vartheta_{\lambda, \mu, \eta}\right)\right] \in C^{\omega}\left({ }_{0} \mathbb{E}_{1}(J) \times \mathbb{B}\left(0, r_{0}\right), \mathbb{F}_{3}\left(J, \Omega_{i} ; \mathbb{R}^{m+1}\right)\right) .
$$

In virtue of Proposition 3.6(b) and (3.11), we get

$$
\left[(z,(\lambda, \mu, \eta)) \mapsto \mathbb{R}_{\lambda, \mu, \eta}\left(h+h_{\lambda, \mu}^{*}\right)\left(\vartheta+\vartheta_{\lambda, \mu, \eta}^{*}\right)\right] \in C^{\omega}\left({ }_{0} \mathbb{E}(J) \mathbb{B}\left(0, r_{0}\right), \mathbb{F}_{3}\left(J, \Omega_{i}\right)\right)
$$

Now it is immediate that

$$
\left[(z,(\lambda, \mu)) \mapsto F_{\lambda, \mu, \eta}\left(z, z_{\lambda, \mu}^{*}\right)\right] \in C^{k}\left({ }_{0} \mathbb{E}(J) \times \mathbb{B}\left(0, r_{0}\right), \mathbb{F}_{1}(J)\right)
$$

Since $\mathbb{C}_{2}(J)$ is a multiplication algebra, we have

$$
\left[(h,(\lambda, \mu)) \mapsto M_{0, \lambda, \mu}\left(h+h_{\lambda, \mu}^{*}\right)\right] \in C^{\omega}\left({ }_{0} \mathbb{E}_{2}(J) \times \mathbb{B}\left(0, r_{0}\right), \mathbb{C}_{2}\left(J ; T_{1}^{1} \Sigma\right)\right) .
$$

Proposition 3.8 and point-wise multiplier results on $\Sigma$ then imply that

$$
\left[(h,(\lambda, \mu)) \mapsto \alpha_{\lambda, \mu}\left(h+h_{\lambda, \mu}^{*}\right)\right] \in C^{\omega}\left({ }_{0} \mathbb{E}_{2}(J) \times \mathbb{B}\left(0, r_{0}\right), \mathbb{F}_{2}(J ; T \Sigma)\right) .
$$

Combined with the properties for substitution operators for Sobolev-Slobodeckii spaces, it yields

$$
\left[(h,(\lambda, \mu)) \mapsto \beta_{\lambda, \mu}\left(h+h_{\lambda, \mu}^{*}\right)\right] \in C^{\omega}\left({ }_{0} \mathbb{E}_{2}(J) \times \mathbb{B}\left(0, r_{0}\right), \mathbb{F}_{2}(J)\right) .
$$

It is shown in [72] that in every coordinate patch $\left(\mathrm{O}_{\kappa}, \varphi_{\kappa}\right)$, the local expression of the mean curvature operator reads as

$$
(\mathcal{H}(h))_{\kappa}=\beta(h) \frac{P_{\kappa}\left(h, \partial_{j} h, \partial_{j k} h\right)}{R_{\kappa}\left(h, \partial_{j} h\right)} .
$$


Here $P_{\kappa}$ is a polynomial in $h$ and its derivatives up to second order, and $R_{\kappa}$ is a polynomial in $h$ and its first order derivatives. Both have $B C^{\infty} \cap C^{\omega}$-coefficients. We again use the fact that $\mathbb{F}_{2}(J)$ is a Banach algebra. Following a similar argument in the same reference, we get

$$
\left[(h,(\lambda, \mu)) \mapsto \mathcal{H}_{\lambda, \mu}\left(h+h_{\lambda, \mu}^{*}\right)\right] \in C^{\omega}\left({ }_{0} \mathbb{E}_{2}(J) \times \mathbb{B}\left(0, r_{0}\right), \mathbb{F}_{2}(J)\right) .
$$

By (4.20) and trace theorems of anisotropic Sobolev-Slobodeckii spaces, we infer that

$$
\left[\left.(h,(\lambda, \mu)) \mapsto M_{1, \lambda, \mu}\left(h+h_{\lambda, \mu}^{*}\right)\right|_{\Sigma}\right] \in C^{\omega}\left({ }_{0} \mathbb{E}_{2}(J) \times \mathbb{B}\left(0, r_{0}\right), \mathbb{F}_{3}\left(J ; \mathbb{R}^{(m+1)^{2}}\right) .\right.
$$

It follows from the point-wise multiplier theorem in [5, Section 9] that

$$
\left[(h,(\lambda, \mu)) \mapsto M_{4, \lambda, \mu}\left(h+h_{\lambda, \mu}^{*}\right)\right] \in C^{\omega}\left({ }_{0} \mathbb{E}_{2}(J) \times \mathbb{B}\left(0, r_{0}\right), \mathbb{F}_{3}\left(J ; \mathbb{R}^{(m+1)^{2}}\right)\right.
$$

To sum up, we conclude that

$$
\begin{aligned}
& {\left[(z,(\lambda, \mu)) \mapsto \bar{G}_{\lambda, \mu}\left(z, z_{\lambda, \mu}^{*}\right)\right] \in C^{k}\left({ }_{0} \mathbb{E}(J) \times \mathbb{B}\left(0, r_{0}\right), \mathbb{F}_{2}(J)\right),} \\
& {\left[(z,(\lambda, \mu)) \mapsto \bar{Q}_{\lambda, \mu}\left(z, z_{\lambda, \mu}^{*}\right)\right] \in C^{k}\left({ }_{0} \mathbb{E}(J) \times \mathbb{B}\left(0, r_{0}\right), \mathbb{F}_{3}(J)\right) .}
\end{aligned}
$$

Taking into account all the above discussion, this leads to

$$
\Phi \in C^{k}\left({ }_{0} \mathbb{E}(J) \times \mathbb{B}\left(0, r_{0}\right),{ }_{0} \mathbb{F}(J)\right) .
$$

(ii) We look at the Fréchet derivative of $\Phi$ with respect to ${ }_{0} \mathbb{E}(J)$ at $(\bar{z}, 0)$. To this end, we find it more convenient to consider the following non-linear maps.

$$
\begin{aligned}
& \hat{F}(z)=\left(\hat{\kappa}_{0}-\kappa(\vartheta)\right) \partial_{t} \vartheta+\left(d(\vartheta)-\hat{d}_{0}\right) \Delta \vartheta-d(\vartheta) M_{2}(h): \nabla^{2} \vartheta \\
&+d^{\prime}(\vartheta)\left|\left(I-M_{1}(h)\right) \nabla \vartheta\right|^{2}-d(\vartheta)\left(M_{3}(h) \mid \nabla \vartheta\right)+\kappa(\vartheta) R(h) \vartheta, \\
& \hat{G}(z)=-(\llbracket \psi(\vartheta) \rrbracket+\sigma \mathcal{H}(h))+\hat{l}_{1} \vartheta+\sigma_{0} \Delta_{\Sigma} h+\left(\gamma(\vartheta) \beta(h)-\hat{\gamma}_{1}\right) \partial_{t} h, \\
& \hat{Q}(z)=\llbracket\left(d(\vartheta)-\hat{d}_{0}\right) \partial_{\nu} \vartheta \rrbracket+\left(\hat{l}_{0}-l(\vartheta)\right) \partial_{t} h-\left(\llbracket d(\vartheta) \nabla \vartheta \rrbracket \mid M_{4}(h) \nabla_{\Sigma} h\right) \\
&+\gamma(v) \beta(h)\left(\partial_{t} h\right)^{2},
\end{aligned}
$$

i.e., replacing $\vartheta_{A}$ by $\hat{\vartheta}_{0}$ in the definition of $F, G$ and $H$. With only slight modification of (i), we immediately obtain

$$
(\hat{F}, \hat{G}, \hat{Q}) \in C^{k}\left(\mathbb{E}(J), \mathbb{F}_{1}(J) \times \mathbb{F}_{2}(J) \times \mathbb{F}_{3}(J)\right) .
$$

Recall that $\hat{z}=\bar{z}+z^{*}$. Letting $w=(u, \rho)$, one computes

$$
D_{1} \Phi(\bar{z}, 0) w= \begin{cases}\hat{\kappa}_{0} \partial_{t} u-\hat{d}_{0} \Delta u-\hat{F}^{\prime}(\hat{z}) w & \text { in } \quad \Omega \backslash \Sigma, \\ \hat{l}_{1} u+\sigma_{0} \Delta_{\Sigma} \rho-\hat{\gamma}_{1} \partial_{t} \rho-\hat{G}^{\prime}(\hat{z}) w & \text { on } \quad \Sigma, \\ \hat{l}_{0} \partial_{t} \rho-\llbracket \hat{d}_{0} \partial_{\nu} u \rrbracket-\hat{Q}^{\prime}(\hat{z}) w & \text { on } \quad \Sigma .\end{cases}
$$

A moment of reflection shows that we have the liberty to exchange the coefficients $\left(\kappa_{A}, d_{A}, l_{A}\right)$, used in the definition of $\Phi$, by $\left(\hat{\kappa}_{0}, \hat{d}_{0}, \hat{l}_{0}\right)$. These quantities are only used for the principal linearization, and are then subtracted off in the nonlinearities. 
We split $D_{1} \Phi(\bar{z}, 0)$ into two parts. Let $\hat{\beta}_{0}:=\beta\left(\hat{h}_{0}\right)$. Define $\mathbb{L}\left(\hat{\vartheta}_{0}\right):{ }_{0} \mathbb{E}(J) \rightarrow{ }_{0} \mathbb{F}(J)$ by

$$
\mathbb{L}\left(\hat{\vartheta}_{0}\right) w= \begin{cases}\hat{\kappa}_{0} \partial_{t} u-\hat{d}_{0}\left(I-M_{2}\left(\hat{h}_{0}\right)\right): \nabla^{2} u & \text { in } \quad \Omega \backslash \Sigma, \\ \hat{l}_{1} u+\sigma \mathcal{H}^{\prime}\left(\hat{h}_{0}\right) \rho-\hat{\gamma}_{1} \beta^{\prime}\left(\hat{h}_{0}\right) e^{\Delta_{\Sigma} t}\left(\partial_{t} \hat{h}(0)\right) \rho & \\ \quad-\hat{\gamma}_{1} \hat{\beta}_{0} \partial_{t} \rho-\gamma^{\prime}\left(e^{\Delta_{\Sigma} t} \hat{\vartheta}_{0}\right) \hat{\beta}_{0} e^{\Delta_{\Sigma} t}\left(\partial_{t} \hat{h}(0)\right) u & \text { on } \quad \Sigma, \\ \hat{l}_{0} \partial_{t} \rho-\left(\llbracket \hat{d}_{0} \nabla u \rrbracket \mid v_{\Sigma}-M_{4}\left(\hat{h}_{0}\right) \nabla_{\Sigma} \hat{h}_{0}\right) & \text { on } \quad \Sigma .\end{cases}
$$

and $\mathbb{K}(\hat{z}):{ }_{0} \mathbb{E}(J) \rightarrow{ }_{0} \mathbb{F}(J)$ by

$$
\mathbb{K}(\hat{z})=:\left(\mathbb{K}_{1}, \mathbb{K}_{2}, \mathbb{K}_{3}\right)^{\top}:=\mathbb{L}\left(\hat{\vartheta}_{0}\right) w-D_{1} \Phi(\hat{z}, 0) .
$$

TheOREM 4.2 Let $J=[0, T]$. Then $\mathbb{L}\left(\hat{\vartheta}_{0}\right) \in \operatorname{Lis}(\mathbb{E}(J), \mathbb{F}(J))$. In particular, $\mathbb{L}\left(\hat{\vartheta}_{0}\right) \in$ $\operatorname{Lis}\left({ }_{0} \mathbb{E}(J),{ }_{0} \mathbb{F}(J)\right)$.

Proof. We first solve the following model problem for the case $\gamma \equiv 0$. Suppose that

$$
\kappa_{0} \in B U C\left(\mathbb{R}^{m} \times \mathbb{R}_{+}\right), \quad d_{0} \in B U C^{1}\left(\mathbb{R}^{m}\right), \quad a_{0} \in B U C^{1}\left(\mathbb{R}^{m} ; \mathbb{R}^{m}\right), \quad \kappa_{0}, d_{0}>0 .
$$

Moreover, $l_{0} \in W_{p}^{2-6 / p}\left(\mathbb{R}^{m}\right), l_{2} \in \mathbb{F}_{2}\left(J, \mathbb{R}^{m}\right)$ satisfy $l_{2} l_{0}>0$. The differential operators $-P(x, y): \nabla^{2}$ and $-S(x): \nabla_{x}^{2}$ are uniformly elliptic. For any given $\left(f, g, q,\left(u_{0}, \rho_{0}\right)\right) \in$ $\mathbb{F}\left(J, \mathbb{R}^{m} \times \mathbb{R}_{+}\right)$,

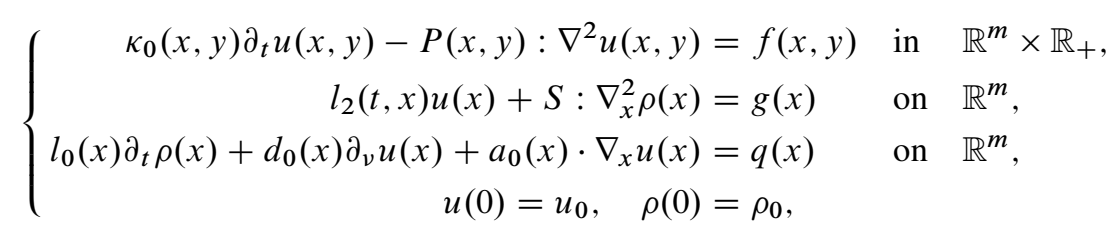

admits a unique solution $(u, \rho) \in \mathbb{E}\left(J, \mathbb{R}^{m} \times \mathbb{R}_{+}\right)$. We identify $\mathbb{R}^{m}$ with $\mathbb{R}^{m} \times\{0\}$. Here $x \in$ $\mathbb{R}^{m}, y \in \mathbb{R}_{+}$, and the space $\mathbb{F}\left(J, \mathbb{R}^{m} \times \mathbb{R}_{+}\right)$is defined by replacing $\Omega \backslash \Sigma$ and $\Sigma$ by $\mathbb{R}^{m} \times \mathbb{R}_{+}$and $\mathbb{R}^{m}$, respectively. The space $\mathbb{E}\left(J, \mathbb{R}^{m} \times \mathbb{R}_{+}\right)$is defined analogously. Similar problems have been considered in [22]. It suffices to check the Lopatinskii-Shapiro condition (LS) and the asymptotic Lopatinskii-Shapiro condition $\left(\mathbf{L S}_{\infty}^{+}\right)$defined therein. For simplicity, we assume all the coefficients to be constants and set

$$
P(\xi):=\xi_{e}^{\top} P \xi_{e}, \quad S(\xi):=\xi^{\top} S \xi, \quad P_{m+1}:=P_{m+1, m+1}
$$

and $P_{m}:=\left(P_{m+1,1}, \cdots, P_{m+1, m}\right)$ with $\xi \in \mathbb{R}^{m}$ and $\xi_{e}:=\left(\xi^{\top}, 0\right)^{\top} \in \mathbb{R}^{m+1}$. (LS) is satisfied, if for any $\xi \in \mathbb{R}^{m}$ and $\lambda \in \overline{\mathbb{C}}_{+}$with $|\xi|+|\lambda| \neq 0$, the following ordinary differential equation in $\mathbb{R}_{+}$

$$
\left\{\begin{aligned}
\left(\kappa_{0} \lambda+P(\xi)+2 i P_{m} \cdot \xi \partial_{y}-P_{m+1} \partial_{y}^{2}\right) v(y) & =0, \quad y>0, \\
l_{2} v(0)-S(\xi) \delta & =0 \\
l_{0} \lambda \delta-\left(i a_{0} \cdot \xi+d_{0}(x) \partial_{y}\right) v(0) & =0
\end{aligned}\right.
$$

has a unique solution $(v, \delta) \in C_{0}\left(\mathbb{R}_{+} ; \mathbb{C}\right) \times \mathbb{C}$. It is clear that the only stable solution to the first line is

$$
v(y)=e^{\mu y} v(0), \quad \mu:=\frac{\sqrt{-\left(P_{m} \cdot \xi\right)^{2}+P_{m+1} \kappa_{0} \lambda+P_{m+1} P(\xi)}+i P_{m} \cdot \xi}{P_{m+1}}
$$


in the case $\lambda \notin \mathbb{R}$, or, $v \equiv 0$, otherwise. We conclude from the second and third lines that

$$
\left[i S(\xi) a_{0} \cdot \xi+S(\xi) \mu d_{0}-l_{2} l_{0} \lambda\right] \delta=0
$$

It implies that $\delta=0, v \equiv 0$. $\left(\mathbf{L S}_{\infty}^{+}\right)$is satisfied, if for any $\xi \in \mathbb{R}^{m}$ and $\lambda \in \overline{\mathbb{C}}_{+}$with $|\xi|+|\lambda| \neq 0$,

$$
\left\{\begin{aligned}
\left(\kappa_{0} \lambda+P(\xi)+2 i P_{m} \cdot \xi \partial_{y}-P_{m+1} \partial_{y}^{2}\right) v(y) & =0, \quad y>0 \\
l_{2} v(0)-S(\xi) \delta & =0 \\
\left(i a_{0} \cdot \xi+d_{0}(x) \partial_{y}\right) v(0) & =0
\end{aligned}\right.
$$

and $|\xi|=1, \lambda \in \overline{\mathbb{C}}_{+} \backslash\{0\}$

$$
\left\{\begin{aligned}
\left(\kappa_{0} \lambda-P_{m+1} \partial_{y}^{2}\right) v(y) & =0, \quad y>0 \\
l_{2} v(0)-S(\xi) \delta & =0 \\
l_{0} \lambda \delta+d_{0}(x) \partial_{y} v(0) & =0
\end{aligned}\right.
$$

and $|\xi|=1, \lambda \in \overline{\mathbb{C}}_{+} \backslash\{0\}$

$$
\left\{\begin{aligned}
\left(\kappa_{0} \lambda-P_{m+1} \partial_{y}^{2}\right) v(y) & =0, \quad y>0 \\
l_{2} v(0)-S(\xi) \delta & =0 \\
d_{0}(x) \partial_{y} v(0) & =0
\end{aligned}\right.
$$

admit unique solutions $(v, \delta) \in C_{0}\left(\mathbb{R}_{+} ; \mathbb{C}\right) \times \mathbb{C}$. One can check in an analogous manner that stable solutions to those equations are trivial. For general coefficients, the problem can be proved by a perturbation argument as in [21]. The rest of the proof now follows from similar arguments to those of $[65$, Theorems 3.3, 3.5].

In order to prove that $D_{1} \Phi(\bar{z}, 0)$ is an isomorphism, we need to control the norm $\left\|\mathbb{K}\left(\hat{z}_{0}\right)\right\|_{\mathcal{L}\left({ }_{0} \mathbb{E}(J),_{0} \mathbb{F}(J)\right)}$. To this end, we first compute several derivatives related to $\mathbb{K}$ explicitly.

$$
\begin{aligned}
\hat{F}^{\prime}(z) w= & \left.\hat{\kappa}_{0}-\kappa(\vartheta)\right) \partial_{t} u-\kappa^{\prime}(\vartheta) u \partial_{t} \vartheta+\left(d(\vartheta)-\hat{d}_{0}\right) \Delta u+d^{\prime}(\vartheta) u \Delta \vartheta \\
& -d^{\prime}(\vartheta) u M_{2}(h): \nabla^{2} \vartheta-d(\vartheta) M_{2}^{\prime}(h) \rho: \nabla^{2} \vartheta-d(\vartheta) M_{2}(h): \nabla^{2} u \\
& -d^{\prime}(\vartheta) u\left(M_{3}(h) \mid \nabla \vartheta\right)-d(\vartheta)\left(M_{3}^{\prime}(h) \rho \mid \nabla \vartheta\right)-d(\vartheta)\left(M_{3}(h) \mid \nabla u\right) \\
& +2 d^{\prime}(\vartheta)\left(I-M_{1}(h) \nabla \vartheta \mid\left(I-M_{1}(h)\right) \nabla u-M_{1}^{\prime}(h) \rho \nabla \vartheta\right) \\
& +d^{\prime \prime}(\vartheta) u\left|\left(I-M_{1}(h)\right) \nabla \vartheta\right|^{2}+\kappa(\vartheta)\left(\nabla u \mid\left(I+\nabla \Upsilon(h)^{\top}\right)^{-1} \partial_{t} \Upsilon(h)\right) \\
& -\kappa(\vartheta)\left(\nabla \vartheta \mid\left(I+\nabla \Upsilon(h)^{\top}\right)^{-1} \nabla \Upsilon(\rho)^{\top}\left(I+\nabla \Upsilon(h)^{\top}\right)^{-1} \partial_{t} \Upsilon(h)\right) \\
& +\kappa(\vartheta)\left(\nabla \vartheta \mid\left(I+\nabla \Upsilon(h)^{\top}\right)^{-1} \partial_{t} \Upsilon(\rho)\right)+\kappa^{\prime}(\vartheta) u R(h) \vartheta,
\end{aligned}
$$

and

$$
\begin{aligned}
\hat{G}^{\prime}(z) w=-\left(\llbracket \psi^{\prime}(\vartheta) \rrbracket u+\sigma \mathcal{H}^{\prime}(h) \rho\right)+\hat{l}_{1} u+\sigma_{0} \Delta_{\Sigma} \rho+ & \left(\gamma(\vartheta) \beta(h)-\hat{\gamma}_{1}\right) \partial_{t} \rho \\
& +\left(\gamma^{\prime}(\vartheta) u \beta(h)-\gamma(\vartheta) \beta^{\prime}(h) \rho\right) \partial_{t} h,
\end{aligned}
$$


and

$$
\begin{aligned}
\hat{Q}^{\prime}(z) w= & \llbracket d^{\prime}(\vartheta) \partial_{v} \vartheta \rrbracket u+\llbracket\left(d(\vartheta)-\hat{d}_{0}\right) \partial_{v} u \rrbracket+\left(\hat{l}_{0}-l(\vartheta)\right) \partial_{t} \rho-l^{\prime}(\vartheta) u \partial_{t} h \\
& -\left(\llbracket d^{\prime}(\vartheta) \nabla \vartheta \rrbracket u \mid M_{4}(h) \nabla_{\Sigma} h\right)-\left(\llbracket d(\vartheta) \nabla u \rrbracket \mid M_{4}(h) \nabla_{\Sigma} h\right) \\
& -\left(\llbracket d(\vartheta) \nabla \vartheta \rrbracket \mid M_{4}(h) \nabla_{\Sigma} \rho\right)-\left(\llbracket d(\vartheta) \nabla \vartheta \rrbracket \mid M_{4}^{\prime}(h) \rho \nabla_{\Sigma} h\right) \\
& +\gamma^{\prime}(\vartheta) u \beta(h)\left[\partial_{t} h\right]^{2}+\gamma(\vartheta) \beta^{\prime}(h) \rho\left[\partial_{t} h\right]^{2}+2 \gamma(\vartheta) \beta(h) \partial_{t} h \partial_{t} \rho .
\end{aligned}
$$

The derivatives of $M_{0}(h), \alpha(h), \beta(h)$ and $\mathcal{H}(h)$ are given by

$$
\begin{aligned}
& M_{0}^{\prime}(h) \rho=\rho M_{0}(h) L_{\Sigma} M_{0}(h), \quad \alpha^{\prime}(h) \rho=M_{0}(h) \nabla_{\Sigma} \rho+\rho M_{0}(h) L_{\Sigma} M_{0}(h) \alpha(h), \\
& \beta^{\prime}(h) \rho=-\beta^{3}(h)\left(\alpha(h) \mid M_{0}(h) \nabla_{\Sigma} \rho+\rho M_{0}(h) L_{\Sigma} \alpha(h)\right), \\
& \mathcal{H}^{\prime}(h) \rho=\beta(h)\left\{\operatorname{tr}\left[M_{0}^{\prime}(h) \rho\left(L_{\Sigma}+\nabla_{\Sigma} \alpha(h)\right)\right]+\operatorname{tr}\left[M_{0}(h) \nabla_{\Sigma} \alpha^{\prime}(h) \rho\right]\right. \\
& \quad-2 \beta(h) \beta^{\prime}(h) \rho\left(M_{0}(h) \alpha(h) \mid\left[\nabla_{\Sigma} \alpha(h)\right] \alpha(h)\right)-\beta^{2}(h)\left(M_{0}^{\prime}(h) \rho \alpha(h) \mid\left[\nabla_{\Sigma} \alpha(h)\right] \alpha(h)\right) \\
& \quad-\beta^{2}(h)\left(M_{0}(h) \alpha^{\prime}(h) \rho \mid\left[\nabla_{\Sigma} \alpha(h)\right] \alpha(h)\right)-\beta^{2}(h)\left(M_{0}(h) \alpha(h) \mid\left[\nabla_{\Sigma} \alpha^{\prime}(h) \rho\right] \alpha(h)\right) \\
& \left.\quad-\beta^{2}(h)\left(M_{0}(h) \alpha(h) \mid\left[\nabla_{\Sigma} \alpha(h)\right] \alpha^{\prime}(h) \rho\right)\right\} / m+\beta^{\prime}(h) \rho(\mathcal{H}(h) / \beta(h)) .
\end{aligned}
$$

See [63, formula (32)] for a justification for the last equality.

We will use the following lemma frequently in the sequel.

Lemma 4.3 There exists a constant $C_{0}$ independent of $T$ such that

(a) For all $\left(v_{1}, v_{2}\right) \in \mathbb{F}_{j}(J) \times{ }_{0} \mathbb{F}_{j}(J)$ and $j=2,3$,

$$
\left\|v_{1} v_{2}\right\|_{\mathbb{F}_{j}(J)} \leqslant C_{0}\left(\left\|v_{1}\right\|_{C(J \times \Sigma)}+\left\|v_{1}\right\|_{\mathbb{F}_{j}(J)}\right)\left\|v_{2}\right\|_{\mathbb{F}_{j}(J)} .
$$

(b) For all $\left(v_{1}, v_{2}\right) \in{ }_{0} \mathbb{F}_{j}(J) \times{ }_{0} \mathbb{F}_{j}(J)$ and $j=2,3$,

$$
\left\|v_{1} v_{2}\right\|_{0^{0} \mathbb{F}_{j}(J)} \leqslant C_{0}\left\|v_{1}\right\|_{0} \mathbb{F}_{j}(J)\left\|v_{2}\right\|_{0} \mathbb{F}_{j}(J) .
$$

(c) For all $\left(v_{1}, v_{2}\right) \in \mathbb{F}_{3}(J) \times{ }_{0} \mathbb{F}_{2}(J)$

$$
\left\|v_{1} v_{2}\right\|_{\mathbb{F}_{3}(J)} \leqslant C_{0}\left\|v_{1}\right\|_{\mathbb{F}_{3}(J)}\left\|v_{2}\right\|_{0} \mathbb{F}_{2}(J) .
$$

Proof. (a) The case $j=2$ is shown in [60, Lemma 5.5(b)]. For the reader's convenience, we will nevertheless include a proof herein. The case $j=3$ follows in a similar way. By the retractioncoretraction system defined in Section 2, it suffices to show the estimates for functions on $Q^{m}$, i.e., we assume $\left(v_{1}, v_{2}\right) \in \mathbb{F}_{j}\left(J, \mathrm{Q}^{m}\right) \times{ }_{0} \mathbb{F}_{j}\left(J, \mathrm{Q}^{m}\right)$. We equip $\mathbb{F}_{2}\left(J, \mathrm{Q}^{m}\right)$ with the norm:

$$
\begin{aligned}
& \|v\|_{\mathbb{F}_{2}\left(J, \mathrm{Q}^{m}\right)}=\|v\|_{W_{p}^{1-1 / 2 p}\left(J ; L_{p}\left(\mathrm{Q}^{m}\right)\right)}+\|v\|_{L_{p}\left(J ; W_{p}^{2-1 / p}\left(\mathrm{Q}^{m}\right)\right)}, \\
& \|v\|_{L_{p}\left(J ; W_{p}^{2-1 / p}\left(\mathrm{Q}^{m}\right)\right)}=\|v\|_{L_{p}\left(J ; H_{p}^{1}\left(\mathrm{Q}^{m}\right)\right)}+\sum_{j=1}^{m}\left\|\left\langle\partial_{j} v\right\rangle_{W_{p}^{1-1 / p}\left(\mathrm{Q}^{m}\right)}\right\|_{L_{p}(J)} .
\end{aligned}
$$

Here $\langle\cdot\rangle_{W_{p}^{1-1 / p}(J ; X)}$ is the Slobodeckii seminorm of the space $W_{p}^{1-1 / p}(J ; X)$ for a Banach space 
$X$. Then,

$$
\begin{aligned}
& \left\|v_{1} v_{2}\right\|_{W_{p}^{1-1 / 2 p}\left(J ; L_{p}\left(\mathrm{Q}^{m}\right)\right)} \leqslant C^{\prime}\left\{\left\|v_{1}\right\|_{W_{p}^{1-1 / 2 p}\left(J ; L_{p}\left(\mathrm{Q}^{m}\right)\right)}\left\|v_{2}\right\|_{B C\left(J \times \mathrm{Q}^{m}\right)}\right. \\
& \left.\quad+\left(\int_{J} \int_{J}\left\|v_{1}(s)\left(v_{2}(t)-v_{2}(s)\right)\right\|_{L_{p}\left(\mathrm{Q}^{m}\right)}^{p} \frac{1}{|t-s|^{1 / 2+p}} d t d s\right)^{1 / p}\right\} \\
& \leqslant C^{\prime}\left\{\left\|v_{1}\right\|_{W_{p}^{1-1 / 2 p}\left(J ; L_{p}\left(\mathrm{Q}^{m}\right)\right)}\left\|v_{2}\right\|_{B C\left(J \times \mathrm{Q}^{m}\right)}+\left\|v_{1}\right\|_{B C\left(J \times \mathrm{Q}^{m}\right)}\left\langle v_{2}\right\rangle_{W_{p}^{1-1 / 2 p}\left(J ; L_{p}\left(\mathrm{Q}^{m}\right)\right)}\right\} .
\end{aligned}
$$

Similarly, one computes

$$
\begin{aligned}
& \left\|v_{1} v_{2}\right\|_{L_{p}\left(J ; W_{p}^{2-1 / p}\left(\mathrm{Q}^{m}\right)\right)} \leqslant C^{\prime}\left\{\left\|v_{1}\right\|_{L_{p}\left(J ; W_{p}^{2-1 / p}\left(\mathrm{Q}^{m}\right)\right)}\left\|v_{2}\right\|_{C\left(J, B C^{1}\left(\mathrm{Q}^{m}\right)\right)}\right. \\
& +\sum_{j=1}^{m}\left[\int_{J}\left(\int_{\mathrm{Q}^{m}} \int_{\mathrm{Q}^{m}} \mid v_{1}(t, x)\left(\partial_{j} v_{2}(t, x)-\left.\partial_{j} v_{2}(t, y)\right|^{p} \frac{1}{|x-y|^{m-1+p}} d x d y\right) d t\right]^{1 / p}\right. \\
& +\sum_{j=1}^{m}\left[\int_{J}\left(\int_{\mathrm{Q}^{m}} \int_{\mathrm{Q}^{m}} \mid \partial_{j} v_{1}(t, x)\left(v_{2}(t, x)-\left.v_{2}(t, y)\right|^{p} \frac{1}{|x-y|^{m-1+p}} d x d y\right) d t\right]^{1 / p}\right\} .
\end{aligned}
$$

We immediately have

$$
\begin{gathered}
\int_{J}\left(\int_{\mathrm{Q}^{m}} \int_{\mathrm{Q}^{m}} \mid v_{1}(t, x)\left(\partial_{j} v_{2}(t, x)-\left.\partial_{j} v_{2}(t, y)\right|^{p} \frac{1}{|x-y|^{m-1+p}} d x d y\right) d t\right. \\
\leqslant\left\|v_{1}\right\|_{B C\left(J \times \mathrm{Q}^{m}\right)}^{p}\left\|v_{2}\right\|_{L_{p}\left(J, W_{p}^{2-1 / p}\left(\mathrm{Q}^{m}\right)\right)}^{p} .
\end{gathered}
$$

The remaining estimate can be carried out as follows.

$$
\begin{aligned}
\int_{\mathrm{Q}^{m}} & \int_{\mathrm{Q}^{m}} \mid \partial_{j} v_{1}(t, x)\left(v_{2}(t, x)-\left.v_{2}(t, y)\right|^{p} \frac{1}{|x-y|^{m-1+p}} d x d y\right. \\
& \leqslant \int_{\mathrm{Q}^{m}} \int_{\mathrm{Q}^{m}}\left|\partial_{j} v_{1}(t, x)\right|^{p}\left(\int_{0}^{1}\left|\left(\nabla v_{2}(t, x+\tau(y-x)) \mid(y-x)\right)\right| d \tau\right)^{p} \frac{1}{|x-y|^{m-1+p}} d x d y \\
& \leqslant C^{\prime} \int_{\mathrm{Q}^{m}}\left|\partial_{j} v_{1}(t, x)\right|^{p} \int_{\mathrm{Q}^{m}} \frac{|x-y|^{p}}{|x-y|^{m-1+p}} d y d x\left\|\nabla v_{2}(t)\right\|_{B C\left(\mathrm{Q}^{m}\right)}^{p} \\
& \leqslant C^{\prime}\left\|v_{1}(t)\right\|_{H_{p}^{1}\left(\mathrm{Q}^{m}\right)}^{p}\left\|v_{2}(t)\right\|_{B C^{1}\left(\mathrm{Q}^{m}\right)^{\prime}}^{p}
\end{aligned}
$$

Combining these discussions yields

$$
\begin{aligned}
\left\|v_{1} v_{2}\right\|_{L_{p}\left(J ; W_{p}^{2-1 / p}\left(\mathrm{Q}^{m}\right)\right)} \leqslant & C^{\prime}\left(\left\|v_{1}\right\|_{B C\left(J \times \mathrm{Q}^{m}\right)}+\left\|v_{1}\right\|_{L_{p}\left(J ; W_{p}^{2-1 / p}\left(\mathrm{Q}^{m}\right)\right)}\right) \\
& \left(\left\|v_{2}\right\|_{C\left(J ; B C^{1}\left(\mathrm{Q}^{m}\right)\right)}+\left\|v_{2}\right\|_{L_{p}\left(J ; W_{p}^{2-1 / p}\left(\mathrm{Q}^{m}\right)\right)}\right) .
\end{aligned}
$$

Using the fact that the embedding constant of ${ }_{0} \mathbb{F}_{2}\left(J, \mathrm{Q}^{m}\right) \hookrightarrow C\left(J ; B C^{1}\left(\mathrm{Q}^{m}\right)\right)$ is independent of $T$ yields the asserted result.

(b) is an immediate consequence of (a) and the fact that the embedding constant of ${ }_{0} \mathbb{F}_{2}\left(J, Q^{m}\right) \hookrightarrow$ $C\left(J ; B C\left(\mathrm{Q}^{m}\right)\right)$ is independent of $T$. 
(c) Suppose that $\left(v_{1}, v_{2}\right) \in \mathbb{F}_{3}\left(J, \mathrm{Q}^{m}\right) \times{ }_{0} \mathbb{F}_{2}\left(J, \mathrm{Q}^{m}\right)$. Then

$$
\begin{aligned}
\left\|v_{1} v_{2}\right\|_{W_{p}^{1 / 2-1 / 2 p}\left(J ; L_{p}\left(\mathrm{Q}^{m}\right)\right)} & \leqslant\left\|v_{1}\right\|_{W_{p}^{1 / 2-1 / 2 p}\left(J ; L_{p}\left(\mathrm{Q}^{m}\right)\right)}\left\|v_{2}\right\|_{B C\left(J \times \mathrm{Q}^{m}\right)} \\
+ & \left(\int_{J} \int_{J}\left\|v_{1}(s)\left(v_{2}(t)-v_{2}(s)\right)\right\|_{L_{p}\left(\mathrm{Q}^{m}\right)}^{p} \frac{1}{|t-s|^{1 / 2+p / 2}} d t d s\right)^{1 / p} .
\end{aligned}
$$

By [55, Lemma 2.5], the comments below [55, formula (3.5)], an analogue of [58, diagram (6.12)] with $B U C$ replaced by $C^{s}$, we obtain the embedding result

$$
{ }_{0} \mathbb{F}_{2}\left(J, \mathrm{Q}^{m}\right) \hookrightarrow C^{s}\left(J ; B C\left(\mathrm{Q}^{m}\right)\right)
$$

for some $s>1 / 2-1 / 2 p$ with an embedding constant $M=M(s)$ uniform in $T$. Thus, we infer that

$$
\begin{aligned}
\int_{J} \int_{J} \| v_{1}(s)\left(v_{2}(t)\right. & \left.-v_{2}(s)\right) \|_{L_{p}\left(\mathrm{Q}^{m}\right)}^{p} \frac{1}{|t-s|^{1 / 2+p / 2}} d t d s \\
& \leqslant C \int_{J}\left\|v_{1}(s)\right\|_{L_{p}\left(\mathrm{Q}^{m}\right)}^{p} \int_{J} \frac{1}{|t-s|^{1 / 2+p / 2-s p}} d t d s\left\|v_{2}\right\|_{C^{s}\left(J ; B C\left(\mathrm{Q}^{m}\right)\right)}^{p} \\
& \leqslant C^{\prime}\left\|v_{1}(s)\right\|_{L_{p}\left(J ; L_{p}\left(\mathrm{Q}^{m}\right)\right)}^{p}\left\|v_{2}\right\|_{C^{s}\left(J ; B C\left(\mathrm{Q}^{m}\right)\right)}^{p} .
\end{aligned}
$$

By an analogous estimate as in (a), see in particular (4.21), one obtains

$$
\left\|v_{1} v_{2}\right\|_{L_{p}\left(J ; W_{p}^{1-1 / p}\left(\mathrm{Q}^{m}\right)\right)} \leqslant C^{\prime}\left\|v_{1}\right\|_{L_{p}\left(J ; W_{p}^{1-1 / p}\left(\mathrm{Q}^{m}\right)\right)}\left\|v_{2}\right\|_{C\left(J ; B C^{1}\left(\mathrm{Q}^{m}\right)\right)} .
$$

Note that the multiplication constant $C_{0}$ in (b) blows up as $T \rightarrow 0$ if ${ }_{0} \mathbb{F}_{j}(J)$ is replaced by $\mathbb{F}_{j}(J)$.

We write

$$
\begin{aligned}
\mathbb{K}_{2}(\hat{z}) w= & \hat{l}_{1} u-\llbracket \psi^{\prime}(\hat{\vartheta}) \rrbracket u+\left(\gamma(\hat{\vartheta})-\hat{\gamma}_{1}\right) \beta(\hat{h}) \partial_{t} \rho+\hat{\gamma}_{1}\left(\beta(\hat{h})-\hat{\beta}_{0}\right) \partial_{t} \rho \\
& -\sigma\left(\mathcal{H}^{\prime}(\hat{h})-\mathcal{H}^{\prime}\left(\hat{h}_{0}\right)\right) \rho+\left(\gamma(\hat{\vartheta}) \beta^{\prime}(\hat{h}) \partial_{t} \hat{h}-\hat{\gamma}_{1} \beta^{\prime}\left(\hat{h}_{0}\right) e^{\Delta_{\Sigma} t}\left(\partial_{t} \hat{h}(0)\right)\right) \rho \\
& +\left(\gamma^{\prime}(\hat{\vartheta}) \beta(\hat{h}) \partial_{t} \hat{h}-\gamma^{\prime}\left(e^{\Delta_{\Sigma} t} \hat{\vartheta}_{0}\right) \hat{\beta}_{0} e^{\Delta_{\Sigma} t}\left(\partial_{t} \hat{h}(0)\right)\right) u .
\end{aligned}
$$

Let $\varepsilon$ sufficiently small be fixed. Recall that

$$
\hat{l}_{1} u-\llbracket \psi^{\prime}(\hat{\vartheta}) \rrbracket u=\left(\llbracket \psi^{\prime}\left(e^{\Delta_{\Sigma} t} \hat{\vartheta}_{0}\right)-\psi^{\prime}(\hat{\vartheta}) \rrbracket\right) u .
$$

Due to fact that $\llbracket \psi^{\prime}\left(e^{\Delta_{\Sigma} t} \hat{\vartheta}_{0}\right)-\psi^{\prime}(\hat{\vartheta}) \rrbracket \in{ }_{0} \mathbb{F}_{2}(J)$ and Lemma 4.3(b), by making $T$ small enough, we can achieve that

$$
\left\|\hat{l}_{1} u-\llbracket \psi^{\prime}(\hat{\vartheta}) \rrbracket u\right\|_{\mathbb{F}_{2}(J)} \leqslant \varepsilon\|u\|_{\mathbb{F}_{2}(J)} .
$$

The arguments for the remaining terms in $\mathbb{K}_{2}(\hat{z}) w$ are similar. Thus given any $\varepsilon>0$, for $T$ small enough, we have

$$
\left\|\mathbb{K}_{2}(\hat{z}) w\right\|_{\mathbb{F}_{2}(J)} \leqslant \varepsilon\|w\|_{0} \mathbb{E}(J)
$$


One can obtain an analogous assertion for $\left\|\mathbb{K}_{3}(\hat{z})\right\|_{\mathcal{L}\left({ }_{0} \mathbb{E}(J),_{0} \mathbb{F}_{3}(J)\right)}$. Indeed,

$$
\left\|\llbracket\left(d(\vartheta)-\hat{d}_{0}\right) \partial_{\nu} u \rrbracket\right\|_{\mathbb{F}_{3}(J)} \leqslant C_{0}\left\|\left(d(\vartheta)-\hat{d}_{0}\right)\right\|_{0_{\mathbb{F}_{3}(J)}}\left\|\llbracket \partial_{\nu} u \rrbracket\right\|_{0} \mathbb{F}_{3}(J) .
$$

Similar estimates also apply to

$$
\left\|\left(\hat{l}_{0}-l(\hat{\vartheta})\right) \partial_{t} \rho\right\|_{\mathbb{F}_{3}(J)},\left\|\left(\llbracket d(\hat{h}) \nabla u \rrbracket \mid M_{4}(\hat{h}) \nabla \hat{h}\right)-\left(\llbracket \hat{d}_{0} \nabla u \rrbracket \mid M_{4}\left(\hat{h}_{0}\right) \nabla \hat{h}_{0}\right)\right\|_{\mathbb{F}_{3}(J)} .
$$

It follows from Lemma 4.3(c) that

$$
\left\|\llbracket d^{\prime}(\hat{\vartheta}) \partial_{\nu} \hat{\vartheta} \rrbracket u\right\|_{\mathbb{F}_{3}(J)} \leqslant C_{0}\left\|\llbracket d^{\prime}(\hat{\vartheta}) \partial_{\nu} \hat{\vartheta} \rrbracket\right\|_{\mathbb{F}_{3}(J)}\|u\|_{\mathbb{F}(J)} .
$$

The remaining terms in $\mathbb{K}_{3}(\hat{z}) w$ can be estimates in an analogous way.

For $\left\|\mathbb{K}_{1}(\hat{z})\right\|_{\mathcal{L}\left({ }_{0} \mathbb{E}(J), \mathbb{F}_{1}(J)\right)}$, one verifies by direct computation that

$$
\begin{aligned}
\left\|\mathbb{K}_{1}(\hat{z}) w\right\|_{\mathbb{F}_{1}(J)} & =\left\|\hat{F}^{\prime}(\hat{z}) w+\hat{d}_{0} M_{2}\left(\hat{h}_{0}\right): \nabla^{2} u\right\|_{\mathbb{F}_{1}(J)} \\
& \leqslant C_{2}\|w\|_{\mathbb{E}(J)}+C_{3}\|w\|_{\mathbb{C}_{1}(J) \times \mathbb{C}_{2}(J) .}
\end{aligned}
$$

Here the constants $C_{2}$ and $C_{3}$ tend to zero as $T \rightarrow 0$.

Proposition 4.4 Let $p>m+3, \sigma>0$. Suppose that $d_{i} \in C^{2}(0, \infty), \gamma, \psi_{i} \in C^{3}(0, \infty)$. Then there exists some constant $\tau_{0}$ such that given any $T \leqslant \tau_{0}$, on $J=[0, T]$, we have

$$
\left.\left\|\mathbb{L}^{-1}\left(\hat{\vartheta}_{0}\right)\right\|_{\mathcal{L}_{0} \mathbb{F}(J), 0} \mathbb{E}(J)\right)\left\|\mathbb{K}^{\prime}\left(\hat{z}_{0}\right)\right\|_{\mathcal{L}\left({ }_{0} \mathbb{E}(J),{ }_{0} \mathbb{F}(J)\right)} \leqslant 1 / 2 .
$$

It follows from a Neuman series argument that

$$
D_{1} \Phi(\bar{z}, 0) \in \operatorname{Lis}\left({ }_{0} \mathbb{E}(J),{ }_{0} \mathbb{F}(J)\right) .
$$

Employing now the implicit function theorem, we attain

$$
\left[(\lambda, \mu, \eta) \mapsto \bar{z}_{\lambda, \mu, \eta}\right] \in C^{k}\left(\mathbb{B}\left(0, r_{0}\right),{ }_{0} \mathbb{E}(J)\right) .
$$

It follows then from Theorem 3.12 that

$$
\bar{z} \in C^{k}\left(\stackrel{0}{J} \times \mathrm{T}_{a / 3} \backslash \Sigma\right) \times C^{k}(\stackrel{\circ}{J} \times \Sigma) .
$$

Remark 4.5. By a similar argument to the proof given at the end of Section 4.2, we can show that

$$
\bar{z} \in C^{k}(\stackrel{\circ}{J} \times \Omega \backslash \Sigma) \times C^{k}(\stackrel{\circ}{J} \times \Sigma) .
$$

Together with (4.18), we thus have

$$
\hat{z} \in C^{k}(\stackrel{\circ}{J} \times \Omega \backslash \Sigma) \times C^{k}(\stackrel{\circ}{J} \times \Sigma) .
$$

For $k \in \mathbb{N} \cup\{\infty\}$, since the Hanzawa transformation is $C^{\infty}$-smooth, the above assertion implies that the solution $(\theta, \Gamma)$ to (1.4) has the same regularity.

But when $k=\omega$, analyticity of the temperature $\theta$, in general, cannot be attained by applying the Hanzawa transformation. 


\section{REFERENCES}

1. Almgren, F. \& L. WAng, L., Mathematical existence of crystal growth with Gibbs-Thomson curvature effects. J. Geom. Anal. 10 (2000), 1-100. Zb10981. 74041 MR1758583

2. Alt, H.W. \& CAFFARELli, L.A., Existence and regularity for a minimum problem with free boundary. J. Reine Angew. Math. 325 (1981), 105-144. Zb10449.35105 MR0618549

3. Alt, H.W. , CAfFarelli, L.A. \& Friedman, A., Variational problems with two phases and their free boundaries. Trans. Amer. Math. Soc. 282 (1984), 431-461. Zbl0844. 35137 MR0732100

4. Amann, H., Anisotropic function spaces and maximal regularity for parabolic problems. Part 1. Function spaces. Jindřich Nečas Center for Mathematical Modeling Lecture Notes, 6. Matfyzpress, Prague, 2009. Zbl1216.35001 MR2907677

5. Amann, H., Function spaces on singular manifolds. Math. Nachr. 286 (2013), 436-475. Zb11280. 46022 MR3048125

6. Amann, H., Anisotropic function spaces on singular manifolds. arXiv.1204.0606.

7. Anderson, D. M. , Cermelli, P., Fried, E., Gurtin, M.E. \& McFadden, G. B., General dynamical sharp-interface conditions for phase transformations in viscous heat-conducting fluids. J. Fluid Mech. 581 (2007), 323-370. Zbl1119.76066 MR2333066

8. Angenent, S. B., Analyticity of the interface of the porous media equation after the waiting time. Proc. Amer. Math. Soc. 102 (1988), 329-336. Zbl0653. 35040 MR0920995

9. Angenent, S. B., Parabolic equations for curves on surfaces. I. Curves with p-integrable curvature, Ann. of Math. (2) 132 (1990), 451-483 . Zbl0789.58070 MR1078266

10. Athanasopoulos, I., Caffarelli, L. \& Salsa, S., Regularity of the free boundary in parabolic phase-transition problems. Acta Math. (2) 176, 245-282 (1996). Zbl0891. 35164 MR1397563

11. Athanasopoulos, I., Caffarelli, L. \& Salsa, S., Phase transition problems of parabolic type: flat free boundaries are smooth. Comm. Pure Appl. Math 51 (1998), 77-112. Zb10924. 35197 MR1486632

12. Berestycki, H., CAfFARElli, L. A. \& NirenberG, L., Uniform estimates for regularization of free boundary problems. Analysis and partial differential equations, 567-619, Lecture Notes in Pure and Appl. Math., 122, Dekker, New York, 1990. Zbl0702.35252 MR1044809

13. Browder, F. E., Analyticity and partial differential equations I. Amer. J. Math. 84 (1962), 666-710. Zb10124.31004 MR0150463

14. Caffarelli, L. A., The regularity of free boundaries in higher dimensions. Acta Math. 139 (1977), 155-184. Zbl0386.35046 MR0454350

15. Caffarelli, L. A., Some aspects of the one-phase Stefan problem. Indiana Univ. Math. J. 27 (1978), 73-77. Zbl0393.35064 MR0466965

16. CAfFARElli, L. A., A Harnack inequality approach to the regularity of free boundaries. I. Lipschitz free boundaries are $C^{1+\alpha}$. Rev. Mat. Iberoamericana 3 (1987), 139-162 . Zb10676. 35085 MR0990856

17. Caffarelli, L. A. \& Evans, L. C., Continuity of the temperature in the two-phase Stefan problem. Arch. Rational Mech. Anal. 81 (1983), 199-220. Zb10516.35080 MR0683353

18. Caffarelli, L. A. \& Friedman, A., Continuity of the temperature in the Stefan problem. Indiana Univ. Math. J. 28, (1979), 53-70. Zbl0406. 35032 MR0523623

19. Caginalp, G., An analysis of a phase field model of a free boundary. Arch. Rational Mech. Anal. 92 (1986), 205-245. Zbl0608. 35080 MR0816623

20. Chalmers, B., Principles of Solidification. Krieger, Huntington, 1977.

21. Denk, R., Hieber, M. \& PRÜss, J., R-boundedness, Fourier multipliers and problems of elliptic and parabolic type. Mem. Amer. Math. Soc. 166, (2003). Zbl1274. 35002 MR2006641

22. DenK, R., PrüSS, J. \& ZACHER, R., Maximal Lp-regularity of parabolic problems with boundary dynamics of relaxation type. J. Funct. Anal. 255 (2008), 3149-3187. Zb11160. 35030 MR2464573 
23. DiBenedetto, E., Regularity properties of the solution of an $n$-dimensional two-phase Stefan problem. Boll. Un. Mat. Ital. Suppl. (1980), 129-152. Zb10458.35098 MR0677695

24. DiBenedetto, E., Continuity of weak solutions to certain singular parabolic equations. Ann. Mat. Pura Appl. 130 (1982), 131-176. Zb10503.35018 MR0663969

25. Duvaut, G., Résolution d'un probléme de Stefan (fusion d'un bloc de glace á zéro degré). C. R. Acad. Sci. Paris Sér. A-B 276 (1973), A1461-A1463. Zb10258. 35037 MR0328346

26. Escher, J., PRÜSS, J. \& SimOnett, G., A new approach to the regularity of solutions for parabolic equations. Evolution equations, 167-190, Lecture Notes in Pure and Appl. Math. 234, Dekker, New York, 2003. Zbl1070.35009 MR2073744

27. Escher, J., Prüss, J. \& Simonett, G., Analytic solutions for a Stefan problem with Gibbs-Thomson correction. J. Reine Angew. Math. 563 (2003), 1-52. Zbl1242.35220 MR2009238

28. Escher, J. \& Simonett, G., Analyticity of the interface in a free boundary problem. Math. Ann. 305 (1996), 439-459. Zbl0857.76086 MR1397432

29. Escher, J. \& Simonett, G., A center manifold analysis for the Mullins-Sekerka Model. J. Differential Equations 143 (1998), 267-292. Zbl0896 . 35142 MR1607952

30. Friedman, A., The Stefan problem in several space variables. Trans. Amer. Math. Soc. 133 (1968), 51-87. Zb10162.41903 MR0227625

31. Friedman, A., The Stefan problem with small surface tension. Trans. Amer. Math. Soc. 328 (1991), 465-515. Zb10744.35068 MR1040260

32. Friedman, A. \& Kinderlehrer, D., A one phase Stefan problem. Indiana Univ. Math. J. 24 (1974/75), 1005-1035 Zbl0334.49002 MR0385326

33. Friedman, A. \& Reitich, F., Variational principles and free-boundary problems. Second edition. Robert E. Krieger Publishing Co., Inc., Malabar, FL 1988. MR1009785

34. Gurtin, M.E., On the two-phase Stefan problem with interfacial energy and entropy. Arch. Rational Mech. Anal. 96, no. 3, 199-241 (1986). Zbl0654.73008 MR0855304

35. Gurtin, M.E., Multiphase thermomechanics with interfacial structure. I. Heat conduction and the capillary balance law. Arch. Rational Mech. Anal. 104 (1988), 195-221. Zbl0723. 73016 MR1017288

36. Gurtin, M. E., Toward a nonequilibrium thermodynamics of two-phase materials. Arch. Rational Mech. Anal. 100 (1988), 275-312. Zbl0673.73007 MR0918798

37. HADŽIĆ, M., Orthogonality conditions and asymptotic stability in the Stefan problem with surface tension. Arch. Ration. Mech. Anal. 203 (2012), 719-745. Zbl1268.80006 MR2928131

38. HADŽIĆ, M. \& Guo, Y., Stability in the Stefan problem with surface tension (I). Comm. Partial Differential Equations 35 (2010), 201-244. Zb11195. 35303 MR2748623

39. Hanzawa, E. I., Classical solution of the Stefan problem. Tôhoku Math. J (2) 33 (1981), 297-335. Zb10571.35109 MR0633045

40. Hartman, P., Crystal Growth: An Introduction. North-Holland, 1973.

41. Ishi, M. \& TAKASHI, H., Thermo-fluid dynamics of two-phase flow. Springer, New York, 2006. Zbl1204.76002

42. Kamenomostskaja, S. L., On Stefan's problem. Mat. Sb. (N.S.) 53 (95) 489-514 (1961). Mr0141895

43. Kinderlehrer, D. \& Nirenberg, L., Regularity in free boundary problems. Ann. Scuola Norm. Sup. Pisa Cl. Sci. (4) 4 (1977), 373-391. Zbl0352 . 35023 MR0440187

44. Kinderlehrer, D. \& Nirenberg, L., The smoothness of the free boundary in the one phase Stefan problem. Comm. Pure Appl. Math. 31 (1978), 257-282. Zb10391. 35060 Mr0480348

45. Kinderlehrer, D., Nirenberg, L. \& Spruck, J., Regularity in elliptic free boundary problems. $J$. Analyse Math. 34 (1978), 86-119 (1979). Zb10402. 35045 MR0531272 
46. Kinderlehrer, D. \& Stampacchia, G., An introduction to variational inequalities and their applications. Pure and Applied Mathematics, 88. Academic Press, Inc. [Harcourt Brace Jovanovich, Publishers], New York-London, 1980. Zbl0457. 35001 MR0567696

47. KосH, H., Classical solutions to phase transition problems are smooth. Comm. Partial Differential Equations 23 (1998), 389-437. Zbl0904. 35014 MR1620648

48. LANGer, J. S., Instabilities and pattern formation of crystal growth. Rev. Mod. Phys. 52 (1980), 1-28.

49. LuCKhaus, S., Solutions for the two-phase Stefan problem with the Gibbs-Thomson law for the melting temperature. European J. Appl. Math. 1 (1990), 101-111. Zb10734. 35159 MR1117346

50. Lunardi, A., Analytic Semigroups and Optimal Regularity in Parabolic Problems. Birkhäuser Verlag, Basel, 1995. Zbl0816. 35001 MR1329547

51. Matano, H., Asymptotic behavior of the free boundaries arising in one phase Stefan problems in multi-dimensional spaces. Lecture Notes in Num. Appl. Anal. 5, Kinokuniya, Tokyo, 133-151 (1982). Zb10569.35076 MR0730240

52. MeǏrmanov, A. M., On the classical solution of the multidimensional Stefan problem for quasilinear parabolic equations. Mat. Sb. (N.S.) 112(154) (1980), 170-192. MR0585773

53. MeǏrmanov, A. M., The Stefan problem. Translated from the Russian by Marek Niezgódka and Anna Crowley. With an appendix by the author and I. G. Götz. de Gruyter Expositions in Mathematics, 3. Walter de Gruyter \& Co., Berlin, 1992. Zb10751.35052 MR1154310

54. MeǏrmanov, A.M., The Stefan problem with surface tension in the three-dimensional case with spherical symmetry: nonexistence of the classical solution. European J. Appl. Math. 5 (1994), 1-19. Zb10812.35165 MR1270785

55. Meyries, M. \& Schnaubelt, R., Interpolation, embeddings and traces of anisotropic fractional Sobolev spaces with temporal weights. J. Funct. Anal. 262 (2012), 1200-1229. Zbl1250.46022 MR2863860

56. Mullins, W. W. \& SeKerka, R. F., Morphological stability of a particle growing by diffusion or heat flow. J. Appl. Phys. 34 (1963), 323-329.

57. Mullins, W. W. \& SeKerka, R. F., Stability of a planar interface during solidification of a dilute binary alloy J. Appl. Phys. 35 (1964), 444-451.

58. PrÜSS, J., SAAL, J. \& Simonett, G., Existence of analytic solutions for the classical Stefan problem. Math. Ann. 338 (2007), 703-755. Zbl1130.35136 MR2317935

59. PrÜss, J., SAAL, J. \& Simonett, G., Stability of equilibria for the Stefan problem with surface tension. SIAM J. Math. Anal. 40 (2008), 675-698. Zbl1157. 35502 MR2438781

60. PRÜSS, J. \& SImonett, G., Analytic solutions for the two-phase Navier-Stokes equations with surface tension and gravity. Parabolic problems. Progr. Nonlinear Differential Equations Appl., 80, Birkhäuser/Springer Basel AG, Basel, 2011. Zb11247. 35207 MR3052594

61. PRÜss, J. \& Simonett, G., On the two-phase Navier-Stokes equations with surface tension. Interfaces Free Bound. 12 (2010), 311-345. Zbl1202 . 35359 MR2727674

62. PrüsS, J. \& Simonett, G., On the Rayleigh-Taylor instability for the two-phase Navier-Stokes equations. Indiana Univ. Math. J. 59 (2010), 1853-1871. Zbl1234. 35323 MR2919738

63. Prüss, J. \& Simonett, G., On the manifold of closed hypersurfaces in $\mathbb{R}^{n}$. Discrete Contin. Dyn. Syst. 33 (2013), 5407-5428. Zbl1274. 35434 MR3297714

64. PrÜss, J. \& Simonett, G., Moving interfaces and quasilinear parabolic evolution equations. Monographs in Mathematics, Birkhäuser Verlag. To appear.

65. PrÜSS, J., Simonett, G. \& ZACher, R., Qualitative behavior of solutions for thermodynamically consistent Stefan problems with surface tension. Arch. Ration. Mech. Anal. 207 (2013), 611-667. Zb11269.80004 MR3005325 
66. RADKEVICH, E. V., The Gibbs-Thompson correction and conditions for the existence of a classical solution of the modified Stefan problem. Dokl. Akad. Nauk SSSR316 6, 1311-1315; translation in Soviet Math. Dokl. 43 (1991), 274-278. Zb10782.35087 MR1122261

67. Rodrigues, J.-F., The variational inequality approach to the one-phase Stefan problem. Acta Appl. Math. 8 (1987), 1-35. Zbl0653. 35083 MR0871691

68. Rodrigues, J.-F., The Stefan problem revisited. In Mathematical models for phase change problems, volume 88 of Internat. Ser. Numer. Math., 129-190. Birkhäuser, Basel, 1989. Zb10699. 35265 MR1038069

69. Rodrigues, J.-F., Variational methods in the Stefan problem. In Phase transitions and hysteresis (Montecatini Terme, 1993), volume 1584 of Lecture Notes in Math., 147-212. Springer, Berlin, 1994. Zb10819.35154 MR1321833

70. RÖGER, M., Solutions for the Stefan problem with Gibbs-Thomson law by a local minimisation. Interfaces Free Bound. 6 (2004), 105-133. Zbl1050.35155 MR2047075

71. Rubinstein, L. I., The Stefan Problem. Translations of Mathematical Monographs, Vol. 27, American Mathematical Society, Providence, R.I. 1971. MR0351348

72. ShaO, Y., Real analytic solutions to the Willmore Flow. Ninth MSU-UAB Conference on Differential Equations and Computational Simulations. Electron. J. Diff. Eqns., Conf. 20 (2013), 151-164. Zbl1295. 35160 MR3128075

73. ShaO, Y., A family of parameter-dependent diffeomorphisms acting on function spaces over a Riemannian manifold and applications to geometric flows. NoDEA Nonlinear Differential Equations Appl. 22 (2015), 45-85. Zbl1322.58016 MR3311893

74. Shao, Y. \& Simonett, G., Continuous maximal regularity on uniformly regular Riemannian manifolds. J. Evol. Equ. 1 (2014), 211-248. Zb11295.35161 MR3169036

75. Triebel, H., Interpolation Theory, Function Spaces, Differential Operators. North-Holland Publishing Co., Amsterdam-New York, 1978. Zb10387.46033 MR0503903

76. Visintin, A., Remarks on the Stefan problem with surface tension. Boundary value problems for partial differential equations and applications, 455-460, RMA Res. Notes Appl. Math., 29, Masson, Paris (1993). Zb10803.35173 MR1260478

77. Visintin, A., Models of phase transitions. Progress in Nonlinear Differential Equations and their Applications. 28. Birkhäuser Boston, Inc., Boston, MA, 1996. Zb10882. 35004 MR1423808

78. ZIEMER, W. P., Interior and boundary continuity of weak solutions of degenerate parabolic equations. Trans. Amer. Math. Soc. 271 (1982), 733-748. Zb10506. 35053 MR0654859 